Portland State University

PDXScholar

$1-1-2010$

\title{
Stochastic Orders in Heterogeneous Samples with Applications
}

Maochao Xu

Portland State University

Follow this and additional works at: https://pdxscholar.library.pdx.edu/open_access_etds

Part of the Mathematics Commons

Let us know how access to this document benefits you.

Recommended Citation

$\mathrm{Xu}$, Maochao, "Stochastic Orders in Heterogeneous Samples with Applications" (2010). Dissertations and Theses. Paper 391.

https://doi.org/10.15760/etd.391

This Dissertation is brought to you for free and open access. It has been accepted for inclusion in Dissertations and Theses by an authorized administrator of PDXScholar. Please contact us if we can make this document more accessible: pdxscholar@pdx.edu. 
Stochastic Orders in Heterogeneous Samples with Applications

by

Maochao Xu

A dissertation submitted in partial fulfilment of the requirements for the degree of

\author{
Doctor of Philosophy \\ in \\ Mathematical Sciences
}

Dissertation Committee:

Subhash Kochar, Chair

Robert Fountain

Jong Sung Kim

Kuan-Pin Lin

Michael Perlman

Erik Bodegom

Portland State University

(c) 2010 


\begin{abstract}
The statistics literature has mostly focused on the case when the data available is in the form of a random sample. In many cases, the observations are not identically distributed. Such samples are called heterogeneous samples. The study of heterogeneous samples is of great interest in many areas, such as statistics, econometrics, reliability engineering, operation research and risk analysis.

Stochastic orders between probability distributions is a widely studied concept. There are several kinds of stochastic orders that are used to compare different aspects of probability distributions like location, variability, skewness, dependence, etc.

In this dissertation, most of the work is devoted to investigating the properties of statistics based on heterogeneous samples with the aid of stochastic orders. We will see the effect of the change in the stochastic properties of various functions of observations as their parameters change. The theory of majorization will be used for this purpose.

First, order statistics from heterogeneous samples will be investigated. Order statistics appear everywhere in statistics and related areas. The $k$-out-of- $n$ systems are building blocks of a coherent system. The lifetime of such a system is the same as that of the $(n-k+1)$ th order statistic in a sample size of $n$. Stochastic comparisons between order statistics have been studied extensively in the literature in case the parent observations are independent and identically distributed.
\end{abstract}


However, in practice this assumption is often violated as different components in a system may not have the same distribution. Comparatively less work has been done in the case of heterogeneous random variables, mainly because of the reason that their distribution theory is very complicated. Some open problems in the literature have been solved in the dissertation. Some new problems associated with order statistics have been investigated in the thesis.

Next, stochastic properties of spacings based on heterogeneous observations are studied. Spacings are of great interest in many areas of statistics, in particular, in the characterizations of distributions, goodness-of-fit tests, life testing and reliability models. In particular, the stochastic properties of the sample range are investigated in detail. Applications in reliability theory are highlighted.

The relative dependence between extreme order statistics will be investigated in Chapter 4. In particular, the open problem discussed in Dolati, et al. [18] is solved in this Chapter.

In the last Chapter, convolutions of random variables from heterogeneous samples will be investigated. Convolutions have been widely used in many areas to model many practical situations. For example, in reliability theory, it arises as the lifetime of a redundant standby system; in queuing theory, it is used to model the total service time by an agent in a system; in insurance, it is used to model total claims on a number of policies in the individual risk model. I will compare the dispersion and skewness properties of convolutions of different heterogeneous 
samples. The tail behavior of convolutions are investigated as well.

The work in this dissertation has significant applications in many diverse areas of applied probability and statistics. For example, statistics based on order statistics and spacings from heterogeneous samples arise in studying the robust properties of statistical procedures; the work on order statistics will also provide a better estimation of lifetime of a coherent system in reliability engineering; convolution results will be of great interest in insurance and actuarial science for evaluating risks. 
In memory of my mother 


\section{Acknowledgements}

I would like to express the deepest appreciation to my supervisor, Professor Subhash C. Kochar, whose supervision and encouragement enabled me to develop a deep understanding of the subject. I am also grateful for his kindness and support during my study, which helped me in overcoming difficulties in my life. Without his guidance and persistent help, this dissertation would not have been possible.

I deeply thank my allied area advisor, Professor Kuan-Pin Lin, who introduced me to the interesting area of econometrics. His enthusiasm and encouragement enabled me to work in a different research area, which is of great benefit to me in the academic career.

I would like to thank my committee members, Professor Robert Fountain, Professor Jong Sung Kim and Professor Erik Bodegom. I am grateful to Professor Michael Perlman of the University of Washington for his agreeing to act as an external member on my committee.

I express my gratitude to our Department for arranging a TA position for me which enabled me to study at Portland State University. I am grateful for the financial support from Eugene Enneking Doctoral Fellowship, which enabled me to be free from teaching, and to concentrate on the thesis. My thanks also go to faculty and the office staff for their continuous help.

I thank Professor Xiaohu Li, who introduced me to the area of stochastic order. I also would like to thank my friends, Peng Zhao, Xiaoxiao Hu, Zhouping Li and 
Yashi Wang for some academic discussion and personal help during my study.

Finally, my most heartfelt thanks to my wife Yan Jin, and my mother in law who took care of our son for one year. 


\section{Contents}

$\begin{array}{ll}\text { Abstract } & \text { i }\end{array}$

Dedication

Acknowledgements $\quad$ v

$\begin{array}{ll}\text { List of Tables } & \mathbf{x}\end{array}$

List of Figures $\quad x i$

1 Introduction 1

1.1 Introduction . . . . . . . . . . . . . . . 1

1.1.1 Stochastic orders ............... 3

1.1.1.1 Magnitude orders . . . . . . . . . . 3

1.1.1.2 Dispersion orders . . . . . . . . . 7

1.1.1.3 Shape orders . . . . . . . . . . 8

1.1.2 Majorization orders . . . . . . . . . . . 11

1.1.3 Dependence orders . . . . . . . . . . . . . . 14 
2.1 Introduction . . . . . . . . . . . . . . . 18

2.2 Stochastic comparisons of parallel systems by likelihood ratio order 24

2.3 Comparisons of parallel systems according to convex transform

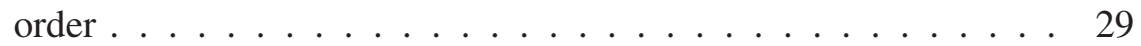

2.3 .1 Introduction . . . . . . . . . . . . . . 29

2.3.2 Which parallel system ages faster? . . . . . . . . . 31

2.3.3 Dispersion ................... 37

2.4 Starorder . . . . . . . . . . . . . . . . . . . 42

2.4 .1 PHR models . . . . . . . . . . . . . . . 46

2.5 Concluding remarks . . . . . . . . . . . . . . . . . 48

3 Spacings $\quad 50$

3.1 Introduction . . . . . . . . . . . . . . 50

3.2 Equivalent characterizations . . . . . . . . . . . 52

3.3 Range . . . . . . . . . . . . . . . . . 58

3.3.1 Reversed hazard rate order . . . . . . . . . . . . 60

3.3.2 Likelihood ratio order $\ldots \ldots \ldots 6$

3.3.3 Dispersive order ............... 71

3.4 Application . . . . . . . . . . . . . . 75

3.5 Concluding remarks . . . . . . . . . . . . . . . 77 
4 Dependence between extreme order statistics 79

4.1 Introduction . . . . . . . . . . . . . . . . . . 79

4.2 SI dependence order $\ldots \ldots \ldots$. . . . . . . . . . . . 82

5 Stochastic comparisons of convolutions of independent random vari-

$\begin{array}{ll}\text { ables } & 84\end{array}$

5.1 Introduction . . . . . . . . . . . . . . 84

5.2 Right spread order . . . . . . . . . . . . . . . 86

5.3 Tail behavior of the convolutions of gamma random variables . . . 99

5.3.1 Introduction . . . . . . . . . . . . . . . 99

5.3.2 Star ordering between convolutions of gamma random vari-

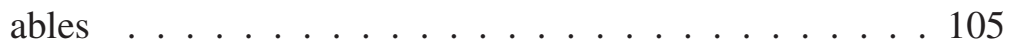

5.3.3 Right spread order . . . . . . . . . . . . . . . . 114

5.4 Applications . . . . . . . . . . . . . . . . . 117

5.4 .1 Reliability theory . . . . . . . . . . . . . 117

5.4 .2 Economics ................... . . 119

5.4.3 Actuarial science . . . . . . . . . . . . . . . 119

5.5 Concluding remarks . . . . . . . . . . . . . . . . 121

$\begin{array}{lr}\text { Bibliography } & 123\end{array}$ 


\section{List of Tables}

3.1 Parameters for different orders . . . . . . . . . . . . . 77 


\section{List of Figures}

2.1 The density curves with different parameters. . . . . . . . . . 36

5.1 The density curves with different parameters. . . . . . . . . . . 98

5.2 Expected shortfall of the total claim with different exponential pa-

rameters. . . . . . . . . . . . . . . . 121 


\section{Chapter 1}

\section{Introduction}

\subsection{Introduction}

The Cauchy distribution is a well-known distribution in probability and statistics. In physics, it is known as Lorentz or the Breit-Wigner distribution, as it is described as the solution to the differential equation describing forced resonance. Suppose we want to compare two Cauchy distributions. What is the best way to do that? In general, the simplest way to compare two distribution functions is by their associated means and variances or standard deviations. However, for Cauchy distributions, means and variances do not exist! Moving apart from Cauchy distributions, means and variances are not very informative in comparing distributions. Hence, new and more informative methods for comparing distributions are needed. In many cases, one could express various functional forms of the underlying distributions, such as, quantiles, survival function, hazard rate function and other suitable transforms. Comparisons based on these functional forms of distributions often establish partial orders among them, which are well-known 
as stochastic orders. Stochastic orders have been used during the last decades in many areas of probability and statistics, including reliability theory, queueing theory, survival analysis, biology, economics, insurance, actuarial science, operations research, and management science. One may refer to two excellent books by Shaked and Shanthikumar [60] and Müller and Stoyan [50] on this topic.

Heterogeneous samples arise in many practical situations. For example, in engineering, a complex engineering system is often composed of many different types of electrical components. Investigating the reliability of such a system, heterogeneous samples arise in a natural way, which are the failure times of electrical components collected from experiments. In insurance, a total claim for the portfolio of an insurer may be consisted of different sub-claims, which are from different distributions. In statistical inference, statistics based on heterogeneous samples arise in the study of robust properties of statistical procedures. Accordingly, the topic of heterogenous samples plays an important role in these areas. However, such study is often challenging as the distribution theory of heterogeneous samples is often complicated. For example, assume $X_{1}, X_{2}, \ldots, X_{n}$ are independent exponential random variables with rate parameters $\lambda_{1}, \lambda_{2}, \ldots, \lambda_{n}$, respectively. It is well-known that, if $\lambda_{i}$ 's are equal, then $\sum_{i=1}^{n} X_{i}$ has gamma distribution with shape parameter $n$ and scale parameter $1 / \lambda_{i}$. However, when $\lambda_{i}$ 's are not all equal, the distribution function of $\sum_{i=1}^{n} X_{i}$ is very complicated, and hence the distributional properties of $\sum_{i=1}^{n} X_{i}$ remain unknown. 
In this dissertation, I use the tool of stochastic orders to investigate the stochastic properties of statistics based on heterogeneous samples, like magnitude, dispersion, dependence and skewness. The work presented in the dissertation will not only complement the distribution theory of heterogeneous samples but also have significant applications in many areas of applied probability and statistics. In particular, some open problems in the literature have been solved in the dissertation.

\subsubsection{Stochastic orders}

\subsubsection{Magnitude orders}

Let $X$ and $Y$ be two random variables with distributions $F$ and $G$, and survival functions $\bar{F}=1-F$ and $\bar{G}=1-G$, if $X$ is less likely than $Y$ to take on large values, then, intuitively, the survival function of $X$ will be smaller than the survival function of $Y$ at any fixed point. This leads to the usual stochastic order.

Definition 1.1.1 $X$ is said to be smaller than $Y$ in the usual stochastic order, denoted by $X \leq_{s t} Y$, if $\bar{F}(x) \leq \bar{G}(x)$, or equivalently, $F(x) \geq G(x)$.

It is well-known that for all increasing functions $\phi$,

$$
X \leq_{s t} Y \Longleftrightarrow \mathrm{E}[\phi(X)] \leq \mathrm{E}[\phi(Y)]
$$

provided expectations exist. In economics and utility theory, the usual stochastic order is named as the first-order stochastic dominance. Note that $X$ is stochasti- 
cally smaller than $Y$ if and only if every quantile of $X$ is smaller than the corresponding quantile of $Y$.

Suppose that $X$ and $Y$ are life lengths of two electronic components and satisfy $X \leq_{s t} Y$. If both components are observed to be alive at time $t>0$, one might conjecture that their residual lives would also be stochastically ordered. However, this is not true in general (cf. Müller and Stoyan [50] and Marshall and Olkin [48]). Hence, a stronger concept than usual stochastic order is needed. The following order is motivated by the fact that the above conjecture is false.

Definition 1.1.2 $X$ is said to be smaller than $Y$ in the hazard rate order, denoted by $X \leq_{h r} Y$, if

$$
P(X>x+t \mid X>t) \leq P(Y>x+t \mid Y>t), \quad \text { for all } x \geq 0 \text { and all } t,
$$

or equivalently,

$$
\frac{\bar{G}(t)}{\bar{F}(t)} \text { is increasing in } t \text {. }
$$

Now, suppose that the components $X$ and $Y$ have failed before observation time $t>0$. If $X \leq_{s t} Y$, is it necessary that the 'inactive time' $[t-X \mid X \leq t]$ of $X$ is stochastically larger that the 'inactive time' $[t-Y \mid Y \leq t]$ of $Y$ ? The answer is negative. The following definition is proposed to resolve this question.

Definition 1.1.3 $X$ is said to be smaller than $Y$ in the reversed hazard rate order, denoted by $X \leq_{r h} Y$, if

$$
P(t-X>x \mid X \leq t) \geq P(t-Y>x \mid Y \leq t), \quad \text { for all } x \geq 0 \text { and all } t,
$$


or equivalently,

$$
\frac{G(t)}{F(t)} \text { is increasing in } t \text {. }
$$

It is worth noting that $X \leq_{h r} Y$ is equivalent to

$$
X_{t}=[X-t \mid X>t] \leq_{s t}[Y-t \mid Y>t]=Y_{t}
$$

where $X_{t}$ is termed as the residual life of $X$ at time $t$ in reliability theory and survival analysis. Note that

$$
\mathrm{E}\left(X_{t}\right)=\frac{\int_{t}^{\infty} \bar{F}(x) d x}{\bar{F}(t)}
$$

for $t<t^{*}=\sup \{t: \bar{F}(t)>0\}$.

An interesting order based on the mean residual life is defined below.

Definition 1.1.4 $X$ is said to be smaller than $Y$ in the mean residual life order, denoted by $X \leq_{m r l} Y$, if

$$
\mathrm{E}\left(X_{t}\right) \leq \mathrm{E}\left(Y_{t}\right) \text { for all } t
$$

There are other situations, where one would like to have

$$
[X \mid X \in E] \leq_{s t}[Y \mid Y \in E]
$$

for all possible events $E$. It turns out that this requirement leads to the likelihood ratio order which is quite useful. 
Definition 1.1.5 $X$ is said to be smaller than $Y$ in the likelihood ratio order, denoted by $X \leq_{l r} Y$, if

$$
P(X>x \mid a<X \leq b) \leq P(Y>x \mid a<Y \leq b) \text { for all } x \text { and } a<b .
$$

When the density functions of $X$ and $Y$ exist, $X \leq_{l r} Y$ is equivalent to

$$
f(t) g(s) \leq f(s) g(t) \text { for all } s \leq t,
$$

where $f$ and $g$ are density functions of $X$ and $Y$.

The following chain of implications holds among the above univariate stochastic orders.

$$
\begin{aligned}
& X \leq_{l r} Y \quad \Longrightarrow X \leq_{h r} Y \quad \Longrightarrow \quad X \leq_{m r l} Y \\
& \Downarrow \quad \Downarrow \quad \Downarrow \\
& X \leq_{r h} Y \quad \Longrightarrow \quad X \leq_{s t} Y \quad \Longrightarrow \quad \mathrm{E}(X) \leq \mathrm{E}(Y) \text {. }
\end{aligned}
$$

The usual stochastic order has the following multivariate version.

Definition 1.1.6 The random vector $\mathbf{X}=\left(X_{1}, \ldots, X_{n}\right)$ is said to be smaller than another random vector $\mathbf{Y}=\left(Y_{1}, \ldots, Y_{n}\right)$, denoted by $\mathbf{X} \stackrel{s t}{\preceq} \mathbf{Y}$, according to the multivariate stochastic ordering if

$$
\mathrm{E}[\phi(\mathbf{X})] \leq \mathrm{E}[\phi(\mathbf{Y})]
$$


for all increasing functions $\phi$. It is known that the multivariate stochastic order implies the component-wise stochastic order. For more details on the univariate and multivariate stochastic orders, see Shaked and Shanthikumar [60] and Müller and Stoyan [50].

\subsubsection{Dispersion orders}

A basic concept to compare the spread or variability in two probability distributions is through dispersive order as defined below.

Definition 1.1.7 $X$ is said to be less dispersed than $Y$, denoted by $X \leq_{\text {disp }} Y$, if

$$
F^{-1}(\beta)-F^{-1}(\alpha) \leq G^{-1}(\beta)-G^{-1}(\alpha)
$$

for all $0<\alpha \leq \beta<1$, where $F^{-1}$ and $G^{-1}$ denote their corresponding right continuous inverses.

When the density functions of $X$ and $Y$ exist, $X \leq_{\text {disp }} Y$ if and only if

$$
g\left(G^{-1}(u)\right) \leq f\left(F^{-1}(u)\right) \quad \text { for all } u \in(0,1)
$$

For more discussion, one may refer to Section 3.B of Shaked and Shanthiku$\operatorname{mar}[60]$.

A weaker order called the right spread order has been proposed in FernándezPonce, et al. [20], or excess wealth order in Shaked and Shanthikumar [59] to compare the variabilities of two distributions. 
Definition 1.1.8 $X$ is said to be less right spread than $Y$, denoted by $X \leq_{R S} Y$, if

$$
\int_{F^{-1}(p)}^{\infty} \bar{F}(x) d x \leq \int_{G^{-1}(p)}^{\infty} \bar{G}(x) d x, \quad \text { for all } 0 \leq p \leq 1 .
$$

It is known that

$$
X \leq_{d i s p} Y \Longrightarrow X \leq_{R S} Y \Longrightarrow \operatorname{Var}(X) \leq \operatorname{Var}(Y)
$$

\subsubsection{Shape orders}

MacGillivray and Blanda [46] defined distributions $F$ and $G$ to have the same shape if for some $a$ and $b$,

$$
F(x)=G(a x+b) \text { for all } x .
$$

It is very interesting to compare the shapes of two different distributions. For example, in insurance, one insurance company compares two different risks $X$ and $Y$. If risk $X$ is more negatively skewed than risk $Y$, it means risk $X$ will have a larger tail probability. The company may consider to charge more premium for risk $X$. In the following, we will review some stochastic orders for comparing shapes of distributions. It should be noted that shape orders are preorders but not partial orders, and are scale free.

Definition 1.1.9 $X$ is said to be smaller than $Y$ in the convex transform order, denoted by $X \leq_{c} Y$, if and only if, $G^{-1} F(x)$ is convex in $x$ on the support of $X$, where $G^{-1}$ denotes the right continuous inverse. 
If $X \leq_{c} Y$, then $Y$ is more skewed than $X$ as explained in van Zwet [63] and Marshall and Olkin [48]. The convex transform order is also called more IFR (increasing failure rate) order in reliability theory, since when $f$ and $g$ exist, the convexity of $G^{-1} F(x)$ means that

$$
\frac{f\left(F^{-1}(u)\right)}{g\left(G^{-1}(u)\right)}=\frac{r_{X}\left(F^{-1}(u)\right)}{r_{Y}\left(G^{-1}(u)\right)}
$$

is increasing in $u \in[0,1]$, where $r_{X}$ and $r_{Y}$ mean hazard rate functions of $X$ and $Y$. Thus $X \leq_{c} Y$ can be interpreted to mean that $X$ ages faster than $Y$ in some sense. Note that neither $F$ nor $G$ need to be IFR for this definition to hold. It can be seen that $X$ is an IFR distribution if and only if it is convex ordered with respect to exponential distribution, which has a constant failure rate. It is known that if $X_{\alpha_{i}}$ has Gamma distribution with shape parameter $\alpha_{i}, i=1,2$, then $X_{\alpha_{1}} \leq_{c} X_{\alpha_{2}}$ for $\alpha_{2} \leq \alpha_{1}$.

Replacing the convexity of $G^{-1} F(\cdot)$ by the weaker starshaped property leads to the star order.

Definition 1.1.10 $X$ is said to be smaller than $Y$ in the star order, denoted by $X \leq_{\star} Y$, if and only if, $G^{-1} F(x) / x$ is increasing in $x$ on the support of $X$.

The star order is also called more IFRA (increasing failure rate in average) order in reliability theory, since the average failure of $F$ at $x$ is

$$
\bar{r}_{X}(x)=\frac{1}{x} \int_{-\infty}^{x} r_{X}(u) d u=\frac{-\ln \bar{F}(x)}{x}
$$


Thus $F \leq_{\star} G$ can be interpreted in terms of average failure rates as

$$
\frac{\bar{r}_{X}\left(F^{-1}(u)\right)}{\bar{r}_{Y}\left(G^{-1}(u)\right)}
$$

is increasing in $u \in(0,1] . X$ is an IFRA distribution if and only if $F$ is starordered with respect to exponential distribution.

Kochar and Wiens [38] introduced the following shape order from the point of reliability theory.

Definition 1.1.11 $X$ is said to be more NBUE (new better than used in expectation) than $Y$ or $X$ is smaller than $Y$ in the NBUE order, denoted by $X \leq_{\text {NBUE }} Y$, if

$$
\frac{1}{\mathrm{E}(X)} \int_{F^{-1}(u)}^{\infty} \bar{F}(x) d x \leq \frac{1}{\mathrm{E}(Y)} \int_{G^{-1}(u)}^{\infty} \bar{G}(x) d x, \quad \text { for all } u \in(0,1] .
$$

Note that $X$ is NBUE if and only if $X \leq_{\text {NBUE }} Y$, where $Y$ is an exponential random variable. When $\mathrm{E}(X)=\mathrm{E}(Y)$, it is seen that the order $\leq_{R S}$ is equivalent to the order $\leq_{\text {NBUE }}$. However, they are distinct when $\mathrm{E}(X) \neq \mathrm{E}(Y)$. For more details, please refer to Kochar, Li and Shaked [37].

Lorenz order used in Economics to compare income inequalities, is also a shape order.

Definition 1.1.12 $X$ is said to be smaller than $Y$ in the Lorenz order, denoted by $X \leq_{\text {Lorenz }} Y$, if

$$
\frac{1}{\mathrm{E}(X)} \int_{-\infty}^{F^{-1}(u)} x d F(x) \geq \frac{1}{\mathrm{E}(Y)} \int_{-\infty}^{G^{-1}(u)} x d G(x), \quad \text { for all } u \in(0,1] .
$$


The function

$$
L_{X}(u)=\frac{1}{\mathrm{E}(X)} \int_{-\infty}^{F^{-1}(u)} x d F(x)
$$

is known as lorenz curve in the economics literature. It is often used to express inequality in incomes. It is pointed out in Kochar [33] that the Lorenz orde is equivalent to the HNBUE order in reliability.

We have the following chain of implications

$$
X \leq_{c} Y \Longrightarrow X \leq_{\star} Y \Longrightarrow X \leq_{\mathrm{NBUE}} Y \Longrightarrow X \leq_{\text {Lorenz }} Y .
$$

It is also known that (Marshall and Olkin [48], p. 69),

$$
X \leq_{\text {Lorenz }} Y \Longrightarrow c v(X) \leq c v(Y)
$$

where $c v(X)$ and $c v(Y)$ denote the coefficients of variation of $X$ and $Y$, respectively. For details on shape orders, please refer to Kochar and Wiens [38], Kochar [33], Shaked and Shanthikumar [60].

\subsubsection{Majorization orders}

We shall also be using the concept of majorization in our discussion.

Let $\left\{x_{(1)}, x_{(2)}, \cdots, x_{(n)}\right\}$ denote the increasing arrangement of the components of the vector $\mathbf{x}=\left(x_{1}, x_{2}, \cdots, x_{n}\right)$. 
Definition 1.1.13 The vector $\mathrm{x}$ is said to be majorized by the vector $\mathrm{y}$ (denoted by $\mathbf{x} \preceq_{m} \mathbf{y}$ ) if

$$
\begin{array}{r}
\qquad \sum_{i=1}^{j} x_{(i)} \geq \sum_{i=1}^{j} y_{(i)} \\
\text { for } j=1, \cdots, n-1 \text { and } \sum_{i=1}^{n} x_{(i)}=\sum_{i=1}^{n} y_{(i)} .
\end{array}
$$

Two weak majorization orders follow by relaxing the equality condition.

Definition 1.1.14 The vector $\mathrm{x}$ is said to be

- weakly supermajorized by vector $\mathbf{y}$ (denoted by $\mathbf{x} \stackrel{\text { }}{\preceq} \mathbf{y}$ ) if

$$
\sum_{i=1}^{j} x_{(i)} \geq \sum_{i=1}^{j} y_{(i)}
$$

for $j=1, \cdots, n$;

- weakly submajorized by vector $\mathbf{y}$ (denoted by $\mathbf{x} \preceq_{\mathrm{w}} \mathbf{y}$ ) if

$$
\sum_{i=1}^{j} x_{[i]} \leq \sum_{i=1}^{j} y_{[i]}
$$

for $j=1, \cdots, n$, where $\left\{x_{[1]}, x_{[2]}, \cdots, x_{[n]}\right\}$ denotes the decreasing arrangement of the components of the vector $\mathbf{x}=\left(x_{1}, x_{2}, \cdots, x_{n}\right)$.

For extensive and comprehensive details on the theory of majorization orders and their applications, please refer to the excellent book of Marshall and Olkin [47].

Another interesting weaker order related to the majorization order introduced by Bon and Păltănea [13] is the $p$ order. 
Definition 1.1.15 A vector $\mathbf{x}$ in $\mathbb{R}^{+n}$ is said to be $p$-smaller than another vector $\mathbf{y}$ in $\mathbb{R}^{+n}$ (denoted by $\mathbf{x} \stackrel{p}{\preceq} \mathbf{y}$ ) if

$$
\prod_{i=1}^{j} x_{(i)} \geq \prod_{i=1}^{j} y_{(i)}, \quad j=1, \cdots, n .
$$

Zhao and Balakrishnan [68] introduced the following reciprocal majorization order.

Definition 1.1.16 A vector $\mathrm{x}$ in $\mathbb{R}^{+n}$ is said to be reciprocally majorized by another vector $\mathbf{y}$ in $\mathbb{R}^{+n}$ (denoted by $\mathbf{x} \preceq \mathbf{r m}$ ) if

$$
\sum_{i=1}^{j} \frac{1}{x_{(i)}} \leq \sum_{i=1}^{j} \frac{1}{y_{(i)}}, \quad j=1, \cdots, n .
$$

They also wondered about the relation between $p$ order and the reciprocal order. In the following, we will answer this question.

Note that

$$
\mathbf{x} \stackrel{p}{\preceq} \mathbf{y} \Longleftrightarrow\left(\log \left(x_{1}\right), \cdots, \log \left(x_{n}\right)\right) \stackrel{\mathrm{w}}{\preceq}\left(\log \left(y_{1}\right), \cdots, \log \left(y_{n}\right)\right) .
$$

It is known that by A.2.g of Marshall and Olkin ([47], p. 117),

$$
\mathbf{x} \stackrel{\mathrm{w}}{\preceq} \mathbf{y} \Longrightarrow\left(e^{-x_{1}}, \cdots, e^{-x_{n}}\right) \preceq_{\mathrm{w}}\left(e^{-y_{1}}, \cdots, e^{-y_{n}}\right) .
$$

Combining (1.1.2) and (1.1.3), it follows that,

$$
\mathbf{x} \stackrel{p}{\preceq} \mathbf{y} \Longrightarrow\left(\frac{1}{x_{1}}, \cdots, \frac{1}{x_{n}}\right) \preceq_{\mathrm{w}}\left(\frac{1}{y_{1}}, \cdots, \frac{1}{y_{n}}\right)
$$


That is,

$$
\mathbf{x} \stackrel{p}{\preceq} \mathbf{y} \Longrightarrow \mathbf{x} \stackrel{\mathrm{rm}}{\preceq} \mathbf{y} .
$$

However, the converse is not true as pointed out in Zhao and Balakrishnan [68] through a counterexample.

Intuitively, one may consider the majorization order, $p$ order and reciprocal majorization order as the corresponding extensions of arithmetic mean, geometric mean and harmonic mean.

\subsubsection{Dependence orders}

One natural way to capture positive dependence between two random variables $X$ and $Y$ is to see if

$$
P(X \leq x, Y \leq y) \geq P(X \leq x) P(Y \leq y) \text { for all } x, y \text {. }
$$

If $(X, Y)$ satisfies the above relation, $X$ and $Y$ are said to be positively quadrant dependent (PQD) (cf. Lehmann [45]). A stronger dependence notion called right tail increasing (RTI) is defined in Barlow and Proschan [8]

$$
P(Y \leq y \mid X>x) \geq P\left(Y \leq y \mid X>x^{*}\right), \quad x \leq x^{*} .
$$

When $X$ and $Y$ satisfy the above relation, $Y$ is said to be RTI in $X$.

A further extension of RTI is the so-called stochastically increasing (SI). Random variable $Y$ is said to be $\mathrm{SI}$ in random variable $X$ if for all $y$,

$$
P(Y \leq y \mid X=x) \geq P\left(Y \leq y \mid X=x^{*}\right), \quad x \leq x^{*} .
$$


Lehmann [45] used the term, positive regression dependence to describe the SI property. For more details on dependence notions, please refer to Barlow and Proschan [8], Joe [44] and Nelsen [52].

For two pairs of random vectors $\left(X_{1}, Y_{1}\right)$ and $\left(X_{2}, Y_{2}\right)$, it will be of interest to investigate the dependence strength between them. Observing that (1.1.5) can be written as

$$
P\left(X \leq F^{-1}(u), Y \leq G^{-1}(v)\right) \geq u v, \quad u, v \in[0,1] .
$$

It is noted that

$$
C(u, v)=P\left(X \leq F^{-1}(u), Y \leq G^{-1}(v)\right)
$$

is called the copula associated with $(X, Y)$ (cf. Nelsen [52]).

Now, one natural way to compare the degree of dependence between two pairs of random variables $\left(X_{1}, Y_{1}\right)$ and $\left(X_{2}, Y_{2}\right)$ can be defined as follows.

Definition 1.1.17 $\left(X_{1}, Y_{1}\right)$ is said to be less PQD than $\left(X_{2}, Y_{2}\right)$, if and only if, for $0 \leq u \leq 1$

$$
C_{1}(u, v) \leq C_{2}(u, v)
$$

where $C_{i}(u, v)$ are associated copulas with $\left(X_{i}, Y_{i}\right)$ for $i=1,2$.

Observing that (1.1.7) can be represented as

$$
H_{\left[\xi_{q}\right]} \circ H_{\left[\xi_{p}\right]}^{-1}(u) \leq u, \quad u \in[0,1],
$$


where $\xi_{p}=F^{-1}(p)$ stands for the $p$ th quantile of the marginal distribution of $X$, and $H_{[s]}$ denotes the conditional distribution of $Y$ given $X=s$. Avérous, et al. [1] proposed the following definition to measure the relative dependence degree between two pairs of random variables.

Definition 1.1.18 $Y_{1}$ is said to be less stochastic increasing (SI) in $X_{1}$ than $Y_{2}$ is in $X_{2}$, denoted by $\left(Y_{1} \mid X_{1}\right) \prec_{\mathrm{SI}}\left(Y_{2} \mid X_{2}\right)$, if and only if, for $0 \leq u \leq 1$, and $0 \leq p \leq q \leq 1$

$$
H_{2\left[\xi_{2 q}\right]} \circ H_{2\left[\xi_{2 p}\right]}^{-1}(u) \leq H_{1\left[\xi_{1 q}\right]} \circ H_{1\left[\xi_{1 p}\right]}^{-1}(u),
$$

where $\xi_{i p}=F_{i}^{-1}(p)$ stands for the $p$ th quantile of the marginal distribution of $X_{i}$, and $H_{i[s]}$ denotes the conditional distribution of $Y_{i}$ given $X_{i}=s$, for $i=1,2$.

More SI order is a copula-based order. To see this, let $\dot{C}_{i}(u, v)=\partial C_{i}(u, v) / \partial u$ and note that by definition

$$
H_{i[s]}(t)=\dot{C}_{i}\left(F_{i}(s), G_{i}(t)\right) .
$$

For fixed $u \in(0,1)$, let $\dot{C}_{i u}^{-1}(v)$ denote the inverse of the mapping $v \mapsto$ $\dot{C}_{i}(u, v)$. Then

$$
G_{i} \circ H_{i[s]}^{-1}(u)=\dot{C}_{i F_{i}(s)}^{-1}(u)
$$

Thus if $\xi_{i p}=F_{i}^{-1}(p)$ and $\xi_{i q}=F_{i}^{-1}(q)$, one finds

$$
H_{i\left[\xi_{i q}\right]} \circ H_{i\left[\xi_{i p}\right]}^{-1}(u)=\dot{C}_{i}\left\{F_{i}\left(\xi_{i q}\right), G_{i} \circ H_{i\left[\xi_{i p}\right]}^{-1}(u)\right\}=\dot{C}_{i}\left\{q, \dot{C}_{i p}^{-1}(u)\right\},
$$


which is clearly independent of $F_{i}$ and $G_{i}$.

Recently, Dolati, et al. [18] proposed another copula-based weaker dependence order based on (1.1.6), called more RTI order.

Definition 1.1.19 $Y_{1}$ is said to be less right-tail increasing (RTI) in $X_{1}$ than $Y_{2}$ is in $X_{2}$, denoted by $\left(Y_{1} \mid X_{1}\right) \prec_{\mathrm{RTI}}\left(Y_{2} \mid X_{2}\right)$, if and only if, for $0 \leq u \leq 1$, and $0 \leq p \leq q \leq 1$

$$
H_{2\left[\xi_{2 q}\right]}^{*} \circ H_{2\left[\xi_{2 p}\right]}^{*-1}(u) \leq H_{1\left[\xi_{1 q}\right]}^{*} \circ H_{1\left[\xi_{1 p}\right]}^{*-1}(u),
$$

where $\xi_{i p}=F_{i}^{-1}(p)$ stands for the $p$ th quantile of the marginal distribution of $X_{i}$, and $H_{i[s]}^{*}$ denotes the conditional distribution of $Y_{i}$ given $X_{i}>s$, for $i=1,2$.

As mentioned in Avérous et al. [1], more PQD ordering implies

$$
\kappa\left(X_{1}, Y_{1}\right) \leq \kappa\left(X_{2}, Y_{2}\right)
$$

where $\kappa(X, Y)$ represents Spearman's rho, Kendall's tau, Gini's coefficient, or any other copula-based measure of concordance satisfying the axioms of Scarsini [57]. In the special case where $F_{1}=F_{2}$ and $G_{1}=G_{2}$, it also follows more PQD ordering implies that the pairs $\left(X_{1}, Y_{1}\right)$ and $\left(X_{2}, Y_{2}\right)$ are ordered by Pearson's correlation coefficient, namely

$$
\operatorname{corr}\left(X_{1}, Y_{1}\right) \leq \operatorname{corr}\left(X_{2}, Y_{2}\right)
$$




\section{Chapter 2}

\section{Order statistics}

\subsection{Introduction}

Order statistics have received a great amount of attention from many researchers since they are widely used in reliability, data analysis, goodness-of-fit tests, statistical inference and other applied probability and statistical areas. Most of these studies focused mainly on the case when order statistics are from independent and identically distributed (i.i.d.) random variables. Please refer to David and Nagaraja [15] and Balakrishnan and Rao [6] and [7] for more details. Studies of order statistics from heterogeneous samples began in early 70s, motivated by robustness issues. After that, much work has been done in single-outlier models or multiple-outlier models on order statistics from heterogenous samples. Balakrishnan [4] synthesized recent developments on order statistics arising from independent and non-identically distributed random variables.

In reliability engineering, an $n$ component system that works if and only if at least $k$ of the $n$ components work is called a $k$-out-of- $n$ system. Both parallel and 
series systems are special cases of the $k$-out-of- $n$ system. Let $X_{1: n} \leq X_{2: n} \leq$ $\cdots \leq X_{n: n}$ denote the order statistics of random variables $X_{1}, X_{2}, \cdots, X_{n}$. The lifetime of a $k$-out-of- $n$ system can be represented as $X_{n-k+1: n}$. It is of great interest to investigate the effect of the change in the parameters of $X_{i}$ 's on the system lifetime. Ignoring the heterogeneity leads to underestimating the average failure rate of components as pointed out in Proschan and Sethuraman [55].

Sen [58] first showed that the smallest (largest) order statistic of a sample size $n$ from heterogeneous population is stochastically smaller (larger) than the smallest (largest) order statistic of a sample of size $n$ whose distribution is the average of the distributions in the heterogeneous case. Additional results were obtained in Pledger and Proschan [54], where they assumed that the distribution functions in the heterogeneous case have proportional hazard rates (PHR).

Independent random variables $X_{1}, X_{2}, \ldots, X_{n}$ are said to follow the PHR model if for $i=1,2, \ldots, n$, the survival function of $X_{i}$ can be expressed as,

$$
\bar{F}_{i}(x)=[\bar{F}(x)]^{\lambda_{i}}, \text { for } \lambda_{i}>0,
$$

where $\bar{F}(x)$ is the survival function of some random variable $X$. If $r(t)$ denotes the hazard rate corresponding to the baseline distribution $F$, then the hazard rate of $X_{i}$ is $\lambda_{i} r(t), i=1,2, \ldots, n$. We can equivalently express (2.1.1) as

$$
\bar{F}_{i}(x)=e^{-\lambda_{i} R(x)}, i=1,2, \ldots, n
$$


where $R(x)=\int_{-\infty}^{x} r(t) d t$, is the cumulative hazard rate of $X$. Many well-known models are special cases of the PHR model. Here are some examples.

(a) Weibull: Let $R(x)=x^{\alpha}$ and $\lambda_{i}=b_{i}^{-\alpha}, \alpha>0$, then $\bar{F}_{i}(x)=\exp \left\{-\left(\frac{x}{b_{i}}\right)^{\alpha}\right\}$ is Weibull survival function with shape parameter $\alpha$ and scale parameter $b_{i}$. It is one of most widely used lifetime distributions in reliability engineering.

- Exponential: Put $R(x)=x$, then $\bar{F}_{i}(x)=e^{-\lambda_{i} x}$. It is the survival function of exponential random variable, well-known for its non-aging property in reliability theory.

- Rayleigh: Let $R(x)=x^{2}$ and $\lambda_{i}=\left(2 \sigma_{i}^{2}\right)^{-1}, \alpha>0$, then $\bar{F}_{i}(x)=$ $\exp \left\{-\frac{x^{2}}{2 \sigma_{i}^{2}}\right\}$ is Rayleigh survival function with parameter $\sigma_{i}$. It is often used to model scattered signals that reach a receiver by multiple paths in communications theory.

(b) Pareto: If $R(x)=\log (x / b)$ and $x \geq b>0$, then $\bar{F}_{i}(x)=\left(\frac{b}{x}\right)^{\lambda_{i}}$ is Pareto survival function with shape parameter $\lambda_{i}$ and scale parameter $b$, playing important roles in the field of economics since it can be used to describe the allocation of wealth among individuals.

(c) Lomax: If $R(x)=\log (1+x / b)$ and $b>0$, then $\bar{F}_{i}(x)=\left(1+\frac{x}{b}\right)^{-\lambda_{i}}$ is Lomax survival function used for stochastic modelling of decreasing failure rate life components. It is also a useful model in the study of labour turnover, biological analysis, and queueing theory. 
Theorem 2.1.1 (Pledger and Proschan [54]) If random vectors $\left(X_{1}, \cdots, X_{n}\right)$ and $\left(X_{1}^{*}, \cdots, X_{n}^{*}\right)$ have proportional hazard rate vectors $\left(\lambda_{1}, \cdots, \lambda_{n}\right)$ and $\left(\lambda_{1}^{*}, \cdots, \lambda_{n}^{*}\right)$, respectively, then, for $i=1, \cdots, n$,

$$
\left(\lambda_{1}, \cdots, \lambda_{n}\right) \succeq_{\mathrm{m}}\left(\lambda_{1}^{*}, \cdots, \lambda_{n}^{*}\right) \Rightarrow X_{i: n} \geq_{s t} X_{i: n}^{*}
$$

Subsequently, Proschan and Sethuraman [55] strengthened this result from the componentwise stochastic ordering to the multivariate stochastic ordering. That is, under the assumptions of Theorem 2.1.1, they proved that

$$
\left(X_{1: n}, \cdots, X_{n: n}\right) \stackrel{s t}{\succeq}\left(X_{1: n}^{*}, \cdots, X_{n: n}^{*}\right) .
$$

Boland et al. [10] showed with the help of a counterexample that (2.1.3) can not be strengthened from stochastic ordering to hazard rate ordering when $n \geq 3$.

Such comparisons can also be made for more general models as shown in the next theorem.

Theorem 2.1.2 (Pledger and Proschan [54]) If $\bar{F}\left(\cdot, \lambda_{i}\right)$ and $\bar{F}\left(\cdot, \lambda_{i}^{*}\right)$ are differentiable, monotone and logconvex in $\lambda_{i}$ and $\lambda_{i}^{*}$, respectively, then, for, $i=1, \cdots, n$,

$$
\left(\lambda_{1}, \cdots, \lambda_{n}\right) \succeq_{\mathrm{m}}\left(\lambda_{1}^{*}, \cdots, \lambda_{n}^{*}\right) \Rightarrow X_{i: n} \geq_{s t} X_{i: n}^{*}
$$

A familiar example of a class of distributions satisfying the hypothesis of Theorem 2.1.2 is when

$$
\bar{F}\left(x, \lambda_{i}\right)=\bar{F}\left(\lambda_{i} x\right),
$$


with distribution $F$ having decreasing failure rate (DFR) as a DFR survival probability is logconvex (cf. Barlow and Proschan [8]).

This topic is followed up by Dykstra et al. [19] where they showed that if $X_{1}, \ldots, X_{n}$ are independent exponential random variables with $X_{i}$ having hazard rate $\lambda_{i}, i=1, \ldots, n$, and $Y_{1}, \ldots, Y_{n}$ is a random sample of size $n$ from an exponential distribution with common hazard rate $\bar{\lambda}=\sum_{i=1}^{n} \lambda_{i} / n$, then

$$
Y_{n: n} \leq_{h r} X_{n: n} \quad \text { and } \quad Y_{n: n} \leq_{\text {disp }} X_{n: n} .
$$

Under a weaker condition that if $Y_{1}, \ldots, Y_{n}$ is a random sample with common hazard rate $\tilde{\lambda}=\left(\prod_{i=1}^{n} \lambda_{i}\right)^{1 / n}$, the geometric mean of $\lambda_{i}$ 's, Khaledi and Kochar [27] proved that,

$$
Y_{n: n} \leq_{h r} X_{n: n} \quad \text { and } \quad Y_{n: n} \leq_{d i s p} X_{n: n}
$$

They also showed there that

$$
\left(\lambda_{1}, \lambda_{2}, \cdots, \lambda_{n}\right) \succeq\left(\lambda_{1}^{*}, \lambda_{2}^{*}, \cdots, \lambda_{n}^{*}\right) \Rightarrow X_{n: n} \geq_{s t} X_{n: n}^{*}
$$

which improves the bound given by (2.1.3). Later, Khaledi and Kochar [29] extended the results (2.1.7) and (2.1.8) from the exponential case to the PHR model.

Theorem 2.1.3 Let $X_{1}, \ldots, X_{n}$ be independent random variables with $X_{i}$ having survival function $\bar{F}^{\lambda_{i}}, i=1, \ldots, n$. Let $Y_{1}, \ldots, Y_{n}$ be a random sample with common population survival distribution $\bar{F}^{\tilde{\lambda}}$, where $\tilde{\lambda}=\left(\prod_{i=1}^{n} \lambda_{i}\right)^{1 / n}$, then 
(i) $Y_{n: n} \leq_{h r} X_{n: n}$;

(ii) $Y_{n: n} \leq_{\text {disp }} X_{n: n}$ if $F$ is of decreasing hazard rate (DFR).

These results give nice bounds for variances of parallel systems with components which are independent following the PHR model in terms of the case when they are i.i.d..

Theorem 2.1.4 If $X_{1}, \ldots, X_{n}$ are independent random variables with $X_{i}$ having survival function $\bar{F}^{\lambda_{i}}, i=1, \ldots, n$, and if $X_{1}^{*}, \ldots, X_{n}^{*}$ be another random sample with $X_{i}^{*}$ having survival distribution $\bar{F}^{\lambda_{i}^{*}}, i=1, \ldots, n$. Then

$$
\left(\lambda_{1}, \lambda_{2}, \cdots, \lambda_{n}\right) \stackrel{p}{\succeq}\left(\lambda_{1}^{*}, \lambda_{2}^{*}, \cdots, \lambda_{n}^{*}\right) \Rightarrow X_{n: n} \geq_{s t} X_{n: n}^{*}
$$

Khaledi and Kochar [29] also showed that Theorem 2.1.4 may not hold for other order statistics.

Bon and Păltănea [14] showed that if $X_{1}, \ldots, X_{n}$ are independent exponential random variables with $X_{i}$ having hazard rate $\lambda_{i}$ for $i=1, \ldots, n$, and $Y_{1}, \ldots, Y_{n}$ is a random sample of size $n$ from an exponential distribution with common hazard rate $\lambda$, then

$$
Y_{k: n} \leq_{s t} X_{k: n} \Longleftrightarrow \lambda \geq\left(\left(\begin{array}{l}
n \\
k
\end{array}\right)^{-1} \sum_{1 \leq i_{1}<\ldots<i_{k} \leq n} \lambda_{i_{1}} \ldots \lambda_{i_{k}}\right)^{1 / k} .
$$




\subsection{Stochastic comparisons of parallel systems by likelihood ratio order}

It is an open problem in the literature whether the relationship in (2.1.6) could be strengthened to the likelihood ratio order in the PHR model? I will answer the question in this section.

The following two lemmas will be used to prove our main result.

Lemma 2.2.1 (Khaledi and Kochar [27]) For $x \geq 0$, the functions

$$
\frac{1-e^{-x}}{x} \text { and } \frac{x^{2} e^{-x}}{\left(1-e^{-x}\right)^{2}}
$$

are both decreasing.

Lemma 2.2.2 Let $X_{1}, \ldots, X_{n}$ be independent exponential random variables with $X_{i}$ having hazard rate $\lambda_{i}, i=1, \ldots, n$. Let $Y_{1}, \ldots, Y_{n}$ be a random sample of size $n$ from an exponential distribution with common hazard rate $\bar{\lambda}=\sum_{i=1}^{n} \lambda_{i} / n$. Then,

$$
Y_{n: n} \leq_{l r} X_{n: n}
$$

Proof: For $x \geq 0$, the distribution function of $X_{n: n}$ is,

$$
F_{n: n}(x)=\prod_{i=1}^{n}\left(1-e^{-\lambda_{i} x}\right)
$$

with density function as

$$
f_{n: n}(x)=F_{n: n}(x) \sum_{i=1}^{n} \frac{\lambda_{i} e^{-\lambda_{i} x}}{1-e^{-\lambda_{i} x}} .
$$


Similarly, the distribution function of $Y_{n: n}$ for $x \geq 0$ is,

$$
G_{n: n}(x)=\left(1-e^{-\bar{\lambda} x}\right)^{n},
$$

with density function

$$
g_{n: n}(x)=G_{n: n}(x) \frac{n \bar{\lambda} e^{-\bar{\lambda} x}}{1-e^{-\bar{\lambda} x}}
$$

Note that, for $x \geq 0$,

$$
\frac{f_{n: n}(x)}{g_{n: n}(x)}=\frac{\sum_{i=1}^{n} \frac{\lambda_{i} e^{-\lambda_{i} x}}{1-e^{-\lambda_{i} x}}}{\frac{n \bar{\lambda} e^{-\bar{\lambda} x}}{1-e^{-\bar{\lambda} x}}} \frac{F_{n: n}(x)}{G_{n: n}(x)}=\frac{h_{1}(x)}{n \bar{\lambda}} \frac{F_{n: n}(x)}{G_{n: n}(x)},
$$

where

$$
h_{1}(x)=\frac{\sum_{i=1}^{n} \frac{\lambda_{i} e^{-\lambda_{i} x}}{1-e^{-\lambda_{i} x}}}{\frac{e^{-\bar{\lambda} x}}{1-e^{-\bar{\lambda} x}}}=\sum_{i=1}^{n} \lambda_{i} \frac{e^{\bar{\lambda} x}-1}{e^{\lambda_{i} x}-1}
$$

Since

$$
(\bar{\lambda}, \ldots, \bar{\lambda}) \preceq_{\mathrm{m}}\left(\lambda_{1}, \cdots, \lambda_{n}\right),
$$

it follows from Theorem 3.2 of Dykstra et al. [19] that

$$
\frac{F_{n: n}(x)}{G_{n: n}(x)}
$$

is increasing in $x \geq 0$. Thus, it is sufficient to prove that $h_{1}(x)$ is increasing in $x \geq 0$.

The derivative of $h_{1}(x)$ is, for $x \geq 0$,

$$
h_{1}^{\prime}(x)=\bar{\lambda} e^{\bar{\lambda} x} \sum_{i=1}^{n} \frac{\lambda_{i}}{e^{\lambda_{i} x}-1}-\left(e^{\bar{\lambda} x}-1\right) \sum_{i=1}^{n} \frac{\lambda_{i}^{2} e^{\lambda_{i} x}}{\left(e^{\lambda_{i} x}-1\right)^{2}} .
$$


By Lemma 2.2.1 and Čebyšev's sum inequality (Mitrinović [49], Theorem 1, p.36), it holds that, for $x \geq 0$,

$$
\bar{\lambda} e^{\bar{\lambda} x} \sum_{i=1}^{n} \frac{\lambda_{i}}{e^{\lambda_{i} x}-1} \geq \frac{\bar{\lambda} e^{\bar{\lambda} x}}{n} \sum_{i=1}^{n} \frac{\lambda_{i}^{2} e^{-\lambda_{i} x}}{\left(1-e^{-\lambda_{i} x}\right)^{2}} \sum_{i=1}^{n} \frac{1-e^{-\lambda_{i} x}}{\lambda_{i}} .
$$

Thus, $h_{1}^{\prime}(x)$ will be nonnegative if, for $x \geq 0$,

$$
\frac{\bar{\lambda} e^{\bar{\lambda} x}}{n} \sum_{i=1}^{n} \frac{1-e^{-\lambda_{i} x}}{\lambda_{i}} \geq e^{\bar{\lambda} x}-1
$$

holds.

Denote, for $x \geq 0$,

$$
h_{2}(x)=\frac{\bar{\lambda}}{n} \sum_{i=1}^{n} \frac{1-e^{-\lambda_{i} x}}{\lambda_{i}}-\left(1-e^{-\bar{\lambda} x}\right) .
$$

Since the derivative of $h_{2}(x)$ is,

$$
h_{2}^{\prime}(x)=\frac{\bar{\lambda}}{n} \sum_{i=1}^{n} e^{-\lambda_{i} x}-\bar{\lambda} e^{-\bar{\lambda} x}
$$

and by the arithmetic-geometric mean inequality, for $x \geq 0$,

$$
\frac{\sum_{i=1}^{n} e^{-\lambda_{i} x}}{n} \geq \sqrt[n]{\prod_{i=1}^{n} e^{-\lambda_{i} x}}=e^{-\bar{\lambda} x}
$$

it follows that $h_{2}^{\prime}(x) \geq 0$ for $x \geq 0$. That is, $h_{2}(x)$ is increasing in $x \geq 0$. Observing that $h_{2}(0)=0$, we have $h_{2}(x) \geq 0$ for $x \geq 0$. Hence, $h_{1}(x)$ is increasing in $x \geq 0$. The required result follows immediately.

Now, we are ready to extend the above result to the PHR family. 
Theorem 2.2.3 Let $X_{1}, \ldots, X_{n}$ be independent random variables with $X_{i}$ having survival function $\bar{F}^{\lambda_{i}}, i=1, \ldots, n$. Let $Y_{1}, \ldots, Y_{n}$ be a random sample with common population survival distribution $\bar{F}^{\bar{\lambda}}$, where $\bar{\lambda}=\sum_{i=1}^{n} \lambda_{i} / n$, then

$$
Y_{n: n} \leq_{l r} X_{n: n}
$$

Proof: Note that the cumulative hazard of $F$ is,

$$
H(x)=-\log \bar{F}(x)
$$

Now for $x \geq 0, i=1, \ldots, n$,

$$
P\left(H\left(X_{i}\right)>x\right)=P\left(X_{i}>H^{-1}(x)\right)=\bar{F}^{\lambda_{i}}\left(\bar{F}^{-1}\left(e^{-x}\right)\right)=e^{-\lambda_{i} x},
$$

where $H^{-1}$ is the right inverse of $H$. Denoting by $X_{i}^{\prime}=H\left(X_{i}\right)$, we notice that $X_{i}^{\prime}$ is exponential with hazard rate $\lambda_{i}$ for $i=1, \ldots, n$. Similarly, let $Y_{i}^{\prime}=H\left(Y_{i}\right)$, is exponential with hazard rate $\bar{\lambda}$ for $i=1, \ldots, n$. It follows from Lemma 2.2.2,

$$
Y_{n: n}^{\prime} \leq_{l r} X_{n: n}^{\prime}
$$

That is,

$$
H\left(Y_{n: n}\right) \leq_{l r} H\left(X_{n: n}\right) .
$$

Since $H^{-1}$ is an increasing function, it follows from Theorem 1.C.4 in Shaked and Shanthikumar [60] that,

$$
Y_{n: n} \leq_{l r} X_{n: n}
$$


One may wonder whether (2.1.7) of Khaledi and Kochar [27] can be strengthened from the hazard rate order to the likelihood ratio order. The following example serves as a counterexample.

Example 2.2.4 Let $X_{1}, \ldots, X_{n}$ be independent exponential random variables with $X_{i}$ having hazard rate $\lambda_{i}, i=1, \ldots, n$, and $Z_{1}, \ldots, Z_{n}$ be a random sample of size $n$ from an exponential distribution with common hazard rate $\tilde{\lambda}=\left(\prod_{i=1}^{n} \lambda_{i}\right)^{1 / n}$. Then, the reversed hazard rate of $X_{n: n}$ is

$$
\frac{f_{n: n}(x)}{F_{n: n}(x)}=\sum_{i=1}^{n} \frac{\lambda_{i} e^{-\lambda_{i} x}}{1-e^{-\lambda_{i} x}}
$$

Similarly, the reversed hazard rate of $Z_{n: n}$ is

$$
\frac{g_{n: n}(x)}{G_{n: n}(x)}=n \tilde{\lambda} \frac{e^{-\tilde{\lambda} x}}{1-e^{-\tilde{\lambda} x}}
$$

Let $\lambda_{1}=\lambda_{2}=1, \lambda_{3}=3$ and $n=3$, then,

$$
\frac{f_{n: n}(1)}{F_{n: n}(1)} \approx 1.321 \leq 1.339 \approx \frac{g_{n: n}(1)}{G_{n: n}(1)}
$$

Thus,

$$
X_{n: n} \Varangle_{r h} Z_{n: n},
$$

which implies that

$$
X_{n: n} \nsupseteq l r Z_{n: n} .
$$


Remark: Remark 2.2 of Khaledi and Kochar [27] asserted that the stochastic order in (2.1.8) can not be extended to the hazard rate order. Example 2.2.4 above also shows that

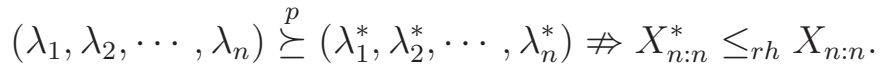

\subsection{Comparisons of parallel systems according to convex transform order}

\subsubsection{Introduction}

In statistics, skewness describes the departure of a density from symmetry, where one tail of the density is "stretched out" more than the other. Several well-known measures of skewness are available in the statistics literature, such as Pearson's coefficient of skewness and Edgeworth's coefficient. Interested readers may refer to Arnold and Groeneveld [3] and Marshall and Olkin ([48], p.70) for more discussion and other measures of skewness. In the case of unimodal distributions, if a density is to the left of "center" and the right tail is relatively long, then the density is said to be skewed to the right. This kind of skewness is used when the distributions have nonnegative support. It is of interest to compare the skewness of two distributions. van Zwet [63] proposed a skewness order, called the convex transform order, which captures the essence of what one distribution is more skewed than the other. This order is also an important concept in reliability theory, since it reflects one distribution being more IFR (increasing failure rate). 
Series and parallel systems are the building blocks of more complex coherent systems. Note that when the components are independent, the hazard rate of a series system is the sum of the hazard rates of the components. In particular, if the components are identically and identically distributed, the aging properties of a series system will be the same as those of the components. But this is not the case with parallel systems. In fact, it is well known that the lifetime of a parallel system with independent IFR components may not be IFR if they are not identically distributed.

Consider two parallel systems with independent exponential components, the first with identical components and the second with nonidentical components. Many authors have studied the magnitude (stochastic) and variability orderings of such systems when the parameters of the exponential distributions satisfy certain restrictions (cf. Section 2.1).

In this section, I will compare such systems from a different perspective of statistics through the skewness order. More precisely, two such systems will be compared according to convex transform order which compares the shapes of their probability distributions. As we discuss below, it can also be interpreted as comparing the relative aging of two distributions. This problem has not been studied in the literature and the purpose of this section is to fix this gap. Intuitively, the density function of the largest order statistic from a heterogenous sample will be more skewed than one from a homogeneous sample. This will be confirmed ac- 
cording to convex transform order for exponential samples. While the previous work mentioned above gives us bounds on measures of variability, like variance of the largest order statistic in terms of the i.i.d. case, the new result will give us bounds on measures of skewness, like coefficient of variation. It will be shown that the coefficient of variation of the largest order statistic of $n$ independent and non identically distributed exponential random variables satisfies the inequality,

$$
c v\left(X_{n: n}\right) \geq\left(\sqrt{\sum_{i=1}^{n} \frac{1}{i^{2}}}\right) /\left(\sum_{i=1}^{n} \frac{1}{i}\right) .
$$

Using the main result, in Proposition 2.3.3, an equivalent characterization of dispersion relation in (2.1.6) is given. It is proved that the dispersive order there is equivalent to the usual stochastic order, which gives us a deeper understanding of the result in Khaledi and Kochar [27]. An equivalent characterization for comparing systems in terms of right spread order, which can be considered as the second variability order, is also derived as a consequence.

\subsubsection{Which parallel system ages faster?}

Suppose we have two parallel systems, one with independent and identically distributed exponential components and the other with heterogenous exponential components. The following result shows that a parallel system with homogenous exponential components ages faster than the one with heterogeneous exponential components in the sense of convex transform order. 
Theorem 2.3.1 Let $X_{1}, \ldots, X_{n}$ be independent exponential random variables with $X_{i}$ having hazard rate $\lambda_{i}, i=1, \ldots, n$. Let $Y_{1}, \ldots, Y_{n}$ be a random sample of size $n$ from an exponential distribution with common hazard rate $\lambda$. Then,

$$
Y_{n: n} \leq_{c} X_{n: n}
$$

Proof: Let $G$ and $F$ denote the distribution functions of $Y_{n: n}$ and $X_{n: n}$ with corresponding density functions $g$ and $f$, respectively.

Then for $x \geq 0$,

$$
G(x)=P\left(Y_{n: n} \leq x\right)=\left(1-e^{-\lambda x}\right)^{n},
$$

and

$$
F(x)=P\left(X_{n: n} \leq x\right)=\prod_{i=1}^{n}\left(1-e^{-\lambda_{i} x}\right) .
$$

From Proposition 21.A.7 of Marshall and Olkin [48], it is sufficient to show $G^{-1} F(x)$ is concave on $(0, \infty)$. Note that, for $x \geq 0$,

$$
G^{-1} F(x)=-\frac{1}{\lambda} \ln \left(1-F^{1 / n}(x)\right)=-\frac{1}{\lambda} \ln \left[1-\prod_{i=1}^{n}\left(1-e^{-\lambda_{i} x}\right)^{1 / n}\right] .
$$

Hence,

$$
g\left[G^{-1} F(x)\right]=n \lambda\left[1-\prod_{i=1}^{n}\left(1-e^{-\lambda_{i} x}\right)^{1 / n}\right] \prod_{i=1}^{n}\left(1-e^{-\lambda_{i} x}\right)^{(n-1) / n}
$$


Differentiating with respect to $x$, we get

$$
\begin{aligned}
{\left[G^{-1} F(x)\right]^{\prime} } & =\frac{f(x)}{g\left[G^{-1} F(x)\right]} \\
& =\frac{\sum_{i=1}^{n} \frac{\lambda_{i} e^{-\lambda_{i} x}}{1-e^{-\lambda_{i} x}} \prod_{i=1}^{n}\left(1-e^{-\lambda_{i} x}\right)}{n \lambda\left[1-\prod_{i=1}^{n}\left(1-e^{-\lambda_{i} x}\right)^{1 / n}\right] \prod_{i=1}^{n}\left(1-e^{-\lambda_{i} x}\right)^{(n-1) / n}} \\
& =\frac{\sum_{i=1}^{n} \frac{\lambda_{i} e^{-\lambda_{i} x}}{1-e^{-\lambda_{i} x}} \prod_{i=1}^{n}\left(1-e^{-\lambda_{i} x}\right)^{1 / n}}{n \lambda\left[1-\prod_{i=1}^{n}\left(1-e^{-\lambda_{i} x}\right)^{1 / n}\right]} .
\end{aligned}
$$

So, it is enough to prove that

$$
\begin{aligned}
h(x) & =\frac{\sum_{i=1}^{n} \frac{\lambda_{i} e^{-\lambda_{i} x}}{1-e^{-\lambda_{i} x}}}{\prod_{i=1}^{n}\left(1-e^{-\lambda_{i} x}\right)^{-1 / n}-1} \\
& =\frac{\sum_{i=1}^{n} \frac{\lambda_{i}}{e^{\lambda_{i} x}-1}}{\prod_{i=1}^{n}\left(1-e^{-\lambda_{i} x}\right)^{-1 / n}-1}
\end{aligned}
$$

is decreasing in $x \geq 0$. By taking the derivative, it is equivalent to showing that

$$
\begin{aligned}
& \sum_{i=1}^{n} \frac{\lambda_{i}^{2} e^{\lambda_{i} x}}{\left(e^{\lambda_{i} x}-1\right)^{2}}\left[\prod_{i=1}^{n}\left(1-e^{-\lambda_{i} x}\right)^{-1 / n}-1\right] \\
\geq & \frac{1}{n} \sum_{i=1}^{n} \frac{\lambda_{i}}{e^{\lambda_{i} x}-1} \sum_{i=1}^{n} \frac{\lambda_{i} e^{-\lambda_{i} x}}{1-e^{-\lambda_{i} x}} \prod_{i=1}^{n}\left(1-e^{-\lambda_{i} x}\right)^{-1 / n},
\end{aligned}
$$

i.e.,

$$
\sum_{i=1}^{n} \frac{\lambda_{i}^{2} e^{-\lambda_{i} x}}{\left(1-e^{-\lambda_{i} x}\right)^{2}}\left[1-\prod_{i=1}^{n}\left(1-e^{-\lambda_{i} x}\right)^{1 / n}\right] \geq \frac{1}{n}\left(\sum_{i=1}^{n} \frac{\lambda_{i} e^{-\lambda_{i} x}}{1-e^{-\lambda_{i} x}}\right)^{2} .
$$


From the classical Cauchy-Schwarz inequality (Mitrinović [49], Theorem 1, p. 41), it follows that, for $x \geq 0$,

$$
\sum_{i=1}^{n} \frac{\lambda_{i}^{2} e^{-\lambda_{i} x}}{\left(1-e^{-\lambda_{i} x}\right)^{2}} \sum_{i=1}^{n} e^{-\lambda_{i} x} \geq\left(\sum_{i=1}^{n} \frac{\lambda_{i} e^{-\lambda_{i} x}}{1-e^{-\lambda_{i} x}}\right)^{2} .
$$

Hence, it is sufficient to show that, for $x \geq 0$,

$$
\sum_{i=1}^{n} \frac{\lambda_{i}^{2} e^{-\lambda_{i} x}}{\left(1-e^{-\lambda_{i} x}\right)^{2}}\left[1-\prod_{i=1}^{n}\left(1-e^{-\lambda_{i} x}\right)^{1 / n}\right] \geq \frac{1}{n} \sum_{i=1}^{n} \frac{\lambda_{i}^{2} e^{-\lambda_{i} x}}{\left(1-e^{-\lambda_{i} x}\right)^{2}} \sum_{i=1}^{n} e^{-\lambda_{i} x}
$$

i.e.,

$$
\frac{1}{n} \sum_{i=1}^{n}\left(1-e^{-\lambda_{i} x}\right) \geq \prod_{i=1}^{n}\left(1-e^{-\lambda_{i} x}\right)^{1 / n},
$$

which is guarantied by the arithmetic-geometric mean inequality.

Remark 1: Theorem 2.3.1 reveals that a parallel system with homogeneous exponential components ages faster than than a system with heterogenous exponential components in the sense of "more IFR" property. Note that a parallel system with homogeneous exponential components is IFR (Barlow and Proschan [8]). However, a parallel system with heterogenous exponential components may not be IFR.

Remark 2: It is interesting to note that, unlike the magnitude and variability orders, no restriction on the parameters is needed for Theorem 2.3.1 to hold as the convex transform order is scale invariant. Intuitively, due to the heterogeneity, the largest order statistic from a heterogenous sample will be more skewed than that 
from a homogeneous sample. Theorem 2.3.1 confirms this fact for exponential samples.

In Figure 1, we plot the densities of three parallel systems with independent exponential components and with parameters $(2,6,9),(4,5,8)$ and $(17 / 3,17 / 3,17 / 3)$, respectively. It is easy to verify that

$$
(17 / 3,17 / 3,17 / 3) \preceq_{\mathrm{m}}(4,5,8) \preceq_{\mathrm{m}}(2,6,9)
$$

in the majorization ordering of Marshall and Olkin [47]. Let $X_{1}, \ldots, X_{n}$ be independent exponential random variables with $X_{i}$ having hazard rate $\lambda_{i}, i=1, \ldots, n$, and let $Y_{1}, \ldots, Y_{n}$ be a random samples of size $n$ from an exponential distribution with $Y_{i}$ having hazard rate $\theta_{i}, i=1, \ldots, n$, such that

$$
\left(\theta_{1}, \cdots, \theta_{n}\right) \preceq_{\mathrm{m}}\left(\lambda_{1}, \cdots, \lambda_{n}\right)
$$

Looking at Figure 1, one may wonder whether in this case

$$
Y_{n: n} \leq_{c} X_{n: n}
$$

Though we believe this result to be true from empirical evidence, we are unable to establish it mathematically so far.

The following result, which is of independent interest in economics, is a direct consequence of Theorem 2.3.1

Corollary 2.3.2 Let $X_{1}, \ldots, X_{n}$ be independent exponential random variables 


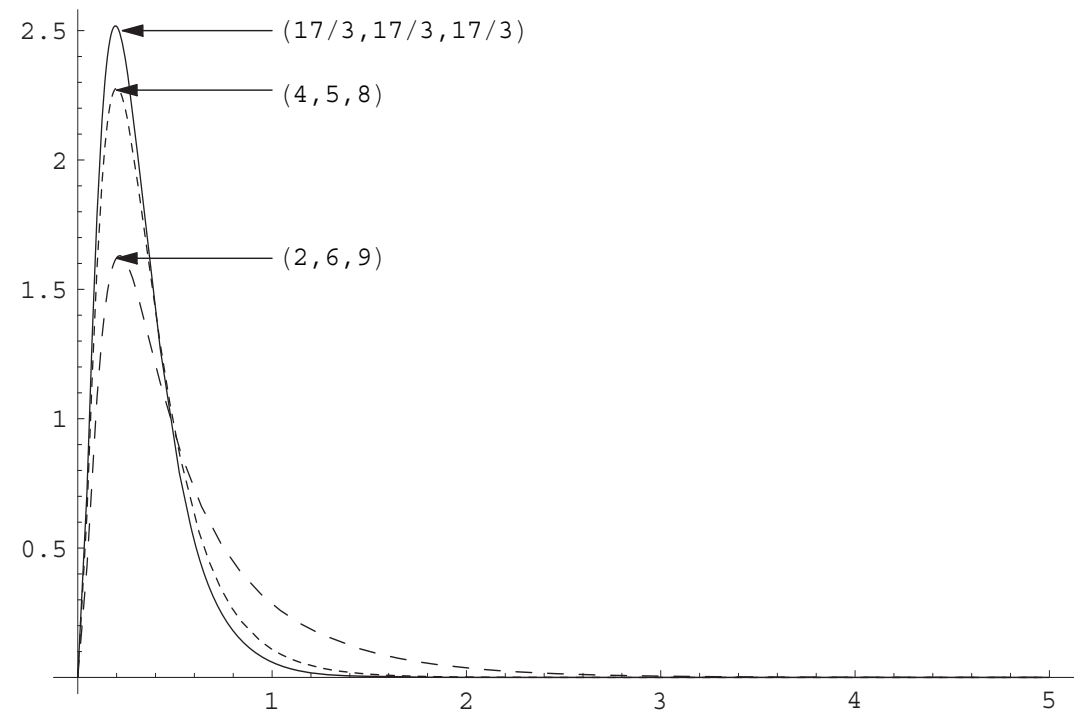

Figure 2.1: The density curves with different parameters.

with $X_{i}$ having hazard rate $\lambda_{i}, i=1, \ldots, n$. Let $Y_{1}, \ldots, Y_{n}$ be a random sample of size $n$ from an exponential distribution with common hazard rate $\lambda$. Then,

$$
Y_{n: n} \leq_{\text {Lorenz }} X_{n: n}
$$

From Barlow and Proschan ([8], p. 60), it follows that,

$$
\mathrm{E} Y_{n: n}=\frac{1}{\lambda} \sum_{i=1}^{n} \frac{1}{i}
$$

and

$$
\operatorname{Var}\left(Y_{n: n}\right)=\frac{1}{\lambda^{2}} \sum_{i=1}^{n} \frac{1}{i^{2}} .
$$

Using (1.1.1), one get the following lower bound on the coefficient of variation of $X_{n: n}$, 


$$
c v\left(X_{n: n}\right) \geq\left(\sqrt{\sum_{i=1}^{n} \frac{1}{i^{2}}}\right) /\left(\sum_{i=1}^{n} \frac{1}{i}\right) .
$$

\subsubsection{Dispersion}

The next result gives equivalent characterizations of the dispersive order and the right spread order between two parallel systems.

Proposition 2.3.3 Let $X_{1}, \ldots, X_{n}$ be independent exponential random variables with $X_{i}$ having hazard rate $\lambda_{i}, i=1, \ldots, n$ and let $Y_{1}, \ldots, Y_{n}$ be a random sample of size $n$ from an exponential distribution with common hazard rate $\lambda$. Then,

i) $Y_{n: n} \leq_{d i s p} X_{n: n} \Longleftrightarrow Y_{n: n} \leq_{s t} X_{n: n}$;

ii) $Y_{n: n} \leq_{R S} X_{n: n} \Longleftrightarrow \mathrm{E} Y_{n: n} \leq \mathrm{E} X_{n: n}$.

Proof: i) Ahmed et al. [2] proved in their Theorem 3 that the conditions $Y \leq_{s u} X$ and $Y \leq_{s t} X$ imply $Y \leq_{d i s p} X$. Since $Y_{n: n} \leq_{c} X_{n: n}$ implies $Y_{n: n} \leq_{s u} X_{n: n}$, it follows that the condition $Y_{n: n} \leq_{s t} X_{n: n}$ will imply $Y_{n: n} \leq_{d i s p} X_{n: n}$. Conversely, observing that $Y_{n: n} \leq_{d i s p} X_{n: n}$ implies $Y_{n: n} \leq_{s t} X_{n: n}$ in case of distributions with a common left hand point of the support, the required result follows immediately from Theorem 2.3.1.

ii) Theorem 4.3 in Fernández-Ponce, et al. [20] shows $Y \leq_{\mathrm{NBUE}} X$ and $\mathrm{E} Y \leq$ E $X$ imply $Y \leq_{R S} X$. Note that $Y_{n: n} \leq_{c} X_{n: n}$ implies $Y_{n: n} \leq_{\text {NBUE }} X_{n: n}$ and since 
$Y_{n: n} \leq_{R S} X_{n: n}$ implies $\mathrm{E} Y_{n: n} \leq \mathrm{E} X_{n: n}$, the result follows from Theorem 2.3.1 again.

Khaledi and Kochar [27] proved that

$$
\lambda=\left(\prod_{i=1}^{n} \lambda_{i}\right)^{1 / n} \Longrightarrow Y_{n: n} \leq_{d i s p} X_{n: n} .
$$

Actually, one may easily check that

$$
\lambda=\left(\prod_{i=1}^{n} \lambda_{i}\right)^{1 / n} \leq \lambda \Longleftrightarrow Y_{n: n} \leq_{\text {disp }} X_{n: n} .
$$

Now, one question arises naturally: what is the equivalent condition for the right spread order between these two parallel systems? This question has been lying unanswered for a long time since it is very complicated to check it from the definition of the right spread order. However, using Proposition 2.3.3, one could easily solve this problem.

Proposition 2.3.4 Let $X_{1}, \ldots, X_{n}$ be independent exponential random variables with $X_{i}$ having hazard rate $\lambda_{i}, i=1, \ldots, n$. Let $Y_{1}, \ldots, Y_{n}$ be a random sample of size $n$ from an exponential distribution with common hazard rate $\lambda$. Then,

$$
\lambda^{*} \leq \lambda \Longleftrightarrow Y_{n: n} \leq_{R S} X_{n: n}
$$

where

$$
\lambda^{*}=\sum_{i=1}^{n} \frac{1}{i}\left[\sum_{k=1}^{n}(-1)^{k+1} \sum_{1 \leq i_{1} \leq \cdots \leq i_{k} \leq n} \frac{1}{\sum_{j=1}^{k} \lambda_{i_{j}}}\right]^{-1} .
$$


Proof: Note that

$$
\mathrm{E} Y_{n: n}=\frac{1}{\lambda} \sum_{i=1}^{n} \frac{1}{i}
$$

and, for $x \geq 0$,

$$
\begin{aligned}
\mathrm{E} X_{n: n} & =\int_{0}^{\infty}\left[1-\prod_{i=1}^{n}\left(1-e^{-\lambda_{i} x}\right)\right] d x \\
& =\int_{0}^{\infty}\left[\sum_{k=1}^{n}(-1)^{k+1} \sum_{1 \leq i_{1} \leq \cdots \leq i_{k} \leq n} e^{-\sum_{j=1}^{k} \lambda_{i_{j}} x}\right] d x \\
& =\sum_{k=1}^{n}(-1)^{k+1} \sum_{1 \leq i_{1} \leq \cdots \leq i_{k} \leq n} \frac{1}{\sum_{j=1}^{k} \lambda_{i_{j}}}
\end{aligned}
$$

Hence, the result follows immediately from Proposition 2.3.3 (ii).

From (2.3.5) and (2.3.6), it is seen that $\lambda^{*} \leq \tilde{\lambda}$ as the dispersive order implies the right spread order. Although it is complicated to compute $\lambda^{*}$, Proposition 2.3.4 is very useful since $\mathrm{E} X_{n: n}$ could be either easily numerically computed or derived directly from the data. The following example gives a good illustration.

Example 2.3.5 Let $\lambda_{1}=2, \lambda_{2}=4$ and $\lambda_{3}=5$. Now,

$$
\mathrm{E} X_{3: 3}=\int_{0}^{\infty}\left[1-\prod_{i=1}^{n}\left(1-e^{-\lambda_{i} x}\right)\right] d x=\frac{8597}{13860}
$$

Hence,

$$
\lambda^{*}=\frac{13860}{8597}\left(1+\frac{1}{2}+\frac{1}{3}\right)=2.95568 .
$$


However,

$$
\tilde{\lambda}=(40)^{1 / 3}=3.41995
$$

So $\lambda^{*}<\tilde{\lambda}$.

Relation (2.3.4) has been extended to the proportional hazard rate models by Khaledi and Kochar [29]. Let $X_{1}, \ldots, X_{n}$ be independent random variables with $X_{i}$ having survival function $\bar{F}^{\lambda_{i}}, i=1, \ldots, n$, and let $Y_{1}, \ldots, Y_{n}$ be a random sample from a distribution with the common survival distribution $\bar{F}^{\lambda}$. Khaledi and Kochar [29] have shown that if $F$ is DFR (decreasing failure rate), then

$$
\lambda=\left(\prod_{i=1}^{n} \lambda_{i}\right)^{1 / n} \Longrightarrow Y_{n: n} \leq_{d i s p} X_{n: n}
$$

which actually could be written as

$$
\lambda \geq\left(\prod_{i=1}^{n} \lambda_{i}\right)^{1 / n} \Longrightarrow Y_{n: n} \leq_{d i s p} X_{n: n} .
$$

Motivated by this observation, Proposition 2.3.4 is extended to the proportional hazard rate models.

The following lemma due to Kochar et al. [37] will be used to prove the result.

Lemma 2.3.6 Let $X$ and $Y$ be continuous random variables with finite means, then for any increasing and convex function $\phi$, it holds that

$$
Y \leq_{R S} X \Longrightarrow \phi(Y) \leq_{R S} \phi(X)
$$


Theorem 2.3.7 Let $X_{1}, \ldots, X_{n}$ be independent random variables with $X_{i}$ having survival function $\bar{F}^{\lambda_{i}}, i=1, \ldots, n$, and let $Y_{1}, \ldots, Y_{n}$ be another random sample with the common survival function $\bar{F}^{\lambda}$. If $F$ is DFR, then

$$
\lambda^{*} \leq \lambda \Longrightarrow Y_{n: n} \leq_{R S} X_{n: n}
$$

where $\lambda^{*}$ is given in (2.3.6).

Proof: The proof is similar to Theorem 2.2.3. Note that the cumulative hazard of $F$ is,

$$
H(x)=-\log \bar{F}(x)
$$

Now for $x \geq 0, i=1, \ldots, n$,

$$
P\left(H\left(X_{i}\right)>x\right)=P\left(X_{i}>H^{-1}(x)\right)=\bar{F}^{\lambda_{i}}\left(\bar{F}^{-1}\left(e^{-x}\right)\right)=e^{-\lambda_{i} x},
$$

where $H^{-1}$ is the right inverse of $H$. Denoting by $X_{i}^{\prime}=H\left(X_{i}\right)$, we notice that $X_{i}^{\prime}$ is exponential with hazard rate $\lambda_{i}$ for $i=1, \ldots, n$. Similarly, let $Y_{i}^{\prime}=H\left(Y_{i}\right)$, be exponential with hazard rate $\lambda$ for $i=1, \ldots, n$. It follows from (2.3.6), for $\lambda^{*} \leq \lambda$,

$$
Y_{n: n}^{\prime} \leq_{R S} X_{n: n}^{\prime}
$$

That is,

$$
H\left(Y_{n: n}\right) \leq_{R S} H\left(X_{n: n}\right)
$$

Since $F$ is DFR, $H(\cdot)$ is increasing and concave, which implies $H^{-1}(\cdot)$ is increasing and convex. The required result follows from Lemma 2.3.6. 
As a consequence, we have the following result.

Corollary 2.3.8 Let $X_{1}, \ldots, X_{n}$ be independent random variables with $X_{i}$ having survival function $\bar{F}^{\lambda_{i}}, i=1, \ldots, n$, and let $Y_{1}, \ldots, Y_{n}$ be another random sample with the common survival function $\bar{F}^{\lambda}$. If $F$ is DFR, then

$$
\lambda^{*} \leq \lambda \Longrightarrow \operatorname{Var}\left(Y_{n: n}\right) \leq \operatorname{Var}\left(X_{n: n}\right)
$$

where $\lambda^{*}$ is given in (2.3.6).

Remark: In the special case of $\bar{F}(x)=e^{-x}$, Khaledi and Kochar [27] gives a lower bound of the variance of $X_{n: n}$ in terms of geometric mean, i.e.,

$$
\operatorname{Var}\left(X_{n: n}\right) \geq \frac{1}{\tilde{\lambda}} \sum_{i=1}^{n} \frac{1}{i^{2}} .
$$

Since the right spread order implies the variance being ordered (cf. Shaked and Shanthikumar [60]), Corollary 2.3.8 provides a better lower bound for the variance,

$$
\operatorname{Var}\left(X_{n: n}\right) \geq \frac{1}{\lambda^{*}} \sum_{i=1}^{n} \frac{1}{i^{2}}
$$

as $\lambda^{*} \leq \tilde{\lambda}$

\subsection{Star order}

Note that Theorem 2.3.1 does not have any restriction on the parameters, but restricted to the exponential random variables. In this section, I will discuss the PHR models. 
The following lemma is useful to prove the mail result.

Lemma 2.4.1 Let $\phi$ be a differentiable star-shaped function on $[0, \infty)$ such that $\phi(x) \geq x$ for all $x \geq 0$. Let $\psi$ be an increasing differentiable function such that

$$
x \frac{\psi^{\prime}(x)}{\psi(x)} \text { is incresing in } x .
$$

Then the function

$$
\psi \phi \psi^{-1}(x) \text { is also star-shaped in } x \text {. }
$$

Proof: Note that $\phi$ is star-shaped if and only if

$$
\frac{\phi(x)}{x} \text { is increasing in } x,
$$

which can be represented as

$$
\phi^{\prime}(x) \geq \frac{\phi(x)}{x}
$$

Hence, for the required result, it is sufficient to show

$$
\psi^{\prime} \phi \psi^{-1}(x) \frac{\phi^{\prime} \psi^{-1}(x)}{\psi^{\prime} \psi^{-1}(x)} \geq \frac{\psi \phi \psi^{-1}(x)}{x} .
$$

Using (2.4.1), the left side of (2.4.2) satisfies

$$
\frac{\psi^{\prime} \phi \psi^{-1}(x)}{\psi^{\prime} \psi^{-1}(x)} \phi^{\prime} \psi^{-1}(x) \geq \frac{\psi^{\prime} \phi \psi^{-1}(x)}{\psi^{\prime} \psi^{-1}(x)} \frac{\phi \psi^{-1}(x)}{\psi^{-1}(x)} .
$$

So, it is enough to prove

$$
\frac{\psi^{\prime} \phi \psi^{-1}(x)}{\psi^{\prime} \psi^{-1}(x)} \frac{\phi \psi^{-1}(x)}{\psi^{-1}(x)} \geq \frac{\psi \phi \psi^{-1}(x)}{x},
$$


i.e.,

$$
\frac{\psi^{\prime} \phi \psi^{-1}(x)}{\psi \phi \psi^{-1}(x)} \phi \psi^{-1}(x) \frac{1}{\psi^{-1}(x)} \geq \frac{\psi^{\prime} \psi^{-1}(x)}{x}
$$

as $\psi$ is increasing. Using the assumptions

$$
x \frac{\psi^{\prime}(x)}{\psi(x)} \text { is incresing in } x \text { and } \phi(x) \geq x,
$$

the required result follows immediately.

The following result compares the parallel systems according to the star order in the PHR models.

Theorem 2.4.2 Let $X_{1}, \ldots, X_{n}$ be independent random variables with $X_{i}$ having survival function $\bar{F}^{\lambda_{i}}, i=1, \ldots, n$, and let $Y_{1}, \ldots, Y_{n}$ be a random sample from a distribution with the common survival distribution $\bar{F}^{\lambda}$ where $\lambda \geq \tilde{\lambda}$. If

$$
\frac{R(x)}{x r(x)} \text { is increasing in } x \geq 0,
$$

then

$$
Y_{n: n} \leq_{\star} X_{n: n}
$$

where $R(x)=-\log \bar{F}(x)$ is the cumulative hazard rate function, and $r(x)=$ $f(x) / \bar{F}(x)$ is the hazard rate function of $F$.

Proof: Since $R(x)$ is increasing and

$$
R^{-1}(x)=\bar{F}^{-1}\left(e^{-x}\right),
$$


it holds that, for $x \geq 0, i=1, \ldots, n$,

$$
P\left(R\left(X_{i}\right)>x\right)=P\left(X_{i}>R^{-1}(x)\right)=\bar{F}^{\lambda_{i}}\left(\bar{F}^{-1}\left(e^{-x}\right)\right)=e^{-\lambda_{i} x} .
$$

So, making the transform

$$
X_{i}^{\prime}=R\left(X_{i}\right), \quad i=1, \cdots, n
$$

it follows that $X_{i}^{\prime}$ is exponential with hazard rate $\lambda_{i}$ for $i=1, \ldots, n$. Similarly, let $Y_{i}^{\prime}=H\left(Y_{i}\right)$ be exponential with hazard rate $\lambda$ for $i=1, \ldots, n$.

Observing that

$$
Y_{n: n}^{\prime} \stackrel{s t}{=} R\left(Y_{n: n}\right), \quad X_{n: n}^{\prime} \stackrel{s t}{=} R\left(X_{n: n}\right)
$$

it holds that

$$
\begin{gathered}
P\left(Y_{n: n} \leq x\right)=P\left(R^{-1}\left(Y_{n: n}^{\prime}\right) \leq x\right)=P\left(Y_{n: n}^{\prime} \leq R(x)\right)=G_{n: n}^{\prime}(R(x)), \\
P\left(X_{n: n} \leq x\right)=P\left(R^{-1}\left(X_{n: n}^{\prime}\right) \leq x\right)=P\left(X_{n: n}^{\prime} \leq R(x)\right)=F_{n: n}^{\prime}(R(x)),
\end{gathered}
$$

where $G_{n: n}^{\prime}(\cdot), F_{n: n}^{\prime}(\cdot)$ are distribution functions of $Y_{n: n}^{\prime}$ and $X_{n: n}^{\prime}$. Now, we need to prove

$$
R^{-1} F_{n: n}^{\prime-1} G_{n: n}^{\prime} R(x) \quad \text { is star-shaped. }
$$

From Theorem 2.3.1, $F_{n: n}^{\prime-1} G_{n: n}^{\prime}(x)$ is star-shaped on $[0, \infty)$.

Since $\lambda \geq \tilde{\lambda}$, by (2.1.7), it holds that,

$$
F_{n: n}^{\prime-1} G_{n: n}^{\prime}(x) \geq x
$$


By Lemma 2.4.1, it is enough to show

$$
x \frac{\left(R^{-1}(x)\right)^{\prime}}{R^{-1}(x)} \text { is increasing in } x,
$$

i.e.,

$$
\frac{R(x)}{x r(x)} \text { is increasing in } x
$$

which follows from the assumption.

\subsubsection{PHR models}

In this section, I will discuss the Weibull, Parato and Lomax distributions which belong to the PHR family, and for which Theorem 2.4.2 is applicable.

Proposition 2.4.3 Let $X_{1}, \ldots, X_{n}$ be independent Weibull random variables $W\left(\alpha, b_{i}\right)$.

Let $Y_{1}, \ldots, Y_{n}$ be a random sample of size $n$ from a weibull distribution $W(\alpha, b)$.

Then,

$$
Y_{n: n} \leq_{\star} X_{n: n}
$$

Proof: First, let us assume

$$
b=\tilde{b}=\left(\prod_{i=1}^{n} b_{i}\right)^{1 / n} .
$$

From Theorem 2.4.2, it is sufficient to prove

$$
\frac{R(x)}{x r(x)}=\frac{1}{\alpha} \quad \text { is increasing in } x \geq 0,
$$


which is obviously true. As the star transform order is scale invariant, the result follows immediately.

Using the similar argument in Proposition 2.3.3, the following result follows.

Proposition 2.4.4 Let $X_{1}, \ldots, X_{n}$ be independent Weibull random variables $W\left(\alpha, b_{i}\right)$. Let $Y_{1}, \ldots, Y_{n}$ be a random sample of size $n$ from a Weibull distribution $W(\alpha, b)$. Then,

$$
Y_{n: n} \leq_{d i s p} X_{n: n} \Longleftrightarrow Y_{n: n} \leq_{s t} X_{n: n}
$$

Remark: The above Proposition extends Corollary 3.1 in Khaledi and Kochar [31] by removing the constraint on $b$.

For Pareto distribution, observing that

$$
\frac{R(x)}{x r(x)}=\log (x / b)
$$

is increasing in $x$. Hence, we have the following result.

Proposition 2.4.5 Let $X_{1}, \ldots, X_{n}$ be independent Pareto random variables with shape parameter $\lambda_{i}$ for $i=1, \cdots, n$. Let $Y_{1}, \ldots, Y_{n}$ be a random sample of size $n$ from a Pareto distribution with $\lambda \geq \tilde{\lambda}$. Then,

$$
Y_{n: n} \leq_{*} X_{n: n} .
$$

For the Lomax distribution,

$$
\frac{R(x)}{x r(x)}=\frac{b+x}{x} \log \left(1+\frac{x}{b}\right)
$$


Taking the derivative with respect to $x$ and simplify, it holds that

$$
\frac{d}{d x} \frac{R(x)}{x r(x)}=\frac{1}{x}\left[1-\frac{b}{x} \log \left(1+\frac{x}{b}\right)\right] \geq 0, \quad x \geq 0, b>0 .
$$

Hence, we have the following Proposition.

Proposition 2.4.6 Let $X_{1}, \ldots, X_{n}$ be independent Lomax random variables with parameter $\lambda_{i}$ for $i=1, \cdots, n$. Let $Y_{1}, \ldots, Y_{n}$ be a random sample of size $n$ from a Lomax distribution with parameter $\lambda \geq \tilde{\lambda}$. Then,

$$
Y_{n: n} \leq_{*} X_{n: n}
$$

\subsection{Concluding remarks}

In this chapter, I have solved the open problem in Dykstra et al. [19] by proving the likelihood ratio order between the largest order statistics from heterogeneous samples and homogeneous samples. I have also obtained some new results on the skewness comparison between the largest order statistics. It provides equivalent characterizations of the dispersive order and the right spread order between two parallel systems. However, for the general case, the problem is still open. I make the conjecture that if $X_{1}, \ldots, X_{n}$ are independent exponential random variables with $X_{i}$ having hazard rate $\lambda_{i}, i=1, \ldots, n$, and $Y_{1}, \ldots, Y_{n}$ is a random sample of size $n$ from an exponential distribution with common hazard rate $\bar{\lambda}=\sum_{i=1}^{n} \lambda_{i} / n$, then

$$
Y_{k: n} \leq_{l r} X_{k: n}, k=1, \cdots, n
$$


The results of this Chapter are mainly based on Kochar and Xu [39] and Kochar and $X u[41]$. 


\section{Chapter 3}

\section{Spacings}

\subsection{Introduction}

Spacings are of great interest in many areas of statistics, in particular, in the characterizations of distributions, goodness-of-fit tests, auction theory, life testing and reliability models. A large number of goodness-of-fit tests are based on functions of sample spacings, see Balakrishnan and Rao [6].

Let $X_{1}, \ldots, X_{n}$ be $n$ nonnegative random variables. The random variables $D_{i: n}=X_{i: n}-X_{i-1: n}$ and $D_{i: n}^{*}=(n-i+1) D_{i: n}, i=1, \ldots, n$, with $X_{0: n} \equiv 0$, are respectively called simple spacings and normalized spacings. In the reliability context they correspond to times elapsed between successive failures of components in a system. In stochastic auction theory, $D_{n: n}$ and $D_{2: n}$ are of particular interest, which represent auction rents in buyer's auction and reverse auction in the second-price business auction (see $X u$ and $L i$ [64]). It is well known that the normalized spacings of a random sample from an exponential distribution are independent and identically distributed (i.i.d.) random variables having the same 
exponential distribution. Such a characterization may not hold for other distributions and much of the reliability theory deals with this aspect of spacings.

Pledger and Proschan [54] considered the problem of stochastically comparing the spacings of heterogeneous samples in the PHR model.

Theorem 3.1.1 If $X_{1}, \ldots, X_{n}$ are independent random variables with $X_{i}$ having survival function $\bar{F}(t)=e^{-\lambda_{i} R(t)}, i=1, \ldots, n$, where $R(t)$ is a concave and differentiable hazard function, then

$$
D_{i: n} \leq_{s t} D_{j: n} \text { for } \quad i \leq j,
$$

where $D_{i: n}=(n-i+1)\left(X_{i: n}-X_{i-1: n}\right)$ for $i=1, \cdots, n$ are the normalized spacings, and $X_{0: n} \equiv 0$.

Pledger and Proschan [54] also considered the problem of stochastically comparing the spacings of nonidentical independent exponential random variables with those corresponding to stochastically comparable independent and identically distributed exponential random variables.

Theorem 3.1.2 If $X_{1}, \ldots, X_{n}$ are independent exponential random variables with $X_{i}$ having hazard rate $\lambda_{i}, i=1, \ldots, n$, and $Y_{1}, \ldots, Y_{n}$ is a random sample of size $n$ from an exponential distribution with common hazard rate $\bar{\lambda}=\sum_{i=1}^{n} \lambda_{i} / n$, then

$$
D_{i: n}^{*} \leq_{s t} D_{i: n}
$$


where $D_{i: n}^{*}=(n-i+1)\left(Y_{i: n}-Y_{i-1: n}\right)$ for $i=1, \cdots, n$ are the normalized spacings from $Y_{i}$ 's, and $Y_{0: n} \equiv 0$.

Kochar and Korwar [34] strengthened and extended this result from stochastic ordering to likelihood ratio ordering and dispersive ordering.

Theorem 3.1.3 If $X_{1}, \ldots, X_{n}$ are independent exponential random variables with $X_{i}$ having hazard rate $\lambda_{i}, i=1, \ldots, n$, and $Y_{1}, \ldots, Y_{n}$ is a random sample of size $n$ from an exponential distribution with common hazard rate $\bar{\lambda}=\sum_{i=1}^{n} \lambda_{i} / n$, then

$$
D_{i: n}^{*} \leq_{l r} D_{i: n} \quad \text { and } \quad D_{i: n}^{*} \leq_{d i s p} D_{i: n}, \quad i=1, \ldots, n .
$$

Kochar and Rojo [36] further strengthened Theorem 3.1.3 to multivariate likelihood ratio order.

Theorem 3.1.4 If $X_{1}, \ldots, X_{n}$ are independent exponential random variables with $X_{i}$ having hazard rate $\lambda_{i}, i=1, \ldots, n$, and $Y_{1}, \ldots, Y_{n}$ is a random sample of size $n$ from an exponential distribution with common hazard rate $\bar{\lambda}=\sum_{i=1}^{n} \lambda_{i} / n$, then,

$$
\left(D_{1: n}^{*}, \ldots, D_{n: n}^{*}\right) \leq_{l r}\left(D_{1: n}, \ldots, D_{n: n}\right)
$$

\subsection{Equivalent characterizations}

Let $X_{1}, \ldots, X_{n}$ be independent exponential random variables with $X_{i}$ having hazard rate $\lambda_{i}, i=1, \ldots, n$, and $Y_{1}, \ldots, Y_{n}$ be a random sample of size $n$ from an 
exponential distribution with common hazard rate $\lambda$. One natural question is to find sufficient and necessary conditions for comparing simple sample spacings of $D_{k: n}$ from $X_{i}$ 's and $H_{k: n}$ from $Y_{i}$ 's. Kochar and Korwar [34] proved that the distribution function of $D_{k: n}^{*}$ for $i=2, \cdots, n$ is a mixture of independent exponential random variables with the density function

$$
f_{D_{k: n}^{*}}(x)=\sum_{\mathbf{r}} \frac{\prod_{i=1}^{n} \lambda_{i}}{\prod_{i=1}^{n} \sum_{j=i}^{n} \lambda_{r_{j}}}\left(\frac{\sum_{j=k}^{n} \lambda_{r_{j}}}{n-i+1}\right) \exp \left\{\frac{-x \sum_{j=k}^{n} \lambda_{r_{j}}}{n-i+1}\right\},
$$

where $\mathbf{r}$ extends over all of the permutations of $\{1,2, \cdots, n\}$. Hence, the distribution of $D_{k: n}$ could be represented as

$$
F_{D_{k: n}}(x)=\sum_{j \in \mathbf{r}} p_{j} F_{\lambda_{k j}^{*}}(x),
$$

where $j$ denotes a permutation of $\left(\lambda_{1}, \ldots, \lambda_{n}\right)$ belonging to $\mathbf{r}$ and

$$
p_{j}=\frac{\prod_{i=1}^{n} \lambda_{i}}{\prod_{i=1}^{n} \sum_{j=i}^{n} \lambda_{r_{j}}},
$$

and

$$
\lambda_{k j}^{*}=\frac{\sum_{j=k}^{n} \lambda_{r_{j}}}{(n-k+1)^{2}},
$$

and $F_{\lambda_{k j}^{*}}$ means an exponential distribution with hazard rate $\lambda_{k j}^{*}$.

Note that, the distribution function of $H_{k: n}$ is

$$
F_{H_{k: n}}(x)=F_{\lambda /(n-k+1)}(x)=1-\exp \left\{-\frac{\lambda x}{n-k+1}\right\}
$$

The following results provide sufficient and necessary conditions for stochastically comparing $D_{k: n}$ and $H_{k: n}$. 
Theorem 3.2.1 Let $X_{1}, \ldots, X_{n}$ be independent exponential random variables with $X_{i}$ having hazard rate $\lambda_{i}, i=1, \ldots, n$, and $Y_{1}, \ldots, Y_{n}$ be a random sample of size $n$ from an exponential distribution with common hazard rate $\lambda$. Then, for $k \geq 2$,

(a) $H_{k: n} \leq_{l r} D_{k: n}$ or

(b) $H_{k: n} \leq_{r h} D_{k: n}$;

if and only if

$$
\lambda \geq \frac{1}{n-k+1} \frac{\sum_{\mathbf{r}} \frac{\prod_{i=1}^{n} \lambda_{i}}{\prod_{i=1}^{n} \sum_{j=i}^{n} \lambda_{r_{j}}}\left(\sum_{j=k}^{n} \lambda_{r_{j}}\right)^{2}}{\sum_{\mathbf{r}} \frac{\prod_{i=1}^{n} \lambda_{i}}{\prod_{i=1}^{n} \sum_{j=i}^{n} \lambda_{r_{j}}} \sum_{j=k}^{n} \lambda_{r_{j}}} .
$$

Proof: The necessary and sufficient condition for likelihood ration order between $H_{k: n}$ and $D_{k: n}$ follows from Lemma 3.1 of Zhao et al. [66]. It remains to prove that the condition on $\lambda$ is a necessary condition for the reverse hazard rate order.

Note that the reverse hazard rate of $D_{k: n}$ for $k \geq 2$, by Taylor's expansion around zero, is

$$
\begin{aligned}
\tilde{r}_{D_{k: n}}(x) & =\frac{\sum_{j \in \mathbf{r}} p_{j} \lambda_{k j}^{*} \exp \left\{-\lambda_{k j}^{*} x\right\}}{\sum_{j \in \mathbf{r}} p_{j}\left(1-\exp \left\{-\lambda_{k j}^{*} x\right\}\right)} \\
& =\frac{\sum_{j \in \mathbf{r}} p_{j} \lambda_{k j}^{*}\left(1-\lambda_{k j}^{*} x+o(x)\right)}{\sum_{j \in \mathbf{r}} p_{j} \lambda_{k j}^{*} x+o(x)} \\
& =\frac{\sum_{j \in \mathbf{r}} p_{j} \lambda_{k j}^{*}-\sum_{j \in \mathbf{r}} p_{j}\left(\lambda_{k j}^{*}\right)^{2} x+o(x)}{\sum_{j \in \mathbf{r}} p_{j} \lambda_{k j}^{*} x+o(x)} .
\end{aligned}
$$

Similarly, for $i \geq 2$,

$$
\tilde{r}_{H_{k: n}}(x)=\frac{\lambda-\lambda^{2} x /(n-k+1)+o(x)}{\lambda x+o(x)} .
$$


Then

$$
\tilde{r}_{H_{k: n}}(x) \leq \tilde{r}_{D_{k: n}}(x)
$$

implies

$$
\lambda \geq(n-k+1) \frac{\sum_{j \in \mathbf{r}} p_{j}\left(\lambda_{k j}^{*}\right)^{2}}{\sum_{j \in \mathbf{r}} p_{j} \lambda_{k j}^{*}} .
$$

The result follows immediately.

Analogously, we have an equivalent condition for hazard rate order, dispersive order and stochastic order.

Theorem 3.2.2 Let $X_{1}, \ldots, X_{n}$ be independent exponential random variables with $X_{i}$ having hazard rate $\lambda_{i}, i=1, \ldots, n$, and $Y_{1}, \ldots, Y_{n}$ be a random sample of size $n$ from an exponential distribution with common hazard rate $\lambda$. Then, for $k \geq 2$,

(a) $H_{k: n} \leq_{h r} D_{k: n}$ or

(b) $H_{k: n} \leq_{d i s p} D_{k: n}$ or

(c) $H_{k: n} \leq_{s t} D_{k: n}$

if and only if

$$
\lambda \geq \frac{1}{n-k+1} \sum_{\mathbf{r}} \frac{\prod_{i=1}^{n} \lambda_{i}}{\prod_{i=1}^{n} \sum_{j=i}^{n} \lambda_{r_{j}}} \sum_{j=k}^{n} \lambda_{r_{j}} .
$$

Proof: Using Lemma 2.1 in Păltănea [53], it is easy to show the condition on $\lambda$ is necessary and sufficient condition for hazard rate order. Since $H_{k: n}$ is an exponential random variable, according to Theorem 3.B.20 of Shaked and Shanthikumar [60], (a) implies (b). By Theorem 3.B.13 there, (b) implies (c). Hence, 
it is enough to show the condition on $\lambda$ is the necessary condition for the stochastic order. Using Taylor's expansion around zero for the distribution function, for $k \geq 2$,

$$
F_{D_{k: n}}(x)=\sum_{j \in \mathbf{r}} p_{j}\left(\lambda_{k j}^{*} x+o(x)\right)
$$

and

$$
F_{H_{k: n}}(x)=\frac{\lambda}{n-k+1} x+o(x)
$$

So,

$$
F_{H_{k: n}}(x) \geq F_{D_{k: n}}(x)
$$

implies,

$$
\lambda \geq(n-k+1) \sum_{j \in \mathbf{r}} p_{j} \lambda_{k j}^{*} .
$$

Hence, the required result follows.

Theorem 3.2.3 Let $X_{1}, \ldots, X_{n}$ be independent exponential random variables with $X_{i}$ having hazard rate $\lambda_{i}, i=1, \ldots, n$, and $Y_{1}, \ldots, Y_{n}$ be a random sample of size $n$ from an exponential distribution with common hazard rate $\lambda$. Then, for $k \geq 2$,

(a) $H_{k: n} \leq_{m r l} D_{k: n}$ or

(b) $H_{k: n} \leq_{R S} D_{k: n}$ or

(c) $\mathrm{E} H_{k: n} \leq \mathrm{E} D_{k: n}$

if and only if

$$
\lambda \geq \frac{1}{n-k+1} \frac{1}{\sum_{\mathbf{r}} \frac{\prod_{i=1}^{n} \lambda_{i}}{\sum_{j=k}^{n} \lambda_{r_{j}} \prod_{i=1}^{n} \sum_{j=i}^{n} \lambda_{r_{j}}}} .
$$


Proof: From Lemma 2.6 of Zhao and Balakrishnan [68], it is seen that the condition on $\lambda$ is necessary and sufficient for the mean residual life order. By Theorem 3.C.6 of Shaked and Shanthikumar [60], (a) implies (b). It is easy to see that (b) implies (c). Hence, it is enough to show the condition on $\lambda$ is a necessary condition for (c). Note that

$$
\mathrm{E}\left(D_{k: n}\right)=\sum_{\mathbf{r}} \frac{p_{j}}{\lambda_{k j}^{*}}
$$

and

$$
\mathrm{E}\left(H_{k: n}\right)=\frac{n-k+1}{\lambda}
$$

the result follows immediately.

Example 3.2.4 Assume $X_{1}, X_{2}, X_{3}$ are exponential random variables with parameters $\lambda_{1}, \lambda_{2}, \lambda_{3}$, and $Y_{1}, Y_{2}, Y_{3}$ are exponential random variables from the same distribution with parameter $\lambda$. Then, from Theorem 3.2.2,

$$
Y_{3: 3}-Y_{2: 3} \leq_{h r} X_{3: 3}-X_{2: 3}
$$

if and only if

$$
\lambda \geq \lambda_{h r}=\frac{2 \lambda_{1} \lambda_{2} \lambda_{3}}{\lambda_{1}+\lambda_{2}+\lambda_{3}}\left(\frac{1}{\lambda_{2}+\lambda_{3}}+\frac{1}{\lambda_{1}+\lambda_{3}}+\frac{1}{\lambda_{1}+\lambda_{2}}\right) .
$$

From Theorem 3.2.1,

$$
Y_{3: 3}-Y_{2: 3} \leq_{l r} X_{3: 3}-X_{2: 3}
$$


if and only if

$$
\lambda \geq \lambda_{l r}=\frac{3}{2}\left(\frac{1}{\lambda_{2}+\lambda_{3}}+\frac{1}{\lambda_{1}+\lambda_{3}}+\frac{1}{\lambda_{1}+\lambda_{2}}\right)^{-1}
$$

From Theorem 3.2.3

$$
Y_{3: 3}-Y_{2: 3} \leq_{R S} X_{3: 3}-X_{2: 3}
$$

if and only if

$\lambda \geq \lambda_{R S}=\left[\frac{\lambda_{1} \lambda_{2} \lambda_{3}}{\lambda_{1}+\lambda_{2}+\lambda_{3}}\left(\frac{\lambda_{2}^{2}+\lambda_{3}^{2}}{\left(\lambda_{2}+\lambda_{3}\right) \lambda_{2}^{2} \lambda_{3}^{2}}+\frac{\lambda_{1}^{2}+\lambda_{3}^{2}}{\left(\lambda_{1}+\lambda_{3}\right) \lambda_{1}^{2} \lambda_{3}^{2}}+\frac{\lambda_{2}^{2}+\lambda_{1}^{2}}{\left(\lambda_{2}+\lambda_{1}\right) \lambda_{2}^{2} \lambda_{1}^{2}}\right)\right]^{-1}$.

It is worth noting that

$$
\lambda_{l r} \geq \lambda_{h r} \geq \lambda_{R S}
$$

For example, let

$$
\lambda_{1}=1, \lambda_{2}=2, \lambda_{3}=3
$$

Then,

$$
\lambda_{l r}=1.91489>\lambda_{h r}=1.56667>\lambda_{R S}=1.30435 .
$$

\subsection{Range}

Sample range is one of the criteria for comparing variabilities among distributions and hence it is important to study its stochastic properties.

Kochar and Rojo [36] proved the following result. 
Theorem 3.3.1 If $X_{1}, \ldots, X_{n}$ are independent exponential random variables with $X_{i}$ having hazard rate $\lambda_{i}, i=1, \ldots, n$, and $Y_{1}, \ldots, Y_{n}$ is a random sample of size $n$ from an exponential distribution with common hazard rate $\bar{\lambda}=\sum_{i=1}^{n} \lambda_{i} / n$, then,

$$
Y_{n: n}-Y_{1: n} \leq_{s t} X_{n: n}-X_{1: n}
$$

Khaledi and Kochar [28] improved upon this result for sample range.

Theorem 3.3.2 If $X_{1}, \ldots, X_{n}$ are independent exponential random variables with $X_{i}$ having hazard rate $\lambda_{i}, i=1, \ldots, n$, and $Y_{1}, \ldots, Y_{n}$ is a random sample of size $n$ from an exponential distribution with common hazard rate $\tilde{\lambda}=\left(\prod_{i=1}^{n} \lambda_{i}\right)^{1 / n}$, then,

$$
Y_{n: n}-Y_{1: n} \leq_{s t} X_{n: n}-X_{1: n}
$$

Recently, Zhao and Li [69] obtained the necessary and sufficient condition for comparing sample ranges from heterogeneous and homogeneous exponential samples.

Theorem 3.3.3 If $X_{1}, \ldots, X_{n}$ are independent exponential random variables with $X_{i}$ having hazard rate $\lambda_{i}, i=1, \ldots, n$, and $Y_{1}, \ldots, Y_{n}$ is a random sample of size $n$ from an exponential distribution with common hazard rate $\lambda$, then,

$$
Y_{n: n}-Y_{1: n} \leq_{s t} X_{n: n}-X_{1: n} \Longleftrightarrow \lambda \geq \hat{\lambda}=\left(\frac{\prod_{i=1}^{n} \lambda_{i}}{\bar{\lambda}}\right)^{1 /(n-1)},
$$

where $\bar{\lambda}=\sum_{i=1}^{n} \lambda_{i} / n$.

It is remarkable that $\hat{\lambda} \leq \tilde{\lambda}$, which improves upon the results of Theorem 3.3.2. 


\subsubsection{Reversed hazard rate order}

In this section, I prove the reversed hazard rate order between the ranges from heterogeneous exponential samples and homogeneous exponential samples.

Theorem 3.3.4 Let $X_{1}, \ldots, X_{n}$ be independent exponential random variables with $X_{i}$ having hazard rate $\lambda_{i}, i=1, \ldots, n$. Let $Y_{1}, \ldots, Y_{n}$ be a random sample of size $n$ from an exponential distribution with common hazard rate $\bar{\lambda}=\sum_{i=1}^{n} \lambda_{i} / n$. Then

$$
Y_{n: n}-Y_{1: n} \leq_{r h} X_{n: n}-X_{1: n}
$$

Proof: Denote by $R_{X}=X_{n: n}-X_{1: n}$ and $R_{Y}=Y_{n: n}-Y_{1: n}$ the sample ranges of $X_{i}$ 's and $Y_{i}^{\prime}$ 's, respectively. From David and Nagaraja ([15], p. 26) the distribution function of $R_{X}$ is, for $x \geq 0$,

$$
F_{R_{X}}(x)=\frac{1}{\sum_{i=1}^{n} \lambda_{i}} \sum_{i=1}^{n} \lambda_{i} \prod_{j=1, j \neq i}^{n}\left(1-e^{-\lambda_{j} x}\right) .
$$


Thus, we have the density function of $R_{X}$ as, for $x \geq 0$,

$$
\begin{aligned}
f_{R_{X}}(x) & =\frac{1}{\sum_{i=1}^{n} \lambda_{i}}\left(\sum_{i=1}^{n} \lambda_{i} \prod_{j=1, j \neq i}^{n}\left(1-e^{-\lambda_{j} x}\right)\right)^{\prime} \\
& =\frac{1}{\sum_{i=1}^{n} \lambda_{i}}\left[\sum_{i=1}^{n} \lambda_{i} \sum_{j=1, j \neq i}^{n} \lambda_{j} e^{-\lambda_{j} x} \prod_{k=1, k \neq i, j}^{n}\left(1-e^{-\lambda_{k} x}\right)\right] \\
& =\frac{1}{\sum_{i=1}^{n} \lambda_{i}}\left[\sum_{i=1}^{n} \lambda_{i} \sum_{j=1, j \neq i}^{n} \frac{\lambda_{j} e^{-\lambda_{j} x}}{\left(1-e^{-\lambda_{i} x}\right)\left(1-e^{-\lambda_{j} x}\right)}\right] \prod_{i=1}^{n}\left(1-e^{-\lambda_{i} x}\right) \\
& =\frac{\prod_{i=1}^{n}\left(1-e^{-\lambda_{i} x}\right)}{\sum_{i=1}^{n} \lambda_{i}} \sum_{i=1}^{n} \frac{\lambda_{i}}{1-e^{-\lambda_{i} x}} \sum_{j=1, j \neq i}^{n} \frac{\lambda_{j} e^{-\lambda_{j} x}}{1-e^{-\lambda_{j} x}} .
\end{aligned}
$$

Hence, the reversed hazard rate of $R_{X}$ is, for $x \geq 0$,

$$
\tilde{r}_{R_{X}}(x)=\frac{\sum_{i=1}^{n} \frac{\lambda_{i}}{1-e^{-\lambda_{i} x}} \sum_{j=1, j \neq i}^{n} \frac{\lambda_{j} e^{-\lambda_{j} x}}{1-e^{-\lambda_{j} x}}}{\sum_{i=1}^{n} \frac{\lambda_{i}}{1-e^{-\lambda_{i} x}}} .
$$

The reversed hazard rate of $R_{Y}$ is, for $x \geq 0$,

$$
\tilde{r}_{R_{Y}}(x)=(n-1) \frac{\bar{\lambda} e^{-\bar{\lambda} x}}{1-e^{-\bar{\lambda} x}} .
$$

Since, for $x \geq 0$,

$$
\sum_{i=1}^{n} \frac{\lambda_{i}}{1-e^{-\lambda_{i} x}} \geq n \sqrt[n]{\prod_{i=1}^{n} \frac{\lambda_{i}}{1-e^{-\lambda_{i} x}}}
$$

and

$$
\sum_{i=1}^{n} \frac{1-e^{-\lambda_{i} x}}{\lambda_{i}} \geq n \sqrt[n]{\prod_{i=1}^{n} \frac{1-e^{-\lambda_{i} x}}{\lambda_{i}}}
$$


it holds that,

$$
\sum_{i=1}^{n} \frac{\lambda_{i}}{1-e^{-\lambda_{i} x}} \sum_{i=1}^{n} \frac{1-e^{-\lambda_{i} x}}{\lambda_{i}} \geq n^{2} .
$$

Note that, from inequality (2.2.2),

$$
\sum_{i=1}^{n} \frac{\lambda_{i}^{2} e^{-\lambda_{i} x}}{\left(1-e^{-\lambda_{i} x}\right)^{2}} \sum_{i=1}^{n} \frac{1-e^{-\lambda_{i} x}}{\lambda_{i}} \leq n \sum_{i=1}^{n} \frac{\lambda_{i} e^{-\lambda_{i} x}}{1-e^{-\lambda_{i} x}} .
$$

Combining this with inequality (3.3.2), we get for $x \geq 0$,

$$
\sum_{i=1}^{n} \frac{\lambda_{i}^{2} e^{-\lambda_{i} x}}{\left(1-e^{-\lambda_{i} x}\right)^{2}} \leq \frac{1}{n} \sum_{i=1}^{n} \frac{\lambda_{i}}{1-e^{-\lambda_{i} x}} \sum_{i=1}^{n} \frac{\lambda_{i} e^{-\lambda_{i} x}}{1-e^{-\lambda_{i} x}} .
$$

That is, for $x \geq 0$,

$$
\begin{aligned}
& \sum_{i=1}^{n} \frac{\lambda_{i}}{1-e^{-\lambda_{i} x}} \sum_{i=1}^{n} \frac{\lambda_{i} e^{-\lambda_{i} x}}{1-e^{-\lambda_{i} x}}-\sum_{i=1}^{n} \frac{\lambda_{i}^{2} e^{-\lambda_{i} x}}{\left(1-e^{-\lambda_{i} x}\right)^{2}} \\
& \geq \frac{n-1}{n} \sum_{i=1}^{n} \frac{\lambda_{i}}{1-e^{-\lambda_{i} x}} \sum_{i=1}^{n} \frac{\lambda_{i} e^{-\lambda_{i} x}}{1-e^{-\lambda_{i} x}} .
\end{aligned}
$$

Observing that,

$$
f(x)=\frac{x e^{-x}}{1-e^{-x}}
$$

is convex in $x \geq 0$. It follows from Jensen's inequality that

$$
\frac{1}{n} \sum_{i=1}^{n} \frac{\lambda_{i} x e^{-\lambda_{i} x}}{1-e^{-\lambda_{i} x}} \geq \frac{\bar{\lambda} x e^{-\bar{\lambda} x}}{1-e^{-\bar{\lambda} x}}
$$

i.e,

$$
\frac{1}{n} \sum_{i=1}^{n} \frac{\lambda_{i} e^{-\lambda_{i} x}}{1-e^{-\lambda_{i} x}} \geq \frac{\bar{\lambda} e^{-\bar{\lambda} x}}{1-e^{-\bar{\lambda} x}}
$$

Using inequalities (3.3.3) and (3.3.4), it holds that, for $x \geq 0$,

$\sum_{i=1}^{n} \frac{\lambda_{i}}{1-e^{-\lambda_{i} x}} \sum_{i=1}^{n} \frac{\lambda_{i} e^{-\lambda_{i} x}}{1-e^{-\lambda_{i} x}}-\sum_{i=1}^{n} \frac{\lambda_{i}^{2} e^{-\lambda_{i} x}}{\left(1-e^{-\lambda_{i} x}\right)^{2}} \geq(n-1) \frac{\bar{\lambda} e^{-\bar{\lambda} x}}{1-e^{-\bar{\lambda} x}} \sum_{i=1}^{n} \frac{\lambda_{i}}{1-e^{-\lambda_{i} x}}$. 
Hence, for $x \geq 0$,

$$
\frac{\sum_{i=1}^{n} \frac{\lambda_{i}}{1-e^{-\lambda_{i} x}} \sum_{j=1, j \neq i}^{n} \frac{\lambda_{j} e^{-\lambda_{j} x}}{1-e^{-\lambda_{j} x}}}{\sum_{i=1}^{n} \frac{\lambda_{i}}{1-e^{-\lambda_{i} x}}} \geq(n-1) \frac{\bar{\lambda} e^{-\bar{\lambda} x}}{1-e^{-\bar{\lambda} x}},
$$

i.e.,

$$
\tilde{r}_{R_{X}}(x) \geq \tilde{r}_{R_{Y}}(x) .
$$

The required result follows immediately.

\subsubsection{Likelihood ratio order}

In this section, the likelihood ratio order between the ranges is shown to be true.

The following two inequalities are used to prove the main result.

Lemma 3.3.5 For $\lambda_{i}>0, i=1, \cdots, n$,

$$
\sum_{i=1}^{n} \frac{\lambda_{i}}{1-e^{-\lambda_{i}}} \sum_{j=1, j \neq i}^{n} \frac{\lambda_{j} e^{-\lambda_{j}}}{1-e^{-\lambda_{j}}} \geq \frac{n-1}{n}\left(\sum_{i=1}^{n} \frac{\lambda_{i}}{1-e^{-\lambda_{i}}}\right)\left(\sum_{i=1}^{n} \frac{\lambda_{i} e^{-\lambda_{i}}}{1-e^{-\lambda_{i}}}\right) .
$$

Proof: Note that, the above inequality could be written as,

$$
\begin{aligned}
& \left(\sum_{i=1}^{n} \frac{\lambda_{i}}{1-e^{-\lambda_{i}}}\right)\left(\sum_{i=1}^{n} \frac{\lambda_{i} e^{-\lambda_{i}}}{1-e^{-\lambda_{i}}}\right)-\sum_{i=1}^{n} \frac{\lambda_{i}^{2} e^{-\lambda_{i}}}{\left(1-e^{-\lambda_{i}}\right)^{2}} \\
& \geq \frac{n-1}{n}\left(\sum_{i=1}^{n} \frac{\lambda_{i}}{1-e^{-\lambda_{i}}}\right)\left(\sum_{i=1}^{n} \frac{\lambda_{i} e^{-\lambda_{i}}}{1-e^{-\lambda_{i}}}\right) .
\end{aligned}
$$

That is,

$$
\sum_{i=1}^{n} \frac{\lambda_{i}^{2} e^{-\lambda_{i}}}{\left(1-e^{-\lambda_{i}}\right)^{2}} \leq \frac{1}{n}\left(\sum_{i=1}^{n} \frac{\lambda_{i}}{1-e^{-\lambda_{i}}}\right)\left(\sum_{i=1}^{n} \frac{\lambda_{i} e^{-\lambda_{i}}}{1-e^{-\lambda_{i}}}\right)
$$


By Lemma 2.2.1,

$$
\frac{\lambda_{i}}{1-e^{-\lambda_{i}}} \quad \text { is increasing in } \lambda_{i}>0
$$

and, it is easy to verify that,

$$
\frac{\lambda_{i} e^{-\lambda_{i}}}{1-e^{-\lambda_{i}}} \quad \text { is decreasing in } \lambda_{i}>0 \text {. }
$$

Thus, the required result follows from Chebyshev's sum inequality (cf. Mitrinović [49], Theorem 1, p.36).

Lemma 3.3.6 For $\lambda_{i}>0, i=1, \cdots, n$,

$$
\frac{\bar{\lambda}}{1-e^{-\bar{\lambda}}} \geq \frac{n}{\sum_{i=1}^{n} \frac{1-e^{-\lambda_{i}}}{\lambda_{i}}} .
$$

Proof: Observing that

$$
f(x)=\frac{1-e^{-x}}{x}
$$

is convex in $x>0$, the result follows immediately from Jensen's inequality.

The following lemma will also be used to prove our main result.

Lemma 3.3.7 For $\lambda_{i}>0, i=1, \cdots, n$,

$$
\frac{\sum_{i=1}^{n} \frac{\lambda_{i}}{1-e^{-\lambda_{i}}} \sum_{j=1, j \neq i}^{n} \frac{\lambda_{j}^{2} e^{-\lambda_{j}}}{\left(1-e^{-\lambda_{j}}\right)^{2}}}{\sum_{i=1}^{n} \frac{\lambda_{i}}{1-e^{-\lambda_{i}}} \sum_{j=1, j \neq i}^{n} \frac{\lambda_{j} e^{-\lambda_{j}}}{1-e^{-\lambda_{j}}}} \leq \frac{\sum_{j=1}^{n} \frac{\lambda_{j}^{2} e^{-\lambda_{j}}}{\left(1-e^{-\lambda_{j}}\right)^{2}}}{\sum_{j=1}^{n} \frac{\lambda_{j} e^{-\lambda_{j}}}{1-e^{-\lambda_{j}}}} .
$$


Proof: Note that, inequality (3.3.6) is equivalent to,

$$
\begin{aligned}
& \sum_{i=1}^{n} \frac{\lambda_{i}}{1-e^{-\lambda_{i} x}} \sum_{j=1, j \neq i}^{n} \frac{\lambda_{j}^{2} e^{-\lambda_{j}}}{\left(1-e^{-\lambda_{j}}\right)^{2}} \times \sum_{j=1}^{n} \frac{\lambda_{j} e^{-\lambda_{j}}}{1-e^{-\lambda_{j}}} \\
\leq & \sum_{i=1}^{n} \frac{\lambda_{i}}{1-e^{-\lambda_{i}}} \sum_{j=1, j \neq i}^{n} \frac{\lambda_{j} e^{-\lambda_{j}}}{1-e^{-\lambda_{j}}} \times \sum_{j=1}^{n} \frac{\lambda_{j}^{2} e^{-\lambda_{j}}}{\left(1-e^{-\lambda_{j}}\right)^{2}} .
\end{aligned}
$$

That is,

$$
\begin{aligned}
& \left(\sum_{i=1}^{n} \frac{\lambda_{i}}{1-e^{-\lambda_{i}}}\right)\left(\sum_{j=1}^{n} \frac{\lambda_{j}^{2} e^{-\lambda_{j}}}{\left(1-e^{-\lambda_{j}}\right)^{2}}\right)\left(\sum_{j=1}^{n} \frac{\lambda_{j} e^{-\lambda_{j}}}{1-e^{-\lambda_{j}}}\right) \\
& -\left(\sum_{i=1}^{n} \frac{\lambda_{i}^{3} e^{-\lambda_{i}}}{\left(1-e^{-\lambda_{i}}\right)^{3}}\right)\left(\sum_{j=1}^{n} \frac{\lambda_{j} e^{-\lambda_{j}}}{1-e^{-\lambda_{j}}}\right) \\
\leq & \left(\sum_{i=1}^{n} \frac{\lambda_{i}}{1-e^{-\lambda_{i}}}\right)\left(\sum_{j=1}^{n} \frac{\lambda_{j} e^{-\lambda_{j}}}{1-e^{-\lambda_{j}}}\right)\left(\sum_{j=1}^{n} \frac{\lambda_{j}^{2} e^{-\lambda_{j}}}{\left(1-e^{-\lambda_{j}}\right)^{2}}\right)-\left[\sum_{j=1}^{n} \frac{\lambda_{j}^{2} e^{-\lambda_{j}}}{\left(1-e^{-\lambda_{j}}\right)^{2}}\right]^{2} .
\end{aligned}
$$

Hence, it is sufficient to prove that, for $\lambda_{i}>0, i=1, \cdots, n$,

$$
\left[\sum_{i=1}^{n} \frac{\lambda_{i}^{2} e^{-\lambda_{i}}}{\left(1-e^{-\lambda_{i}}\right)^{2}}\right]^{2} \leq \sum_{i=1}^{n} \frac{\lambda_{i}^{3} e^{-\lambda_{i}}}{\left(1-e^{-\lambda_{i}}\right)^{3}} \sum_{i=1}^{n} \frac{\lambda_{i} e^{-\lambda_{i}}}{1-e^{-\lambda_{i}}},
$$

which follows directly from Cauchy-Schwarz inequality (cf. Mitrinović [49], Theorem 1, p.30).

The following theorem states that the ratio of the reversed hazard rates is monotone, which may be of independent interest.

Theorem 3.3.8 For $x \geq 0$,

$$
\frac{\tilde{r}_{R_{X}}(x)}{\tilde{r}_{R_{Y}}(x)} \text { is increasing, }
$$

where $\tilde{r}_{R_{X}}(x)$ and $\tilde{r}_{R_{Y}}(x)$ are reversed hazard rates of $R_{X}=X_{n: n}-X_{1: n}$ and $R_{Y}=Y_{n: n}-Y_{1: n}$, respectively. 
Proof: Since the reversed hazard rate of $R_{X}$ is, for $x \geq 0$,

$$
\tilde{r}_{R_{X}}(x)=\frac{\sum_{i=1}^{n} \frac{\lambda_{i}}{1-e^{-\lambda_{i} x}} \sum_{j=1, j \neq i}^{n} \frac{\lambda_{j} e^{-\lambda_{j} x}}{1-e^{-\lambda_{j} x}}}{\sum_{i=1}^{n} \frac{\lambda_{i}}{1-e^{-\lambda_{i} x}}} .
$$

The reversed hazard rate of $R_{Y}$ is, for $x \geq 0$,

$$
\tilde{r}_{R_{Y}}(x)=(n-1) \frac{\bar{\lambda} e^{-\bar{\lambda} x}}{1-e^{-\bar{\lambda} x}} .
$$

Hence, it is enough to prove that,

$$
\frac{\sum_{i=1}^{n} \frac{\lambda_{i}}{1-e^{-\lambda_{i} x}} \sum_{j=1, j \neq i}^{n} \frac{\lambda_{j} e^{-\lambda_{j} x}}{1-e^{-\lambda_{j} x}}}{\frac{\bar{\lambda} e^{-\bar{\lambda} x}}{1-e^{-\bar{\lambda} x}} \sum_{i=1}^{n} \frac{\lambda_{i}}{1-e^{-\lambda_{i} x}}}
$$

is increasing in $x \geq 0$. Differentiating with respect to $x$, it is equivalent to proving that,

$$
\begin{aligned}
& {\left[\sum_{i=1}^{n} \frac{\lambda_{i}^{2} e^{-\lambda_{i} x}}{\left(1-e^{-\lambda_{i} x}\right)^{2}} \sum_{j=1, j \neq i}^{n} \frac{\lambda_{j} e^{-\lambda_{j} x}}{1-e^{-\lambda_{j} x}}+\sum_{i=1}^{n} \frac{\lambda_{i}}{1-e^{-\lambda_{i} x}} \sum_{j=1, j \neq i}^{n} \frac{\lambda_{j}^{2} e^{-\lambda_{j} x}}{\left(1-e^{-\lambda_{j} x}\right)^{2}}\right]} \\
& \quad \times\left(\frac{\bar{\lambda} e^{-\bar{\lambda} x}}{1-e^{-\bar{\lambda} x}} \sum_{i=1}^{n} \frac{\lambda_{i}}{1-e^{-\lambda_{i} x}}\right) \\
& \leq\left[\frac{\bar{\lambda}^{2} e^{-\bar{\lambda} x}}{\left(1-e^{-\bar{\lambda} x}\right)^{2}} \sum_{i=1}^{n} \frac{\lambda_{i}}{1-e^{-\lambda_{i} x}}+\frac{\bar{\lambda} e^{-\bar{\lambda} x}}{1-e^{-\bar{\lambda} x}} \sum_{i=1}^{n} \frac{\lambda_{i}^{2} e^{-\lambda_{i} x}}{\left(1-e^{-\lambda_{i} x}\right)^{2}}\right] \\
& \quad \times\left(\sum_{i=1}^{n} \frac{\lambda_{i}}{1-e^{-\lambda_{i} x}} \sum_{j=1, j \neq i}^{n} \frac{\lambda_{j} e^{-\lambda_{j} x}}{1-e^{-\lambda_{j} x}}\right) .
\end{aligned}
$$

Now, let

$$
\delta_{1}=\left(\sum_{i=1}^{n} \frac{\lambda_{i}^{2} e^{-\lambda_{i} x}}{\left(1-e^{-\lambda_{i} x}\right)^{2}} \sum_{j=1, j \neq i}^{n} \frac{\lambda_{j} e^{-\lambda_{j} x}}{1-e^{-\lambda_{j} x}}\right) \times\left(\frac{\bar{\lambda} e^{-\bar{\lambda} x}}{1-e^{-\bar{\lambda} x}}\right)\left(\sum_{i=1}^{n} \frac{\lambda_{i}}{1-e^{-\lambda_{i} x}}\right),
$$




$$
\begin{aligned}
\Delta_{1} & =\left(\sum_{i=1}^{n} \frac{\lambda_{i}}{1-e^{-\lambda_{i} x}} \sum_{j=1, j \neq i}^{n} \frac{\lambda_{j}^{2} e^{-\lambda_{j} x}}{\left(1-e^{-\lambda_{j} x}\right)^{2}}\right) \times\left(\frac{\bar{\lambda} e^{-\bar{\lambda} x}}{1-e^{-\bar{\lambda} x}}\right)\left(\sum_{i=1}^{n} \frac{\lambda_{i}}{1-e^{-\lambda_{i} x}}\right), \\
\delta_{2} & =\left(\frac{\bar{\lambda} e^{-\bar{\lambda} x}}{1-e^{-\bar{\lambda} x}}\right)\left(\sum_{i=1}^{n} \frac{\lambda_{i}^{2} e^{-\lambda_{i} x}}{\left(1-e^{-\lambda_{i} x}\right)^{2}}\right) \times\left(\sum_{i=1}^{n} \frac{\lambda_{i}}{1-e^{-\lambda_{i} x}} \sum_{j=1, j \neq i}^{n} \frac{\lambda_{j} e^{-\lambda_{j} x}}{1-e^{-\lambda_{j} x}}\right), \\
\Delta_{2} & =\left(\frac{\bar{\lambda}^{2} e^{-\bar{\lambda} x}}{\left(1-e^{-\bar{\lambda} x}\right)^{2}}\right)\left(\sum_{i=1}^{n} \frac{\lambda_{i}}{1-e^{-\lambda_{i} x}}\right) \times\left(\sum_{i=1}^{n} \frac{\lambda_{i}}{1-e^{-\lambda_{i} x}} \sum_{j=1, j \neq i}^{n} \frac{\lambda_{j} e^{-\lambda_{j} x}}{1-e^{-\lambda_{j} x}}\right),
\end{aligned}
$$

and let

$$
\lambda_{i}^{*}=\lambda_{i} x,
$$

then, (3.3.8) can be written as

$$
\delta_{1}+\Delta_{1} \leq \Delta_{2}+\delta_{2}
$$

First, we will prove

$$
\delta_{1} \leq \delta_{2}
$$

By Lemma 3.3.5,

$$
x^{5} \delta_{2} \geq \frac{n-1}{n}\left(\sum_{i=1}^{n} \frac{\lambda_{i}^{*}}{1-e^{-\lambda_{i}^{*}}}\right)\left(\sum_{i=1}^{n} \frac{\lambda_{i}^{*} e^{-\lambda_{i}^{*}}}{1-e^{-\lambda_{i}^{*}}}\right)\left(\frac{\bar{\lambda}^{*} e^{-\bar{\lambda}^{*} x}}{1-e^{-\bar{\lambda}^{*} x}}\right)\left(\sum_{i=1}^{n} \frac{\lambda_{i}^{*} e^{-\lambda_{i}^{*} x}}{\left(1-e^{-\lambda_{i}^{*} x}\right)^{2}}\right) .
$$

Hence, to $\delta_{1} \leq \delta_{2}$, it is enough to prove that,

$\sum_{i=1}^{n} \frac{\lambda_{i}^{* 2} e^{-\lambda_{i}^{*}}}{\left(1-e^{-\lambda_{i}^{*}}\right)^{2}} \sum_{j=1, j \neq i}^{n} \frac{\lambda_{j} e^{-\lambda_{j}^{*}}}{1-e^{-\lambda_{j}^{*}}} \leq \frac{n-1}{n}\left(\sum_{i=1}^{n} \frac{\lambda_{i}^{*} e^{-\lambda_{i}^{*}}}{1-e^{-\lambda_{i}^{*}}}\right)\left(\sum_{i=1}^{n} \frac{\lambda_{i}^{* 2} e^{-\lambda_{i}^{*}}}{\left(1-e^{\left.-\lambda_{i}^{*}\right)^{2}}\right.}\right)$.

That is,

$$
\frac{1}{n} \sum_{i=1}^{n} \frac{\lambda_{i}^{*} e^{-\lambda_{i}^{*}}}{1-e^{-\lambda_{i}^{*}}} \sum_{i=1}^{n} \frac{\lambda_{i}^{* 2} e^{-\lambda_{i}^{*}}}{\left(1-e^{-\lambda_{i}^{*}}\right)^{2}} \leq \sum_{i=1}^{n} \frac{\lambda_{i}^{* 3} e^{-2 \lambda_{i}^{*}}}{\left(1-e^{-\lambda_{i}^{*}}\right)^{3}}
$$


Observing that

$$
\frac{x e^{-x}}{1-e^{-x}} \text { and } \frac{x^{2} e^{-x}}{\left(1-e^{-x}\right)^{2}}
$$

are both decreasing, the inequality follows from Chebyshev's sum inequality.

Next, we will prove,

$$
\Delta_{1} \leq \Delta_{2}
$$

i.e.,

$$
\begin{aligned}
& \sum_{i=1}^{n} \frac{\lambda_{i}^{*}}{1-e^{-\lambda_{i}^{*}}} \sum_{j=1, j \neq i}^{n} \frac{\lambda_{j}^{* 2} e^{-\lambda_{j}^{*}}}{\left(1-e^{\left.-\lambda_{j}^{*}\right)^{2}}\right.} \\
\leq & \left(\frac{\bar{\lambda}^{*}}{1-e^{-\bar{\lambda}^{*}}}\right) \sum_{i=1}^{n} \frac{\lambda_{i}^{*}}{1-e^{-\lambda_{i}^{*}}} \sum_{j=1, j \neq i}^{n} \frac{\lambda_{j}^{*} e^{-\lambda_{j}^{*}}}{1-e^{-\lambda_{j}^{*}}} .
\end{aligned}
$$

That is,

$$
\frac{\sum_{i=1}^{n} \frac{\lambda_{i}^{*}}{1-e^{-\lambda_{i}^{*}}} \sum_{j=1, j \neq i}^{n} \frac{\lambda_{j}^{* 2} e^{-\lambda_{j}^{*}}}{\left(1-e^{-\lambda_{j}^{*}}\right)^{2}}}{\sum_{i=1}^{n} \frac{\lambda_{i}^{*}}{1-e^{-\lambda_{i}^{*}}} \sum_{j=1, j \neq i}^{n} \frac{\lambda_{j}^{*} e^{-\lambda_{j}^{*}}}{1-e^{-\lambda_{j}^{*}}}} \leq \frac{\bar{\lambda}^{*}}{1-e^{-\bar{\lambda}^{*}}} .
$$

Using Lemma 3.3.7, it is enough to prove,

$$
\frac{\sum_{j=1}^{n} \frac{\lambda_{j}^{* 2} e^{-\lambda_{j}^{*}}}{\left(1-e^{-\lambda_{j}^{*}}\right)^{2}}}{\sum_{j=1}^{n} \frac{\lambda_{j}^{*} e^{-\lambda_{j}^{*}}}{1-e^{-\lambda_{j}^{*}}}} \leq \frac{\bar{\lambda}^{*}}{1-e^{-\lambda^{*}}} .
$$

Using Lemma 3.3.6,

$$
\frac{\bar{\lambda}^{*}}{1-e^{-\lambda^{*}}} \geq \frac{n}{\sum_{i=1}^{n} \frac{1-e^{-\lambda_{i}^{*}}}{\lambda_{i}^{*}}}
$$


hence, it is sufficient to show,

$$
\frac{\sum_{j=1}^{n} \frac{\lambda_{j}^{* 2} e^{-\lambda_{j}^{*}}}{\left(1-e^{-\lambda_{j}^{*}}\right)^{2}}}{\sum_{j=1}^{n} \frac{\lambda_{j}^{*} e^{-\lambda_{j}^{*}}}{1-e^{-\lambda_{j}^{*}}}} \leq \frac{n}{\sum_{i=1}^{n} \frac{1-e^{-\lambda_{i}^{*}}}{\lambda_{i}^{*}}},
$$

i.e.,

$$
\sum_{i=1}^{n} \frac{1-e^{-\lambda_{i}^{*}}}{\lambda_{i}^{*}} \sum_{j=1}^{n} \frac{\lambda_{j}^{* 2} e^{-\lambda_{j}^{*}}}{\left(1-e^{-\lambda_{j}^{*}}\right)^{2}} \leq n \sum_{j=1}^{n} \frac{\lambda_{j}^{*} e^{-\lambda_{j}^{*}}}{1-e^{-\lambda_{j}^{*}}} .
$$

Since as mentioned in Lemma 2.2.1, the functions

$$
\frac{1-e^{-x}}{x} \text { and } \frac{x^{2} e^{-x}}{\left(1-e^{-x}\right)^{2}}
$$

are both decreasing, the above inequality follows immediately from Chebyshev's sum inequality.

Hence, the required result follows.

Now, we are ready to prove the result for the likelihood ratio order.

Theorem 3.3.9 Let $X_{1}, \ldots, X_{n}$ be independent exponential random variables with $X_{i}$ having hazard rate $\lambda_{i}, i=1, \ldots, n$. Let $Y_{1}, \ldots, Y_{n}$ be a random sample of size $n$ from an exponential distribution with common hazard rate $\bar{\lambda}$, then,

$$
X_{n: n}-X_{1: n} \geq_{l r} Y_{n: n}-Y_{1: n}
$$

Proof: Since the density function of $R_{X}=X_{n: n}-X_{1: n}$ can written as,

$$
f_{R_{X}}(x)=\tilde{r}_{R_{X}}(x) F_{R_{X}}
$$


thus,

$$
\frac{f_{R_{X}}(x)}{f_{R_{Y}}(x)}=\frac{\tilde{r}_{R_{X}}(x)}{\tilde{r}_{R_{Y}}(x)} \frac{F_{R_{X}(x)}}{F_{R_{Y}}(x)} .
$$

From Theorem 3.3.7,

$$
\frac{F_{R_{X}(x)}}{F_{R_{Y}}(x)} \text { is increasing in } x \geq 0 \text {. }
$$

Combining it with Theorem 3.3.8, the result follows.

Remark: Observing that $X_{1: n}$ and $Y_{1: n}$ have the same logconcave distribution, it from Shanthikumar and Yao [61] that the above result implies that

$$
X_{n: n} \geq_{l r} Y_{n: n},
$$

a result already proved in Lemma 2.2.2.

One natural question is whether our result could be improved to the case of majorization? For instance, is it true that if $X_{1}, \cdots, X_{n}$ are independent exponentials with hazard rates $\theta_{1}, \cdots, \theta_{n}$ and $Y_{1}, \cdots, Y_{n}$ are independent exponentials with hazard rates $\lambda_{1}, \cdots, \lambda_{n}$, then

$$
\left(\theta_{1}, \cdots, \theta_{n}\right) \succeq_{\mathrm{m}}\left(\lambda_{1}, \cdots, \lambda_{n}\right)
$$

implies that $\tilde{r}_{R_{X}}(x) / \tilde{r}_{R_{Y}}(x)$ is increasing in $x \in(0, \infty)$ ? The following example gives a negative answer.

Example 3.3.10 Let $\left(X_{1}, X_{2}, X_{3}\right)$ be independent exponential random vector with hazard rate vector $\left(\theta_{1}, \theta_{2}, \theta_{3}\right)=(0.1,1,9)$, and $\left(Y_{1}, Y_{2}, Y_{3}\right)$ be independent exponential random vector with hazard rate vector $\left(\lambda_{1}, \lambda_{2}, \lambda_{3}\right)=(0.1,4,6)$. It is easily 
seen that

$$
\left(\theta_{1}, \theta_{2}, \theta_{3}\right) \succeq_{\mathrm{m}}\left(\lambda_{1}, \lambda_{2}, \lambda_{3}\right)
$$

However,

$$
\frac{\tilde{r}_{R_{X}}(1)}{\tilde{r}_{R_{Y}}(1)}=1.49457>\frac{\tilde{r}_{R_{X}}(2)}{\tilde{r}_{R_{Y}}(2)}=1.32433 .
$$

\subsubsection{Dispersive order}

The dispersive order between the ranges will be proved in the following Theorem.

Theorem 3.3.11 Let $X_{1}, \ldots, X_{n}$ be independent exponential random variables with $X_{i}$ having hazard rate $\lambda_{i}, i=1, \ldots, n$. Let $Y_{1}, \ldots, Y_{n}$ be a random sample of size $n$ from an exponential distribution with common hazard rate $\bar{\lambda}$, then,

$$
X_{n: n}-X_{1: n} \geq_{d i s p} Y_{n: n}-Y_{1: n} .
$$

Proof: Note that, the distribution function of $R_{Y}$ is, for $x \geq 0$,

$$
F_{R_{Y}}(x)=\left(1-e^{-\bar{\lambda} x}\right)^{n-1}
$$

Since, from Theorem 3.3.2,

$$
R_{X} \geq_{s t} R_{Y}
$$

that is, for $x \geq 0$,

$$
F_{R_{Y}}(x) \geq F_{R_{X}}(x) .
$$

Hence, for any $c \leq 0, x \geq 0$,

$$
F_{R_{Y}}(x-c) \geq F_{R_{X}}(x) .
$$


Now, we consider the case of $c>0$.

Assume there exists at least two points $x_{1}$ and $x_{2}$, such that, for $x_{2}>x_{1}>0$,

$$
\begin{aligned}
& F_{R_{Y}}\left(x_{1}-c\right)=F_{R_{X}}\left(x_{1}\right), \\
& F_{R_{Y}}\left(x_{2}-c\right)=F_{R_{X}}\left(x_{2}\right) .
\end{aligned}
$$

We only need to consider when $x_{1}>c$. The other case is trivial. Note that,

$$
F_{R_{Y}}\left(x_{1}-c\right)=F_{R_{X}}\left(x_{1}\right)
$$

i.e.,

$$
\left(1-e^{-\bar{\lambda}\left(x_{1}-c\right)}\right)^{n-1}=F_{R_{X}}\left(x_{1}\right)
$$

could be rewritten as

$$
c=x_{1}+\frac{1}{\bar{\lambda}} \ln \left(1-F_{R_{X}}^{1 /(n-1)}\left(x_{1}\right)\right) .
$$

Similarly,

$$
c=x_{2}+\frac{1}{\bar{\lambda}} \ln \left(1-F_{R_{X}}^{1 /(n-1)}\left(x_{2}\right)\right) .
$$

Hence, it follows that,

$$
x_{2}-x_{1}=\frac{1}{\bar{\lambda}} \ln \left(\frac{1-F_{R_{X}}^{1 /(n-1)}\left(x_{1}\right)}{1-F_{R_{X}}^{1 /(n-1)}\left(x_{2}\right)}\right),
$$

which is equivalent to

$$
e^{\bar{\lambda}\left(x_{2}-x_{1}\right)}=\frac{1-F_{R_{X}}^{1 /(n-1)}\left(x_{1}\right)}{1-F_{R_{X}}^{1 /(n-1)}\left(x_{2}\right)},
$$


i.e.,

$$
\frac{1-F_{R_{X}}^{1 /(n-1)}\left(x_{1}\right)}{e^{-\bar{\lambda} x_{1}}}=\frac{1-F_{R_{X}}^{1 /(n-1)}\left(x_{2}\right)}{e^{-\bar{\lambda} x_{2}}} .
$$

Now, consider, for $x \geq 0$,

$$
1-F_{R_{X}}^{1 /(n-1)}(x)
$$

as a survival function. Hence, the density function is

$$
\frac{1}{n-1} F_{R_{X}}^{1 /(n-1)}(x) \tilde{r}_{R_{X}}(x)
$$

Similarly, considering, for $x \geq 0, e^{-\bar{\lambda} x}$ as a survival function, the density is,

$$
\bar{\lambda} e^{-\bar{\lambda} x}
$$

Note that

$$
\begin{aligned}
& \frac{\frac{1}{n-1} F_{R_{X}}^{1 /(n-1)}(x) \tilde{r}_{R_{X}}(x)}{\bar{\lambda} e^{-\bar{\lambda} x}} \\
= & \frac{1}{n-1} \frac{F_{R_{X}}^{1 /(n-1)}(x)}{1-e^{-\bar{\lambda} x}} \times \frac{\tilde{r}_{R_{X}}(x)}{\frac{\bar{\lambda} e^{-\bar{\lambda} x}}{1-e^{-\bar{\lambda} x}}} \\
= & \left(\frac{F_{R_{X}}(x)}{F_{R_{Y}}(x)}\right)^{1 /(n-1)} \frac{\tilde{r}_{R_{X}}(x)}{\tilde{r}_{R_{Y}}(x)}
\end{aligned}
$$

Since, for $x \geq 0$,

$$
\frac{\tilde{r}_{R_{X}}(x)}{\tilde{r}_{R_{Y}}(x)}
$$

is strictly increasing, except for $\lambda_{1}=\lambda_{2}=\cdots=\lambda_{n}$ as proved in Theorem 3.3.8, and since,

$$
\frac{F_{R_{X}(x)}}{F_{R_{Y}}(x)}
$$


is increasing in $x \geq 0$, it follows that

$$
\frac{\frac{1}{n-1} F_{R_{X}}^{1 /(n-1)}(x) \tilde{r}_{R_{X}}(x)}{\bar{\lambda} e^{-\bar{\lambda} x}}
$$

is strictly increasing in $x \geq 0$. Thus,

$$
\frac{1-F_{R_{X}}^{1 /(n-1)}(x)}{e^{-\bar{\lambda} x}}
$$

is strictly increasing in $x \geq 0$, as the likelihood ratio order implies the hazard rate order. However, this contradicts to Eq. (3.3.9). Thus, we only have at most one crossing. If the crossing occurs, the sign should be from - to + . Hence, the desired result follows.

Dr. Ali Dolati suggested the following proof in our personal communication.

Proof. Put $\ell=1 /(n-1)>0$ and let $X_{\ell}$ and $Y_{\ell}$ be distributed as $F_{R_{X}}^{\ell}$ and $F_{R_{Y}}^{\ell}$, respectively. The ratio of their densities may then be expressed as a product of positive increasing functions, viz.

$$
\frac{\ell F_{R_{X}}^{\ell-1}(t) f_{R_{X}}(t)}{\ell F_{R_{Y}}^{\ell-1}(t) f_{R_{Y}}(t)}=\left(\frac{F_{R_{X}}(t)}{F_{R_{Y}}(t)}\right)^{\ell} \times \frac{\tilde{r}_{R_{X}}(t)}{\tilde{r}_{R_{Y}}(t)} .
$$

Accordingly, one has $F_{R_{Y}}^{\ell} \leq_{l r} F_{R_{X}}^{\ell}$ and hence $Y_{\ell}$ is smaller than $X_{\ell}$ in the hazard rate ordering. Thus the mapping

$$
t \mapsto H_{\ell}(t)=\frac{1-F_{R_{X}}^{\ell}(t)}{1-F_{R_{Y}}^{\ell}(t)}=\frac{1-F_{R_{X}}^{\ell}(t)}{e^{-\lambda t}}
$$

is increasing on $\mathbb{R}_{+}$. Clearly, $\mathrm{d} H_{\ell}(t) / \mathrm{d} t \geq 0$ if and only if

$$
f_{R_{X}}(t) \leq \lambda(n-1) F_{R_{X}}(t)\left\{F_{R_{X}}^{-\ell}(t)-1\right\}
$$


for all $t \in \mathbb{R}_{+}$. As the right-hand side equals $f_{R_{Y}} \circ{F_{R_{Y}}}^{-1} \circ F_{R_{X}}(t)$, it follows that $f_{R_{X}} \circ F_{R_{X}}{ }^{-1}(u) \leq f_{R_{Y}} \circ F_{R_{Y}}{ }^{-1}(u)$ for all $u \in(0,1)$, whence $R_{Y} \leq_{\text {disp }} R_{X}$ by Equation (3.B.11) of Shaked and Shanthikumar [60].

Remark 3. In the case $n=2$, Theorem 3.3.11 also follows from Theorem 3.7 in Kochar and Korwar [34], which states that under the conditions of Theorem 3.3.11, the normalized spacings of the homogeneous sample are less dispersed than those of the heterogeneous sample.

The following result is an immediately consequence of Theorem 3.3.11.

Corollary 3.3.12 Let $X_{1}, \ldots, X_{n}$ be independent exponential random variables with $X_{i}$ having hazard rate $\lambda_{i}, i=1, \ldots, n$. Let $Y_{1}, \ldots, Y_{n}$ be a random sample of size $n$ from an exponential distribution with common hazard rate $\bar{\lambda}$, then,

$$
\operatorname{Var}\left\{X_{n: n}-X_{1: n}\right\} \geq \operatorname{Var}\left\{Y_{n: n}-Y_{1: n}\right\}
$$

\subsection{Application}

An $n$-component system that fails if and only if at least $k$ of the $n$ components fail is called a $k$-out-of- $n$ : F system. The lifetime of such a system could be represented as $X_{k: n}$. The $k$-out-of- $n$ system structure is a very popular type of redundancy in fault tolerant systems with wide applications in industrial and military systems. For two different systems say a $k$-out-of- $n$ : F system and a $k+1$-out-of- $n$ : F system, the engineer may be interested in the additional lifetime 
$X_{k+1: n}-X_{k: n}$ for the system design and the cost purpose. Due to the complicated distribution form, one may provide a sharp bound on the survival function of this based on Theorem 3.2.2.

For example, a plane has four engines, and a minimum of three engines are required for the plane work. Hence, this plane is a 2-out-of-4: F system. If the engineer is planing to improve the system to the 3-out-of- 4 : F system, he/she has to consider the cost and the reliability of improvement simultaneously. Hence, it is important the estimate the survival probability and the mean additional lifetime $X_{3: 4}-X_{2: 4}$. Theorem 3.2.2 provides a lower bound for the survival function of the additional lifetime. Let $\left(\lambda_{1}, \lambda_{2}, \lambda_{3}, \lambda_{4}\right)=(1,2,2.5,3)$, then,

$$
P\left(X_{3: 4}-X_{2: 4}>x\right) \geq e^{-0.955 x}
$$

Using Theorem 3.2.3, the following sharp lower bound could be established,

$$
\mathrm{E}\left(X_{3: 4}-X_{2: 4}\right) \geq 1.0879
$$

In the following table, we list the lower bounds on $\lambda$ 's for various partial orders to hold in Theorems 3.2.1-3.2.3.

The values above are comparable to the arithmetic mean 2.125 , geometric mean 1.96799 and harmonic mean 1.791045 of $\lambda_{i}$ 's. It is interesting to note that all of the values are less than the arithmetic mean, which coincides with the condition in Theorem 3.2.3. 


\begin{tabular}{|c|c|c|c|}
\hline$\lambda$ & $\mathrm{lr} / \mathrm{rh}$ & $\mathrm{hr} / \mathrm{disp} / \mathrm{st}$ & $\mathrm{mrl} / \mathrm{RS} / \mathrm{E}$ \\
\hline$D_{2: 4}$ & 2.060897 & 2.039216 & 2.019271 \\
$D_{3: 4}$ & 1.988454 & 1.909626 & 1.838388 \\
$D_{4: 4}$ & 2.009722 & 1.674501 & 1.382051 \\
\hline
\end{tabular}

Table 3.1: Parameters for different orders

\subsection{Concluding remarks}

In this Chapter, equivalent characterizations of stochastic comparisons of spacings between heterogeneous samples and homogeneous samples are established. Sufficient conditions for comparing the ranges are also provided. There are still many directions in which this research might be continued. For example, let $X_{1}, \ldots, X_{n}$ be independent exponential random variables with $X_{i}$ having hazard rate $\lambda_{i}, i=1, \ldots, n$. Let $Y_{1}, \ldots, Y_{n}$ be a random sample of size $n$ from an exponential distribution with common hazard rate $\bar{\lambda}$. Then, for $k=3, \cdots, n-1$, whether

$$
X_{k: n}-X_{1: n} \geq_{l r(h r)} Y_{k: n}-Y_{1: n}
$$

is true?

Observing Table 3.1, one can make the following conjecture, for $k \geq 2$,

$$
D_{2: n} \geq_{h r(m r l)} H_{2: n} \Rightarrow D_{k: n} \geq_{h r(m r l)} H_{k: n} .
$$

The material of this Chapter is mainly based on Kochar and Xu [39] and Genest, 
Kochar and $X u$ [23]. 


\section{Chapter 4}

\section{Dependence between extreme order statistics}

\subsection{Introduction}

It is interesting to know the dependence properties of order statistics. Boland et al. [12] showed that if $X_{1}, \ldots, X_{n}$ are independent random variables with differentiable densities and proportional hazard functions on an interval, then $X_{i: n}$ is SI in $X_{1: n}$. However, in general, $X_{i: n}$ is not SI in $X_{1: n}$, as illustrated by a counterexample there. The other interesting topic is to study the relative degree of dependence that may exist between two arbitrary pairs of order statistics, say $\left(X_{i: n}, X_{j: n}\right)$ and $\left(X_{i^{\prime}: n}, X_{j^{\prime}: n}\right)$. Intuitively, when order statistics $X_{i: n}$ and $X_{j: n}$ draw apart, they should tend to be less dependent. One nature way to compare two pairs of order statistics is the traditional notion of covariance (cf. Kim and David [32]). However, as pointed out in Avérous et al. [1], it is not an appropriate measure of dependence when the pairs being compared do not have the same marginal distributions. They proposed a copula-based dependence order, called more SI order, which enables to compare two pairs of random variables with different margins. 
This order has the advantage that it implies the well known weaker dependence orders like more PQD (or more concordance) order. As mentioned in Avérous et al [1], more PQD order implies

$$
\kappa\left(X_{1}, Y_{1}\right) \leq \kappa\left(X_{2}, Y_{2}\right)
$$

where $\kappa(X, Y)$ represents Spearman's rho, Kendall's tau, Gini's coefficient, or any other copula-based measure of concordance satisfying the axioms of Scarsini [57].

Avérous et al. [1] proved there for $i<j$, the dependence of the $j$ th order statistic on the $i$ th order statistic decreases as $i$ and $j$ draw apart in the sense of more SI dependence order when $X_{i}$ are i.i.d. random variables. Recently, Kochar and Xu [40] proved that this result also holds in the sense of more reversed hazard rate (RHR) dependence order, which is stronger than the more SI dependence order. However, in practice, systems are often made up of components whose lifetimes $X_{1}, X_{2}, \cdots, X_{n}$ are not identically distributed. One question arises naturally: For two systems, one composed of i.i.d. components, and the other with independent non-identical components, which system has more dependence between a pair of order statistics? Sathe [56] first proved that

$$
\operatorname{corr}\left(Y_{1: n}, Y_{n: n}\right) \leq \operatorname{corr}\left(X_{1: n}, X_{n: n}\right)
$$

where $X_{1}, \cdots, X_{n}$ are mutually independent exponentials with distinct hazard rates $\lambda_{1}, \cdots, \lambda_{n}$, but $Y_{1}, \cdots, Y_{n}$ is a random sample from the exponential dis- 
tribution with hazard rate $\bar{\lambda}=\left(\lambda_{1}+\cdots+\lambda_{n}\right) / n$. However, it only compares the relative degree of linear association within the two pairs under special distributions. Recently, Dolati et al. [18] used two copula-based dependence orders called more right-tail increasing (RTI) and more left-tail decreasing (LTD), to investigate the relative dependence between the extreme order statistics when one sample consists of independent but non-identically distributed observations with proportional hazard rates, but the other sample with i.i.d. observations. It is shown that

$$
\left(X_{n: n} \mid X_{1: n}\right) \prec_{\mathrm{RTI}}\left(Y_{n: n} \mid Y_{1: n}\right)
$$

where $X_{1}, \cdots, X_{n}$ are mutually independent random variables with distinct hazard rates $\lambda_{i}(t)=r_{i} \lambda^{*}(t)$, but $Y_{1}, \cdots, Y_{n}$ is a random sample from the distribution with common hazard rate $\lambda(t)$. Observing more RTI order implies more PQD order, it follows that,

$$
\kappa\left(X_{n: n}, X_{1: n}\right) \leq \kappa\left(Y_{1: n}, Y_{n: n}\right)
$$

Since more RTI order is weaker than more SI order, they also propose one open problem there: Whether (4.1.1) could be generalized to more SI order? In the following section, we will answer this question, by showing,

$$
\left(X_{n: n} \mid X_{1: n}\right) \prec_{\mathrm{SI}}\left(Y_{n: n} \mid Y_{1: n}\right) .
$$




\subsection{SI dependence order}

Theorem 4.2.1 Let $X_{1}, \ldots, X_{n}$ be independent continuous random variables with $X_{i}$ having survival function $\bar{F}^{\lambda_{i}}, i=1, \ldots, n$. Let $Y_{1}, \ldots, Y_{n}$ be i.i.d. continuous random variables, then

$$
\left(X_{n: n} \mid X_{1: n}\right) \prec_{\mathrm{SI}}\left(Y_{n: n} \mid Y_{1: n}\right)
$$

Proof: Without loss of generality, we assume that $X_{i}$ 's are exponentially distributed with parameters $\lambda_{1}, \lambda_{2}, \ldots, \lambda_{n}$, since the more SI dependence order is copula based. Observing that $\left(Y_{n: n} \mid Y_{1: n}\right)$ has the same copula structure with $\left(U_{n: n} \mid U_{1: n}\right)$ (see Avérous, et al. [1]), where $U_{n: n}$ and $U_{1: n}$ are extreme order statistics from uniform distribution. Hence, we can also assume $Y_{i}, i=1, \cdots, n$ have common population survival distribution $\bar{G}=\bar{F}^{\bar{\lambda}}$, where $\bar{\lambda}=\sum_{i=1}^{n} \lambda_{i} / n$.

Since $X_{n: n}-X_{1: n}$ is independent of $X_{1: n}$ (cf. Kochar and Korwar [34]), it follows that, for $u>s \geq 0$,

$$
\begin{aligned}
H_{1[s]}(u) & =P\left[X_{n: n} \leq u \mid X_{1: n}=s\right] \\
& =P\left[X_{n: n}-X_{1: n} \leq u-s \mid X_{1: n=s}\right] \\
& =F_{R_{X}}(u-s)
\end{aligned}
$$

where $F_{R_{X}}$ denotes the distribution function of $R_{X}$. Let $\xi_{p}$ denote the $p$ th quantile of the common distribution of $X_{1: n}$ and $Y_{1: n}$. Therefore,

$$
H_{1\left[\xi_{p}\right]}^{-1}(u)=F_{R_{X}}^{-1}(u)+\xi_{p}
$$


and, for $0 \leq p<q \leq 1$,

$$
H_{\left[1 \xi_{q}\right]} \circ H_{\left[1 \xi_{p}\right]}^{-1}(u)=F_{R_{X}}\left[F_{R_{X}}^{-1}(u)-\left(\xi_{q}-\xi_{p}\right)\right] .
$$

Similarly,

$$
H_{\left[2 \xi_{q}\right]} \circ H_{\left[2 \xi_{p}\right]}^{-1}(u)=G_{R_{Y}}\left[G_{R_{Y}}^{-1}(u)-\left(\xi_{q}-\xi_{p}\right)\right],
$$

where $G_{R_{Y}}$ denotes the distribution function of $R_{Y}$. According to the definition, we need to prove

$$
G_{R_{Y}}\left[G_{R_{Y}}^{-1}(u)-\left(\xi_{q}-\xi_{p}\right)\right] \leq F_{R_{X}}\left[F_{R_{X}}^{-1}(u)-\left(\xi_{q}-\xi_{p}\right)\right],
$$

i.e.,

$$
Y_{n: n}-Y_{1: n} \leq_{d i s p} X_{n: n}-X_{1: n}
$$

which is true as proved in the Theorem 3.3.11.

The main result in this Chapter is based on Genest, Kochar and Xu [23]. 


\section{Chapter 5}

\section{Stochastic comparisons of convolutions of independent random variables}

\subsection{Introduction}

The exponential distribution is one of most popular distributions in probability and statistics. In reliability theory, it is well-known for its "non-aging" property and also has many interesting applications in operation research. It has also been widely used in queuing theory, survival analysis and physics. Please refer to Barlow and Proschan [8] and Balakrishnan and Basu [5] for more details. The convolution of exponential random variables has attracted considerable attention in the literature due to its typical applications in many areas. For example, in reliability theory, it arises in the study of redundant standby systems with exponential components (cf. Bon and Păltănea [13]); in queuing theory, it is used to model the total service time of an agent in a system; in insurance, it is used to model total claims on a number of policies in the individual risk model (cf. Kaas et al. [25]).

Let $X_{1}, \ldots, X_{n}$ be independent exponential random variables with $X_{i}$ having hazard rate $\lambda_{i}, i=1, \ldots, n$, and $Y_{1}, \ldots, Y_{n}$ be another set of independent expo- 
nential random variables with with $Y_{i}$ with hazard rate $\lambda_{i}^{\prime}, i=1, \ldots, n$. Boland et al. [11] showed that under the condition of the majorization order,

$$
\left(\lambda_{1}, \cdots, \lambda_{n}\right) \succeq_{\mathrm{m}}\left(\lambda_{1}^{\prime}, \cdots, \lambda_{n}^{\prime}\right) \Longrightarrow \sum_{i=1}^{n} X_{i} \geq_{l r} \sum_{i=1}^{n} Y_{i} .
$$

Under the same condition, Kochar and $M a$ [35] proved that

$$
\left(\lambda_{1}, \cdots, \lambda_{n}\right) \succeq_{\mathrm{m}}\left(\lambda_{1}^{\prime}, \cdots, \lambda_{n}^{\prime}\right) \Longrightarrow \sum_{i=1}^{n} X_{i} \geq_{\text {disp }} \sum_{i=1}^{n} Y_{i}
$$

This topic has been extensively investigated by Bon and Păltănea [13]. They pointed out that, under the $p$ order, which is a weaker order than the majorization order,

$$
\left(\lambda_{1}, \cdots, \lambda_{n}\right) \succeq\left(\lambda_{1}^{\prime}, \cdots, \lambda_{n}^{\prime}\right) \Longrightarrow \sum_{i=1}^{n} X_{i} \geq_{h r} \sum_{i=1}^{n} Y_{i} .
$$

This result has been strengthened by Khaledi and Kochar [30] as

$$
\left(\lambda_{1}, \cdots, \lambda_{n}\right) \succeq^{p}\left(\lambda_{1}^{\prime}, \cdots, \lambda_{n}^{\prime}\right) \Longrightarrow \sum_{i=1}^{n} X_{i} \geq_{\text {disp }} \sum_{i=1}^{n} Y_{i}
$$

More recently, Zhao and Balakrishnan [68] proved that, under the condition of reciprocal order,

$$
\left(\lambda_{1}, \cdots, \lambda_{n}\right) \stackrel{\mathrm{rm}}{\succeq}\left(\lambda_{1}^{\prime}, \cdots, \lambda_{n}^{\prime}\right) \Longrightarrow \sum_{i=1}^{n} X_{i} \geq_{m r l} \sum_{i=1}^{n} Y_{i}
$$

Observing (5.1.2), one natural question is to find sufficient condition on the parameters which will imply right spread order between two such convolutions of independent exponential random variables. This has been an open problem for a 
long time because it is very complicated to check it from the definition of right spread order. In this section, we will solve this problem by showing that,

$$
\left(\lambda_{1}, \cdots, \lambda_{n}\right) \succeq\left(\lambda_{1}^{\prime}, \cdots, \lambda_{n}^{\prime}\right) \Longrightarrow \sum_{i=1}^{n} X_{i} \geq_{R S} \sum_{i=1}^{n} Y_{i}
$$

This new result not only complements the existing results on variability between convolutions in the literature, it also gives a new insight into the distribution theory of convolutions. Using (5.1.4), it is further proved that,

$$
\left(\frac{1}{\lambda_{1}}, \cdots, \frac{1}{\lambda_{n}}\right) \succeq_{\mathrm{m}}\left(\frac{1}{\lambda_{1}^{\prime}}, \cdots, \frac{1}{\lambda_{n}^{\prime}}\right) \Longrightarrow \sum_{i=1}^{n} X_{i} \geq_{\mathrm{NBUE}} \sum_{i=1}^{n} Y_{i},
$$

where " $\geq_{\text {NBUE" }}$ means NBUE order. NBUE order is a partial order to compare the aging of two distributions. It can be used to compare relative skewness in two distributions (cf. Kochar and Wiens [38] and Marshall and Olkin [48]). It will be shown that the convolution of heterogeneous exponential random variables is more skewed than that of homogeneous exponential random variables. Since NBUE order implies Lorenz ordering, we prove

$$
\left(\frac{1}{\lambda_{1}}, \cdots, \frac{1}{\lambda_{n}}\right) \succeq_{\mathrm{m}}\left(\frac{1}{\lambda_{1}^{\prime}}, \cdots, \frac{1}{\lambda_{n}^{\prime}}\right) \Longrightarrow \sum_{i=1}^{n} X_{i} \geq_{\text {Lorenz }} \sum_{i=1}^{n} Y_{i},
$$

where " $\geq_{\text {Lorenz }}$ " is the well-known Lorenz order, which may be of independent interest in economics.

\subsection{Right spread order}

Saunders and Moran [51] established an equivalent characterization of dispersive 
order in one parameter family. The following lemma gives an equivalent characterization of right spread order in one parameter family.

Lemma 5.2.1 Let $\left\{F_{a} \mid a \in R\right\}$ be a class of distribution functions, such that $F_{a}$ is supported on some interval $\left(x_{-}^{(a)}, x_{+}^{(a)}\right) \subseteq(0, \infty)$, where $x_{-}^{(a)}$ and $x_{+}^{(a)}$ mean the left and right end points, respectively. Then,

$$
X_{a^{*}} \leq_{R S} X_{a}, \quad a \leq a^{*},
$$

if and only if

$$
\frac{W_{a}^{\prime}(x)}{\bar{F}_{a}(x)} \text { is decreasing in } x,
$$

where $W_{a}^{\prime}$ is the derivative of $W_{a}$ with respect to $a$.

Proof: Note that $X_{a^{*}} \leq_{R S} X_{a}$ for $a \leq a^{*}$, if and only if,

$$
W_{a}^{-1}(\beta)-W_{a^{*}}^{-1}(\beta) \leq W_{a}^{-1}(\alpha)-W_{a^{*}}^{-1}(\alpha), \quad \beta \geq \alpha
$$

i.e., for $\alpha \leq \beta$,

$$
W_{a}^{-1}(\alpha)-W_{a}^{-1}(\beta)
$$

is decreasing in $a$. This condition is equivalent to,

$$
W_{a}\left(W_{a}^{-1}(\alpha)+c\right), \quad \text { decreasing in } a \text { for } c \geq 0 \text {. }
$$

To see this, let $\alpha=W_{a}\left(W_{a}^{-1}(\beta)+c\right)$ as $W_{a}^{-1}(\cdot)$ is a decreasing function. If (5.3.4) holds, for any $\lambda \geq 0$,

$$
W_{a+\lambda}\left(W_{a+\lambda}^{-1}(\beta)+c\right) \leq W_{a}\left(W_{a}^{-1}(\beta)+c\right)=\alpha=W_{a+\lambda}\left(W_{a+\lambda}^{-1}(\alpha)\right),
$$


i.e., for $\alpha \leq \beta$,

$$
W_{a+\lambda}^{-1}(\alpha)-W_{a+\lambda}^{-1}(\beta) \leq c=W_{a}^{-1}(\alpha)-W_{a}^{-1}(\beta), \quad \lambda \geq 0 .
$$

So, (5.3.4) implies (5.2.1). Reversing the above argument leads (5.2.1) implies (5.3.4).

Next, by taking derivative with respect to $a,(5.3 .4)$ is equivalent to

$$
W_{a}^{\prime}\left(W_{a}^{-1}(\alpha)+c\right)-\bar{F}_{a}\left(W_{a}^{-1}(\alpha)+c\right) W_{a}^{\prime-1}(\alpha) \leq 0,
$$

where $W_{a}^{\prime}=\partial W_{a} / \partial a$ and $W_{a}^{\prime-1}(\alpha)=\partial W_{a}^{-1} / \partial a$.

Observe that,

$$
W_{a}\left(W_{a}^{-1}(\alpha)\right)=\alpha
$$

Taking derivative with respect to $a$ for both sides, it gives,

$$
W_{a}^{\prime}\left(W_{a}^{-1}(\alpha)\right)-\bar{F}_{a}\left(W_{a}^{-1}(\alpha)\right) W_{a}^{\prime-1}(\alpha)=0 .
$$

Use this equation for (5.3.5), it follows that, for $c \geq 0$,

$$
\frac{W_{a}^{\prime}\left(W_{a}^{-1}(\alpha)+c\right)}{\bar{F}_{a}\left(W_{a}^{-1}(\alpha)+c\right)} \leq \frac{W_{a}^{\prime}\left(W_{a}^{-1}(\alpha)\right)}{\bar{F}_{a}\left(W_{a}^{-1}(\alpha)\right)} .
$$

Hence, the required result follows immediately.

Now, let us discuss convolutions of independent exponential random variables when $n=2$. 
Theorem 5.2.2 Let $X_{\lambda_{1}}, X_{\lambda_{2}}, X_{\lambda_{1}^{\prime}}, X_{\lambda_{2}^{\prime}}$ be independent exponential random variables with parameters $\lambda_{1}, \lambda_{2}, \lambda_{1}^{\prime}, \lambda_{2}^{\prime}$, respectively. Then,

$$
\left(\lambda_{1}, \lambda_{2}\right) \stackrel{\mathrm{rm}}{\succeq}\left(\lambda_{1}^{\prime}, \lambda_{2}^{\prime}\right) \Longleftrightarrow X_{\lambda_{1}}+X_{\lambda_{2}} \geq_{R S} X_{\lambda_{1}^{\prime}}+X_{\lambda_{2}^{\prime}}
$$

Proof:“Sufficiency $\Longrightarrow$ ”. Without loss of generality, assume that $\lambda_{1} \leq \lambda_{2}$ and $\lambda_{1}^{\prime} \leq \lambda_{2}^{\prime}$. As

$$
\left(\lambda_{1}, \lambda_{2}\right) \stackrel{\mathrm{rm}}{\succeq}\left(\lambda_{1}^{\prime}, \lambda_{2}^{\prime}\right)
$$

it holds that

$$
\frac{1}{\lambda_{1}} \geq \frac{1}{\lambda_{1}^{\prime}}
$$

and

$$
\frac{1}{\lambda_{1}}+\frac{1}{\lambda_{2}} \geq \frac{1}{\lambda_{1}^{\prime}}+\frac{1}{\lambda_{2}^{\prime}} .
$$

Now, let us discuss several cases.

\section{(i) Case 1:}

$$
\frac{1}{\lambda_{2}} \geq \frac{1}{\lambda_{1}^{\prime}}+\frac{1}{\lambda_{2}^{\prime}}
$$

Since

$$
\frac{1}{\lambda_{1}} \geq \frac{1}{\lambda_{1}^{\prime}} \Longrightarrow X_{\lambda_{1}} \geq_{R S} X_{\lambda_{1}^{\prime}}
$$

it holds that

$$
X_{\lambda_{1}} \geq_{R S} X_{\lambda_{1}^{\prime}}, \quad X_{\lambda_{2}} \geq_{R S} X_{\lambda_{2}^{\prime}}
$$


From Theorem 3.C.7 of Shaked and Shanthikumar [60] (see also Hu, et al. [24]), it follows that,

$$
X_{\lambda_{1}}+X_{\lambda_{2}} \geq_{R S} X_{\lambda_{1}^{\prime}}+X_{\lambda_{2}^{\prime}} .
$$

\section{(ii) Case 2:}

$$
\frac{1}{\lambda_{2}}<\frac{1}{\lambda_{1}^{\prime}}+\frac{1}{\lambda_{2}^{\prime}},
$$

but

$$
\frac{1}{\lambda_{1}} \geq \frac{1}{\lambda_{1}^{\prime}}+\frac{1}{\lambda_{2}^{\prime}} .
$$

Denote

$$
\frac{1}{\lambda_{1}^{*}}=\frac{1}{\lambda_{1}^{\prime}}+\frac{1}{\lambda_{2}^{\prime}} .
$$

From Theorem 3. C. 8 of Shaked and Shanthikumar [60],

$$
X_{\lambda_{1}}+X_{\lambda_{2}} \geq_{R S} X_{\lambda_{1}},
$$

observing that,

$$
\frac{1}{\lambda_{1}} \geq \frac{1}{\lambda_{1}^{*}} \Longrightarrow X_{\lambda_{1}} \geq_{R S} X_{\lambda_{1}^{*}},
$$

it is sufficient to show

$$
X_{\lambda_{1}^{*}} \geq_{R S} X_{\lambda_{1}^{\prime}}+X_{\lambda_{2}^{\prime}} .
$$

Now, since

$$
\frac{1}{\lambda_{1}^{*}}=\frac{1}{\lambda_{1}^{\prime}}+\frac{1}{\lambda_{2}^{\prime}},
$$


i.e.,

$$
\mathrm{E}\left(X_{\lambda_{1}^{*}}\right)=\mathrm{E}\left(X_{\lambda_{1}^{\prime}}\right)+\mathrm{E}\left(X_{\lambda_{2}^{\prime}}\right)
$$

it holds that

$$
X_{\lambda_{1}^{*}} \geq_{R S} X_{\lambda_{1}^{\prime}}+X_{\lambda_{2}^{\prime}} \Longleftrightarrow X_{\lambda_{1}^{*}} \geq_{\mathrm{NBUE}} X_{\lambda_{1}^{\prime}}+X_{\lambda_{2}^{\prime}}
$$

Hence, it is enough to show $X_{\lambda_{1}^{\prime}}+X_{\lambda_{2}^{\prime}}$ is NBUE as $X_{\lambda_{1}^{*}}$ is exponential. This will follow from the fact that the density function of convolution of exponential random variables is logconcave which implies NBUE property (Barlow and Proschan [8]).

\section{(iii) Case 3:}

$$
\frac{1}{\lambda_{2}}<\frac{1}{\lambda_{1}^{\prime}}+\frac{1}{\lambda_{2}^{\prime}}
$$

and

$$
\frac{1}{\lambda_{1}}<\frac{1}{\lambda_{1}^{\prime}}+\frac{1}{\lambda_{2}^{\prime}}
$$

By (5.3.3), let us assume there exists an exponential random $X_{\lambda_{2}^{*}}$ which is independent of $X_{\lambda_{1}}$, where

$$
\frac{1}{\lambda_{1}}+\frac{1}{\lambda_{2}} \geq \frac{1}{\lambda_{1}}+\frac{1}{\lambda_{2}^{*}}=\frac{1}{\lambda_{1}^{\prime}}+\frac{1}{\lambda_{2}^{\prime}}
$$

Using the similar argument in Case 1, it holds that

$$
X_{\lambda_{1}}+X_{\lambda_{2}} \geq_{R S} X_{\lambda_{1}}+X_{\lambda_{2}^{*}}
$$


Hence, it is enough to show that

$$
X_{\lambda_{1}}+X_{\lambda_{2}^{*}} \geq_{R S} X_{\lambda_{1}^{\prime}}+X_{\lambda_{2}^{\prime}}
$$

So, for convenience, we can assume,

$$
\frac{1}{\lambda_{1}}+\frac{1}{\lambda_{2}}=\frac{1}{\lambda_{1}^{\prime}}+\frac{1}{\lambda_{2}^{\prime}}=c
$$

and prove

$$
X_{\lambda_{1}}+X_{\lambda_{2}} \geq_{R S} X_{\lambda_{1}^{\prime}}+X_{\lambda_{2}^{\prime}}
$$

Now, let $\lambda=\lambda_{1}$ and $\lambda^{\prime}=\lambda_{1}^{\prime}$, then $1 / \lambda_{2}=c-1 / \lambda$ and $1 / \lambda_{2}^{\prime}=c-1 / \lambda^{\prime}$. It holds that,

$$
c>\frac{1}{\lambda} \geq \frac{1}{\lambda^{\prime}} \geq \frac{c}{2}>0 .
$$

In the rest of the proof, we only discuss the case that $1 / \lambda^{\prime}>c / 2$, as the limiting argument applies when $1 / \lambda^{\prime}=c / 2$. Let $F_{\lambda}$ be the distribution function of $X_{\lambda_{1}}+X_{\lambda_{2}}$. Note that, for $t \geq 0$,

$$
\bar{F}_{\lambda}(t)=\frac{1}{\frac{2}{\lambda}-c}\left[\frac{1}{\lambda} \exp \{-\lambda t\}-\left(c-\frac{1}{\lambda}\right) \exp \left\{-\frac{t}{c-\frac{1}{\lambda}}\right\}\right] .
$$

Hence, for $t \geq 0$,

$$
\begin{aligned}
W_{\lambda}(t) & =\frac{1}{\frac{2}{\lambda}-c}\left[\frac{1}{\lambda} \int_{t}^{\infty} \exp \{-\lambda x\} d x-\left(c-\frac{1}{\lambda}\right) \int_{t}^{\infty} \exp \left\{-\frac{x}{c-\frac{1}{\lambda}}\right\} d x\right] \\
& =\frac{1}{2 \lambda-c \lambda^{2}} \exp \{-\lambda t\}-\frac{(\lambda c-1)^{2}}{2 \lambda-c \lambda^{2}} \exp \left\{-\frac{t}{c-\frac{1}{\lambda}}\right\} \\
& =\frac{\exp \{-\lambda t\}-(\lambda c-1)^{2} \exp \left\{-\frac{\lambda t}{\lambda c-1}\right\}}{2 \lambda-c \lambda^{2}} .
\end{aligned}
$$


So, taking derivative with respect to $\lambda$, for $t \geq 0$,

$$
\begin{aligned}
& W_{\lambda}^{\prime}(t)=-\frac{t \exp \{-\lambda t\}+2(\lambda c-1) c \exp \left\{-\frac{\lambda t}{\lambda c-1}\right\}+t \exp \left\{-\frac{\lambda t}{\lambda c-1}\right\}}{2 \lambda-c \lambda^{2}} \\
& -\frac{\left[\exp \{-\lambda t\}-(\lambda c-1)^{2} \exp \left\{-\frac{\lambda t}{\lambda c-1}\right\}\right](2-2 \lambda c)}{\left(2 \lambda-c \lambda^{2}\right)^{2}} .
\end{aligned}
$$

Note that, for $t \geq 0$,

$$
\begin{aligned}
& -\frac{W_{\lambda}^{\prime}(t)}{\bar{F}_{\lambda}(t)} \\
\propto & \frac{t \exp \{-\lambda t\}\left(2 \lambda-c \lambda^{2}\right)+[2(\lambda c-1) c+t]\left(2 \lambda-c \lambda^{2}\right) \exp \left\{-\frac{\lambda t}{\lambda c-1}\right\}}{\exp \{-\lambda t\}-(\lambda c-1) \exp \left\{-\frac{\lambda t}{\lambda c-1}\right\}} \\
& +\frac{\left[\exp \{-\lambda t\}-(\lambda c-1)^{2} \exp \left\{-\frac{\lambda t}{\lambda c-1}\right\}\right](2-2 \lambda c)}{\exp \{-\lambda t\}-(\lambda c-1) \exp \left\{-\frac{\lambda t}{\lambda c-1}\right\}} \\
= & \frac{(2-\lambda c) \lambda t\left[1+\exp \left\{-\lambda t\left(\frac{2-\lambda c}{\lambda c-1}\right)\right\}\right]-2(\lambda c-1)\left[1-\exp \left\{-\lambda t\left(\frac{2-\lambda c}{\lambda c-1}\right)\right\}\right]}{1-(\lambda c-1) \exp \left\{-\lambda t\left(\frac{2-\lambda c}{\lambda c-1}\right)\right\}} \\
= & \frac{(2-\lambda c) \lambda t\left[1+\exp \left\{-\lambda t\left(\frac{2-\lambda c}{\lambda c-1}\right)\right\}\right]-2\left[1-(\lambda c-1) \exp \left\{-\lambda t\left(\frac{2-\lambda c}{\lambda c-1}\right)\right\}\right]}{1-(\lambda c-1) \exp \left\{-\lambda t\left(\frac{2-\lambda c}{\lambda c-1}\right)\right\}} \\
+ & \frac{2(2-\lambda c)}{1-(\lambda c-1) \exp \left\{-\lambda t\left(\frac{2-\lambda c}{\lambda c-1}\right)\right\}},
\end{aligned}
$$

by Lemma 5.3.6, it is enough to show,

$$
h(t)=\frac{\lambda t\left[1+\exp \left\{-\lambda t\left(\frac{2-\lambda c}{\lambda c-1}\right)\right\}\right]+2}{1-(\lambda c-1) \exp \left\{-\lambda t\left(\frac{2-\lambda c}{\lambda c-1}\right)\right\}},
$$

is increasing in $t \geq 0$. Denote

$$
\gamma=\frac{2-\lambda c}{\lambda c-1}>0
$$

Thus,

$$
h(t)=\frac{\lambda t\left(1+e^{-\lambda t \gamma}\right)+2}{1-\frac{1}{\gamma+1} e^{-\lambda t \gamma}} .
$$


Taking derivative with respect to $t$, it holds that,

$$
h^{\prime}(t)=\frac{\lambda\left(1+e^{-\lambda t \gamma}\right)-\lambda^{2} t \gamma e^{-\lambda t \gamma}}{1-\frac{1}{\gamma+1} e^{-\lambda t \gamma}}-\frac{\lambda t\left(1+e^{-\lambda t \gamma}\right)+2}{\left(1-\frac{1}{\gamma+1} e^{-\lambda t \gamma}\right)^{2}} \frac{\lambda \gamma}{\gamma+1} e^{-\lambda t \gamma} .
$$

Hence, $h^{\prime}(t) \geq 0$ is equivalent to, for $t \geq 0$,

$$
\begin{aligned}
& \left(1+e^{-\lambda t \gamma}-\lambda t \gamma e^{-\lambda t \gamma}\right)\left(1-\frac{1}{\gamma+1} e^{-\lambda t \gamma}\right) \\
& \geq \frac{\gamma}{\gamma+1} e^{-\lambda t \gamma}\left[2+\lambda t\left(1+e^{-\lambda t \gamma}\right)\right]
\end{aligned}
$$

i.e.,

$$
\left(1+e^{-\lambda t \gamma}-\lambda t \gamma e^{-\lambda t \gamma}\right)\left(\frac{\gamma+1}{\gamma} e^{\lambda t \gamma}-\frac{1}{\gamma}\right) \geq 2+\lambda t\left(1+e^{-\lambda t \gamma}\right)
$$

which could be further simplified as, for $t \geq 0$,

$$
e^{\lambda t \gamma}+\frac{1}{\gamma}\left(e^{\lambda t \gamma}-e^{-\lambda t \gamma}\right) \geq 1+(\gamma+2) \lambda t
$$

Observing that, for $t \geq 0$,

$$
\sinh (\lambda t \gamma)=\frac{e^{\lambda t \gamma}-e^{-\lambda t \gamma}}{2} \geq \lambda t \gamma
$$

and

$$
e^{\lambda t \gamma} \geq 1+\lambda t \gamma
$$

the inequality (5.2.5) is true.

"Necessity $\Longleftarrow "$. Observing that the convolution of exponential distributions is IFR (increasing failure rate) which implies DMRL (Barlow and Proschan [8]), according to Theorem 3.C.5 of Shaked and Shanthikumar [60], it holds that

$$
X_{\lambda_{1}}+X_{\lambda_{2}} \geq_{R S} X_{\lambda_{1}^{\prime}}+X_{\lambda_{2}^{\prime}} \Rightarrow X_{\lambda_{1}}+X_{\lambda_{2}} \geq_{m r l} X_{\lambda_{1}^{\prime}}+X_{\lambda_{2}^{\prime}} .
$$


The required result follows from Theorem 3.1 of Zhao and Balakrishnan [68].

Using the similar argument in Theorem 1 of Bon and Păltănea [13] or Theorem 4.1 in Zhao and Balakrishnan [68], one could easily extend Theorem 5.3.3 to the case of $n \geq 3$. The proof is omitted for briefness.

Theorem 5.2.3 Let $X_{1}, \cdots, X_{n}$ be independent exponential random variables with $X_{i}$ having hazard rate $\lambda_{i}$ and let $Y_{1}, \cdots, Y_{n}$ be independent exponential random variables with $Y_{i}$ having hazard rate $\lambda_{i}^{\prime}$, for $i=1, \cdots, n$. Then,

$$
\left(\lambda_{1}, \cdots, \lambda_{n}\right) \succeq\left(\lambda_{1}^{\prime}, \cdots, \lambda_{n}^{\prime}\right) \Longrightarrow \sum_{i=1}^{n} X_{i} \geq_{R S} \sum_{i=1}^{n} Y_{i}
$$

Note that the NBUE order is equivalent to the right spread order with the same mean. Observing that

$$
\left(1 / \lambda_{1}, \cdots, 1 / \lambda_{n}\right) \succeq_{\mathrm{m}}\left(1 / \lambda_{1}^{\prime}, \cdots, 1 / \lambda_{n}^{\prime}\right) \Longrightarrow\left(\lambda_{1}, \cdots, \lambda_{n}\right) \underbrace{\mathrm{rm}}\left(\lambda_{1}^{\prime}, \cdots, \lambda_{n}^{\prime}\right),
$$

the following result is a direct consequence of Theorem 5.2.3.

Corollary 5.2.4 Let $X_{1}, \cdots, X_{n}$ be independent exponential random variables with $X_{i}$ having hazard rate $\lambda_{i}$ and let $Y_{1}, \cdots, Y_{n}$ be independent exponential random variables with $Y_{i}$ having hazard rate $\lambda_{i}^{\prime}$, for $i=1, \cdots, n$. Then,

$$
\left(1 / \lambda_{1}, \cdots, 1 / \lambda_{n}\right) \succeq_{\mathrm{m}}\left(1 / \lambda_{1}^{\prime}, \cdots, 1 / \lambda_{n}^{\prime}\right) \Longrightarrow \sum_{i=1}^{n} X_{i} \geq_{\mathrm{NBUE}} \sum_{i=1}^{n} Y_{i}
$$


The following result is a direct consequence of Corollary 5.3.8 as the NBUE order implies the Lorenz order, which is of great interest in economics.

Corollary 5.2.5 Let $X_{1}, \cdots, X_{n}$ be independent exponential random variables with $X_{i}$ having hazard rate $\lambda_{i}$ and let $Y_{1}, \cdots, Y_{n}$ be independent exponential random variables with $Y_{i}$ having hazard rate $\lambda_{i}^{\prime}$ for $i=1, \cdots, n$. Then,

$$
\left(1 / \lambda_{1}, \cdots, 1 / \lambda_{n}\right) \succeq_{\mathrm{m}}\left(1 / \lambda_{1}^{\prime}, \cdots, 1 / \lambda_{n}^{\prime}\right) \Longrightarrow \sum_{i=1}^{n} X_{i} \geq_{\text {Lorenz }} \sum_{i=1}^{n} Y_{i}
$$

The NBUE order can be used to compare the shapes of distributions. The following theorem reveals that the density function of convolution of heterogeneous exponential random variables is more skewed than that of homogeneous exponential random variables.

Corollary 5.2.6 Let $X_{1}, \cdots, X_{n}$ be independent exponential random variables with $X_{i}$ having hazard rate $\lambda_{i}$ for $i=1, \cdots, n$ and $Z_{1}, \cdots, Z_{n}$ be independent and identically distributed exponential random variables. Then,

$$
\sum_{i=1}^{n} X_{i} \geq_{\mathrm{NBUE}} \sum_{i=1}^{n} Z_{i}
$$

Proof: Let $Y_{1}, \cdots, Y_{n}$ be independent and identical exponential random variables with the same hazard rate $\lambda^{\prime}$, where

$$
\left(1 / \lambda_{1}, \cdots, 1 / \lambda_{n}\right) \succeq_{\mathrm{m}}\left(1 / \lambda^{\prime}, \cdots, 1 / \lambda^{\prime}\right)
$$


According to Corollary 5.3.8,

$$
\sum_{i=1}^{n} X_{i} \geq_{\mathrm{NBUE}} \sum_{i=1}^{n} Y_{i}
$$

Note that $\sum_{i=1}^{n} Y_{i}$ is a gamma random variable with shape parameter $n$ and scale parameter $1 / \lambda^{\prime}$. Sine the NBUE order is scale invariant, it follows that

$$
\sum_{i=1}^{n} X_{i} \geq_{\mathrm{NBUE}} \sum_{i=1}^{n} Y_{i}=\mathrm{NBUE} \sum_{i=1}^{n} Z_{i} .
$$

Hence, the required result follows immediately.

Figure 1 gives an illustration of the above result. The highest peak curve is the density function with $\left(\lambda_{1}, \lambda_{2}\right)=(1 / 2,1 / 2)$; the middle peak curve is the density function with $\left(\lambda_{1}, \lambda_{2}\right)=(1 / 0.2,1 / 3.8)$; the lowest peak curve is the density function with $\left(\lambda_{1}, \lambda_{2}\right)=(1 / 1.5,1 / 2.5)$. It is seen that

$$
(0.2,3.8) \succeq_{\mathrm{m}}(1.5,2.5) \succeq_{\mathrm{m}}(2,2)
$$

The following result gives an equivalent characterization of right spread order between convolutions of two exponential samples when one sample has heterogeneous hazard rates but the other has homogeneous hazard rates.

Corollary 5.2.7 Let $X_{1}, \cdots, X_{n}$ be independent exponential random variables with $X_{i}$ having hazard rate $\lambda_{i}$ for $i=1, \ldots, n$ and let $Y_{1}, \cdots, Y_{n}$ be independent and identical exponential random variables with the same hazard rate $\lambda$. Then,

$$
\sum_{i=1}^{n} X_{i} \geq_{R S} \sum_{i=1}^{n} Y_{i} \Longleftrightarrow \mathrm{E}\left(\sum_{i=1}^{n} X_{i}\right) \geq \mathrm{E}\left(\sum_{i=1}^{n} Y_{i}\right) .
$$




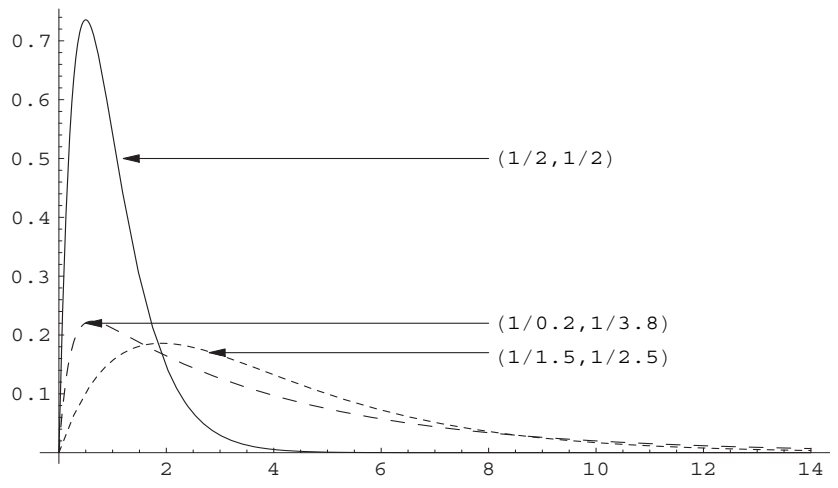

Figure 5.1: The density curves with different parameters.

Proof: Theorem 4.3 in Fernández-Ponce et al. [20] shows $X \geq$ NBUE $Y$ and $\mathrm{E} X \geq \mathrm{E} Y$ imply $X \geq_{R S} Y$. Since $X \geq_{R S} Y$ implies $\mathrm{E} X \geq \mathrm{E} Y$ when $X$ and $Y$ have the same left support, the result follows from Corollary 5.2.6.

As the convolution of exponential distributions is DMRL (Barlow and Proschan [8]), using Theorem 3.C.5 of Shaked and Shanthikumar [60], one can easily derive the following result, which has been reported by Zhao and Balakrishnan [68].

Corollary 5.2.8 Let $X_{1}, \cdots, X_{n}$ be independent exponential random variables such that $X_{i}$ has hazard rate $\lambda_{i}, i=1, \ldots, n$ and let $Y_{1}, \cdots, Y_{n}$ be independent exponential random variables with $Y_{i}$ having hazard rate $\lambda_{i}^{\prime}$, for $i=1, \cdots, n$. Then,

$$
\left(\lambda_{1}, \cdots, \lambda_{n}\right) \stackrel{\mathrm{rm}}{\succeq}\left(\lambda_{1}^{\prime}, \cdots, \lambda_{n}^{\prime}\right) \Longrightarrow \sum_{i=1}^{n} X_{i} \geq_{m r l} \sum_{i=1}^{n} Y_{i}
$$




\subsection{Tail behavior of the convolutions of gamma random variables}

\subsubsection{Introduction}

The gamma distribution is one of the most popular distributions in statistics, engineering and reliability applications. In particular, gamma distribution plays a prominent role in actuarial science since most total insurance claim distributions have roughly the same shape as gamma distributions: skewed to the right, nonnegatively supported and unimodal (cf. Furman [21]). As it is well-known, the gamma distribution includes exponential and chi-square, two important distributions, as special cases. Due to the complicated distribution function of gamma random variable, most of the work in the literature discussed only the convolutions of exponential random variables. Some relevant references are Boland, et al. [11], Kochar and Ma [35], Bon and Păltănea [13], Zhao and Balakrishnan [68], Bock, et al. [9] and Diaconis and Perlman [17] studied convolutions of gamma random variables.

Let $X_{1}, \ldots, X_{n}$ be a random sample from a gamma distribution with shape parameter $a>0$, scale parameter $\lambda>0$ and with density function

$$
f(x)=\frac{\lambda^{a}}{\Gamma(a)} x^{a-1} \exp \{-\lambda x\}, \quad x \geq 0 .
$$

We are interested in studying the stochastic properties of statistics of the form

$$
W=\theta_{1} X_{1}+\theta_{2} X_{2}+\ldots+\theta_{n} X_{n},
$$


were $\theta_{1}, \ldots, \theta_{n}$ are positive weights (constants). Bock, et al. [9] showed that for $n=2$, if

$$
t \leq \frac{a\left(\theta_{1}+\theta_{2}\right)}{\lambda}
$$

then $P(W \leq t)$ is Schur-convex in $\left(\theta_{1}, \theta_{2}\right)$; and if

$$
t \geq \frac{(a+1 / 2)\left(\theta_{1}+\theta_{2}\right)}{\lambda}
$$

then $P(W \geq t)$ is Schur-convex in $\left(\theta_{1}, \theta_{2}\right)$. For general $n>2$, then $P(W \leq t)$ is Schur-convex in the region

$$
\left\{\theta: \min _{1 \leq i \leq n} \theta_{i} \geq \frac{t \lambda}{n a+1}\right\}
$$

and $P(W \geq t)$ is Schur-convex in $\theta$ for

$$
t \geq \frac{(n a+1)\left(\theta_{1}+\theta_{2}+\ldots+\theta_{n}\right)}{\lambda}
$$

Diaconis and Perlman [17] further studied the tail probabilities of convolution of gamma random variables. They pointed out that if

$$
\left(\theta_{1}, \cdots, \theta_{n}\right) \succeq_{\mathrm{m}}\left(\theta_{1}^{\prime}, \cdots, \theta_{n}^{\prime}\right)
$$

then

$$
\operatorname{Var}\left(\sum_{i=1}^{n} \theta_{i} X_{i}\right) \geq \operatorname{Var}\left(\sum_{i=1}^{n} \theta_{i}^{\prime} X_{i}\right) .
$$

This property states that if the weights are more dispersed in the sense of majorization, then the convolutions are more dispersed about their means as measured by their variances. Diaconis and Perlman [17] also wondered whether 
$\sum_{i=1}^{n} \theta_{i} X_{i}$ is more dispersed than $\sum_{i=1}^{n} \theta_{i}^{\prime} X_{i}$ as measured by the stronger criterion of their tail probabilities. They tried to answer this question by proving that under the condition (5.3.1), the distribution functions of $\sum_{i=1}^{n} \theta_{i} X_{i}$ and $\sum_{i=1}^{n} \theta_{i}^{\prime} X_{i}$ have only one crossing. However, they only proved this result for $n=2$. For $n \geq 3$, they required further restrictions. Hence, this problem has been open for a long time, which is also known as Unique Crossing Conjecture, or UCC.

It is possible to investigate this problem in other metrics for the $\theta_{i}$ 's. For instance, if $X_{\lambda_{i}}$ are independent gamma random variables with a common shape parameter $a$ and scale parameters $\lambda_{i}$ and $X_{\lambda_{i}^{\prime}}$ are independent gamma random variables with a common shape parameter $a$ and scale parameters $\lambda_{i}^{\prime}$, for $i=$ $1, \cdots, n$, Korwar [43] showed that under the condition of majorization order, for $a \geq 1$

$$
\left(\lambda_{1}, \cdots, \lambda_{n}\right) \succeq_{\mathrm{m}}\left(\lambda_{1}^{\prime}, \cdots, \lambda_{n}^{\prime}\right) \Longrightarrow \sum_{i=1}^{n} X_{\lambda_{i}} \geq_{\text {disp }} \sum_{i=1}^{n} X_{\lambda_{i}^{\prime}} .
$$

Khaledi and Kochar [30] relaxed the condition by proving that under the $p$-larger order, which is a weaker order than the majorization order, for $a \geq 1$,

$$
\left(\lambda_{1}, \cdots, \lambda_{n}\right) \succeq\left(\lambda_{1}^{\prime}, \cdots, \lambda_{n}^{\prime}\right) \Longrightarrow \sum_{i=1}^{n} X_{\lambda_{i}} \geq_{\text {disp }} \sum_{i=1}^{n} X_{\lambda_{i}^{\prime}} .
$$

In this Chapter, I will further investigate the problem posed by Diaconis and Perlman [17]. For the case of $n=2$, I will strengthen the single crossing property to the star order. I also give a different sufficient condition for the star order to hold between two convolutions of scaled gamma random variables. It will be shown 
that

$$
\left(\lambda_{1}, \lambda_{2}\right) \succeq_{\mathrm{m}}\left(\lambda_{1}^{\prime}, \lambda_{2}^{\prime}\right) \Longrightarrow X_{\lambda_{1}}+X_{\lambda_{2}} \geq_{\star} X_{\lambda_{1}^{\prime}}+X_{\lambda_{2}^{\prime}}
$$

and

$$
\left(\theta_{1}, \theta_{2}\right) \succeq_{\mathrm{m}}\left(\theta_{1}^{\prime}, \theta_{2}^{\prime}\right) \Longrightarrow \theta_{1} X_{1}+\theta_{2} X_{2} \geq_{\star} \theta_{1}^{\prime} X_{1}+\theta_{2}^{\prime} X_{2}
$$

where $X_{1}$ and $X_{2}$ are independent and identically distributed gamma random variables. Note that (5.3.2) could be equivalently expressed as

$$
\left(1 / \lambda_{1}, 1 / \lambda_{2}\right) \succeq_{\mathrm{m}}\left(1 / \lambda_{1}^{\prime}, 1 / \lambda_{2}^{\prime}\right) \Longrightarrow X_{\lambda_{1}}+X_{\lambda_{2}} \geq_{\star} X_{\lambda_{1}^{\prime}}+X_{\lambda_{2}^{\prime}}
$$

As is well-known, the star order compares the skewness of two distributions, and if $X$ is less than $Y$ according to the star order, then the survival function of $X$ crosses the survival function of $G$ at most once, and only from above, no matter how $Y$ is scaled (cf. Marshall and Olkin [48], p. 73).

Intuitively, if the scale parameters are more dispersed, then the density function of the convolution is more skewed. Actually, a special case, Theorem 4 in $\mathrm{Yu}$ [65] shows that if $X_{\lambda_{i}}$ are independent gamma random variables with a common shape parameter $a$ and scale parameters $\lambda_{i}$ and $Y_{i}$ are independent gamma random variables with common shape parameter $a$ and scale parameter $\lambda$, i.e., $Y_{i}$ are from a homogeneous sample, for $i=1, \cdots, n$, then,

$$
\sum_{i=1}^{n} X_{\lambda_{i}} \geq_{\star} \sum_{i=1}^{n} Y_{i} .
$$


Clearly, the homogeneous gamma distribution has smaller skewness in the sense of star ordering. It is noted that if in addition

$$
\mathrm{E}\left(\sum_{i=1}^{n} X_{\lambda_{i}}\right)=\mathrm{E}\left(\sum_{i=1}^{n} Y_{i}\right),
$$

then the survival function of $\sum_{i=1}^{n} Y_{i}$ crosses the survival function of $\sum_{i=1}^{n} X_{\lambda_{i}}$ exactly once and from above, which means the tail probability of $\sum_{i=1}^{n} X_{\lambda_{i}}$ is greater than that of $\sum_{i=1}^{n} Y_{i}$. In actuarial science, it may mean that the heterogeneous gamma sample has more risk.

To measure the tail risk or dispersion, one good measure would be the right spread transform, which was independently proposed by Fernández-Ponce, et al. [20] and Shaked and Shanthikumar [59]. For a random variable $X$ with distribution $F$, let

$$
\begin{aligned}
W(p ; F) & =\mathrm{E}\left[\left(X-F^{-1}(p)\right)^{+}\right] \\
& =\int_{F^{-1}(p)}^{\infty} \bar{F}(x) d x,
\end{aligned}
$$

where $F^{-1}(p)=\inf \{x: F(x) \geq p\}$ for $0 \leq p \leq 1,(X)^{+}=\max \{X, 0\}$, and $\bar{F}=1-F$ is the survival function of $X$. This transform is called the right spread transform in Fernández-Ponce, et al. [20], but Shaked and Shanthikumar [59] named it as excess wealth transform since in the economics context $W(p ; F)$ can be thought of as the additional wealth of the richest $100(1-p) \%$ individuals in the population. In actuarial science, it is termed as expected shortfall to reflect the stop-loss premium with retention $F^{-1}(p)$ at probability level $p$ (see Denuit, et 
al. [16]). It is further pointed out in Sordo [62] that the right spread transform is closely related to the risk measures used in actuarial science such as tail conditional expectation, tail conditional variance, etc. More precisely, the right spread transform is related to a class of measures of the form

$$
H_{\phi, p}(X)=\mathrm{E}\left[\phi\left(X-\mathrm{E}\left(X_{p}\right)\right) \mid X>F^{-1}(p)\right]
$$

where $X_{p}=\left(X \mid X>F^{-1}(p)\right)$, and $\phi$ is a convex function. When $\phi(x)=x^{2}$, the transform reduces to the truncated variance or the tail conditional variance in insurance (cf. Furman and Lansman [22]),

$$
\mathbf{T V}_{p}(X)=\operatorname{Var}\left(X \mid X>F^{-1}(p)\right)
$$

It is shown in Sordo [62] that

$$
W(p ; F) \leq W(p ; G) \Longleftrightarrow H_{\phi, p}(X) \leq H_{\phi, p}(Y),
$$

where $G$ is the distribution function of some random variable $Y$. Hence, the right spread transform plays an important role in measuring tail risks, which is utilized in the proof of the main result in this paper. With the help of (5.3.2), we prove that, for $a \geq 1$,

$$
\left(\lambda_{1}, \cdots, \lambda_{n}\right) \stackrel{\mathrm{rm}}{\succeq}\left(\lambda_{1}^{\prime}, \cdots, \lambda_{n}^{\prime}\right) \Longrightarrow \sum_{i=1}^{n} X_{\lambda_{i}} \geq_{R S} \sum_{i=1}^{n} X_{\lambda_{i}^{\prime}}
$$

which strengthens the result in Diaconis and Perlman [17] as the " $\geq_{R S}$ " order is a stronger measure of variability than the variance. In the notation of (5.3.1) this 
result can also be rewritten as,

$$
\left(\theta_{1}, \cdots, \theta_{n}\right) \succeq_{\mathrm{w}}\left(\theta_{1}^{\prime}, \cdots, \theta_{n}^{\prime}\right) \Longrightarrow \sum_{i=1}^{n} \theta_{i} X_{i} \geq_{R S} \sum_{i=1}^{n} \theta_{i}^{\prime} X_{i}
$$

where $\succeq_{\mathrm{w}}$ means weak submajorization. This result also extends the work of Korwar [43] under a weaker constraint on the parameters.

\subsubsection{Star ordering between convolutions of gamma random variables}

Let $X_{\theta_{1}}, X_{\theta_{2}}, X_{\theta_{1}^{\prime}}$ and $X_{\theta_{2}^{\prime}}$ be independent gamma random variables with a common shape parameter $a$ and scale parameters $\theta_{1}=1 / \lambda_{1}, \theta_{2}=1 / \lambda_{2}, \theta_{1}^{\prime}=1 / \lambda_{1}^{\prime}$ and $\theta_{2}^{\prime}=1 / \lambda_{2}^{\prime}$, respectively. Proposition 2.1 of Diaconis and Perlman [17] shows that if

$$
\left(\lambda_{1}, \lambda_{2}\right) \succeq_{\mathrm{m}}\left(\lambda_{1}^{\prime}, \lambda_{2}^{\prime}\right)
$$

then the distribution of $X_{\theta_{1}}+X_{\theta_{2}}$ crosses the distribution of $X_{\theta_{1}^{\prime}}+X_{\theta_{2}^{\prime}}$ exactly once.

In this section, it will be shown that under various conditions on the scale parameters, one can establish star ordering between convolutions of gamma random variables . We need the following lemma, which is originally due to Saunders and Moran ([51], p. 429).

Lemma 5.3.1 Let $\left\{F_{\lambda} \mid \lambda \in R\right\}$ be a class of distribution functions, such that $F_{\lambda}$ is supported on some interval $(a, b) \subseteq(0, \infty)$ and has a density $f_{\lambda}$ which does not 
vanish on any subinterval of $(a, b)$. Then,

$$
F_{\lambda} \leq_{\star} F_{\lambda^{*}}, \quad \lambda \leq \lambda^{*},
$$

if and only if

$$
\frac{F_{\lambda}^{\prime}(x)}{x f_{\lambda}(x)} \text { is decreasing in } x
$$

where $F_{\lambda}^{\prime}$ is the derivative of $F_{\lambda}$ with respect to $\lambda$.

Lemma 5.3.2 (Karlin [26]) Suppose $\lambda, x, \zeta$ traverse the ordered sets $\Gamma, X$ and $Z$, respectively and if the functions $f(\lambda, x, \zeta) \geq 0$ is $\mathrm{TP}_{2}$ in each pairs of variables when the third variable is fixed, then the function

$$
h(x, \lambda)=\int_{Z} f(\lambda, x, \zeta) d \mu(\zeta)
$$

defined on $X \times \Gamma$ is $\mathrm{TP}_{2}$ in $(x, \lambda)$.

Now, we are ready to present the following result.

Theorem 5.3.3 Let $X_{\lambda_{1}}, X_{\lambda_{2}}, X_{\lambda_{1}^{\prime}}, X_{\lambda_{2}^{\prime}}$ be independent gamma random variables with a common shape parameter $a$ and scale parameters $\lambda_{1}, \lambda_{2}, \lambda_{1}^{\prime}, \lambda_{2}^{\prime}$, respectively. Then,

$$
\left(\lambda_{1}, \lambda_{2}\right) \succeq_{\mathrm{m}}\left(\lambda_{1}^{\prime}, \lambda_{2}^{\prime}\right) \Longrightarrow X_{\lambda_{1}}+X_{\lambda_{2}} \geq_{\star} X_{\lambda_{1}^{\prime}}+X_{\lambda_{2}^{\prime}}
$$


Proof: We only give the proof for the case $\lambda_{1}^{\prime} \neq \lambda_{2}^{\prime}$ as the limiting arguments can be applied to prove the desired result when $\lambda_{1}^{\prime}=\lambda_{2}^{\prime}$. Rewrite $X_{\lambda_{1}}+X_{\lambda_{2}}$ as $X_{\lambda}+X_{c-\lambda}$, where $\lambda \in(c / 2, c]$. Similarly, $X_{\lambda_{1}^{\prime}}+X_{\lambda_{2}^{\prime}}$ could be rewritten as $X_{\lambda^{\prime}}+X_{c-\lambda^{\prime}}$, where $\lambda^{\prime} \in(c / 2, c]$. Then, $\left(\lambda_{1}, \lambda_{2}\right) \succeq_{\mathrm{m}}\left(\lambda_{1}^{\prime}, \lambda_{2}^{\prime}\right)$ implies $\lambda \geq \lambda^{\prime}$.

Let $F_{\lambda}(\cdot ; a)$ and $f_{\lambda}(\cdot ; a)$ be the distribution function and density function of $X_{\lambda}+X_{c-\lambda}$. From equation (3.4) of Kowar [43],

$$
F_{\lambda}^{\prime}(t ; a)=\frac{c-2 \lambda}{2 a \lambda^{2}(c-\lambda)^{2}}\left\{c a f_{\lambda}(t ; a+1)+\lambda(c-\lambda) t f_{\lambda}(t ; a)\right\}
$$

So,

$$
-\frac{F_{\lambda}^{\prime}(t ; a)}{t f_{\lambda}(t ; a)} \propto \frac{c a f_{\lambda}(t ; a+1)}{t f_{\lambda}(t ; a)}+\lambda(c-\lambda) .
$$

Hence, according to Lemma 5.3.1,

$$
X_{\lambda}+X_{c-\lambda} \geq_{\star} X_{\lambda^{\prime}}+X_{c-\lambda^{\prime}}
$$

is equivalent that,

$$
\frac{f_{\lambda}(t ; a+1)}{t f_{\lambda}(t ; a)}
$$

is increasing in $t \in(0, \infty)$. It is seen from Kowar [43], for $c / 2<\lambda \leq c$,

$$
f_{\lambda}(t ; a)=\sqrt{\pi} \frac{[\lambda(c-\lambda)]^{a}}{\Gamma(a)}\left(\frac{t}{2 \lambda-c}\right)^{a-1 / 2} \exp \left(-\frac{c t}{2}\right) I_{a-1 / 2}\left(\left(\lambda-\frac{c}{2}\right) t\right)
$$

where

$$
I_{a-1 / 2}(t)=\frac{2(t / 2)^{a-1 / 2}}{\sqrt{\pi} \Gamma(a)} \int_{0}^{1}\left(1-y^{2}\right)^{a-1} \cosh (t y) d y .
$$


Thus, it is enough to show

$$
\begin{aligned}
\frac{I_{(a+1)-1 / 2}(t)}{I_{a-1 / 2}(t)} \propto \frac{\int_{0}^{1}\left(1-y^{2}\right)^{a} t \cosh (t y) d y}{\int_{0}^{1}\left(1-y^{2}\right)^{a-1} \cosh (t y) d y} \\
=\frac{2 a \int_{0}^{1}\left(1-y^{2}\right)^{a-1} y \sinh (t y) d y}{\int_{0}^{1}\left(1-y^{2}\right)^{a-1} \cosh (t y) d y}
\end{aligned}
$$

is increasing in $t \in(0, \infty)$, where the equality follows from the integration by parts. Let

$$
h(i, t)=\int_{0}^{1} f(i, y, t) d y
$$

for $i \times t \in\{1,2\} \times(0, \infty)$, where

$$
\begin{gathered}
f(2, y, t)=\left(1-y^{2}\right)^{a-1} y \sinh (t y), \\
f(1, y, t)=\left(1-y^{2}\right)^{a-1} \cosh (t y) .
\end{gathered}
$$

Note that,

$$
\frac{f(2, y, t)}{f(1, y, t)}=y \tanh (t y)
$$

is increasing in $t>0$ and $y \in(0,1)$, as $\tanh (\cdot)$ is an increasing function. So, $f(i, y, t)$ is $\mathrm{TP}_{2}$ in both $(i, y)$ and $(i, t)$. It has been proved in Kowar ([26] p. 352) that $\cosh (t y)$ is $\mathrm{TP}_{2}$ in $(t, y) \in(0, \infty) \times(0, \infty)$. Hence, $f(1, y, t)$ is $\mathrm{TP}_{2}$ in $(t, y) \in(0, \infty) \times(0,1)$. Since

$$
\sinh (t y)=\int_{0}^{\infty} \mathrm{I}(x \leq y) t \cosh (t x) d x
$$


$\mathrm{I}(x \leq y)$ is $\mathrm{TP}_{2}$ in $(x, y) \in(0, \infty) \times(0, \infty)$ and $t \cosh (t x)$ is $\mathrm{TP}_{2}$ in $(t, x) \in$ $(0, \infty) \times(0, \infty)$, using basic composition formula of Karlin ([26], p. 17), it holds that $\sinh (t y)$ is $\mathrm{TP}_{2}$ in $(t, y) \in(0, \infty) \times(0, \infty)$. Thus, $f(2, y, t)$ is $\mathrm{TP}_{2}$ in $(t, y) \in$ $(0, \infty) \times(0,1)$

Now, using Lemma 5.3.2, $h(i, t)$ is $\mathrm{TP}_{2}$ for $i \times t \in\{1,2\} \times(0, \infty)$. Hence, the required result follows immediately.

One may wonder whether Theorem 5.3.3 can be extended to the general case of $n \geq 3$. Unfortunately, the following example gives a negative answer.

Example 5.3.4 Let $X_{\lambda}$ denote a gamma random variable with a shape parameter $a=1$ (i.e., exponential random variable) and scale parameter $\lambda$. Now, let $Y_{1}=$ $X_{0.2}+X_{1}+X_{9}$ and $Y_{2}=X_{0.2}+X_{4}+X_{6}$, where the random variables are independent. It is seen that,

$$
(0.2,1,9) \succeq_{\mathrm{m}}(0.2,4,6)
$$

Note that $E\left(Y_{1}\right)=1 / 0.2+1+1 / 9=6.111$ and $\operatorname{Var}\left(Y_{1}\right)=1 / 0.04+1+1 / 81=$ 26.012. Hence, its coefficient of variation is,

$$
\gamma_{Y_{1}}=\frac{\sqrt{26.012}}{6.111} \approx 0.835
$$

Similarly, $E\left(Y_{2}\right)=1 / 0.2+1 / 4+1 / 6=5.417, \operatorname{Var}\left(Y_{2}\right)=1 / 0.04+1 / 16+1 / 36=$ 25.090 and the coefficient of variation of $Y_{2}$ is

$$
\gamma_{Y_{2}}=\frac{\sqrt{25.090}}{5.417} \approx 0.925
$$


It is seen that

$$
\gamma_{Y_{2}}>\gamma_{Y_{1}}
$$

which invalidates $Y_{1} \geq_{\star} Y_{2}$.

The following example shows that Theorem 5.3.3 does not hold for when the shape parameters are different.

Example 5.3.5 Let $X_{a, \lambda}$ denote a gamma random variable with a shape parameter $a$ and scale parameter $\lambda$. Now, let $Y_{1}=X_{50,1}+X_{2,10}$ and $Y_{2}=X_{2,3}+X_{50,8}$, where the random variables are independent. It is seen that,

$$
(1,10) \succeq_{\mathrm{m}}(3,8)
$$

Note that the coefficient of variation of $Y_{2}$ is

$$
\gamma_{Y_{2}}=\frac{\sqrt{2 / 3^{2}+50 / 8^{2}}}{2 / 3+50 / 8} \approx 0.145
$$

and the coefficient of variation of $Y_{1}$ is

$$
\gamma_{Y_{1}}=\frac{\sqrt{2 / 10^{2}+50}}{2 / 10+50} \approx 0.141 .
$$

Since

$$
\gamma_{Y_{2}}>\gamma_{Y_{1}}
$$

$Y_{1} \geq_{\star} Y_{2}$ can not hold. 
The next result gives a different sufficient condition on the scale parameters of the convoluting gamma random variables for star ordering to hold.

Theorem 5.3.6 Let $X_{\theta_{1}}, X_{\theta_{2}}, X_{\theta_{1}^{\prime}}, X_{\theta_{2}^{\prime}}$ be independent gamma random variables with a common shape parameter $a$ and scale parameters $\theta_{1}=1 / \lambda_{1}, \theta_{2}=1 / \lambda_{2}$, $\theta_{1}^{\prime}=1 / \lambda_{1}^{\prime}$ and $\theta_{2}^{\prime}=1 / \lambda_{2}^{\prime}$. Then,

$$
\left(\lambda_{1}, \lambda_{2}\right) \succeq_{\mathrm{m}}\left(\lambda_{1}^{\prime}, \lambda_{2}^{\prime}\right) \Longrightarrow X_{\theta_{1}}+X_{\theta_{2}} \geq_{\star} X_{\theta_{1}^{\prime}}+X_{\theta_{2}^{\prime}}
$$

Proof: Similar to Theorem 5.3.3, we only give the proof for the case $\lambda_{1}^{\prime} \neq \lambda_{2}^{\prime}$ as the limiting argument applies when $\lambda_{1}^{\prime}=\lambda_{2}^{\prime}$. Without loss of generality, assume

$$
\lambda_{1}+\lambda_{2}=\lambda_{1}^{\prime}+\lambda_{2}^{\prime}=1,
$$

and let $\lambda_{1}=\lambda>\lambda_{2}$, and $\lambda_{1}^{\prime}=\lambda^{\prime}>\lambda_{2}$. Then,

$$
\left(\lambda_{1}, \lambda_{2}\right) \succeq_{\mathrm{m}}\left(\lambda_{1}^{\prime}, \lambda_{2}^{\prime}\right)
$$

is equivalent to $\lambda \geq \lambda^{\prime}$. Hence, $X_{\theta_{1}}+X_{\theta_{2}}$ can be equivalently expressed as $\lambda X_{1}+(1-\lambda) X_{2}$, where $X_{1}$ and $X_{2}$ are standard gamma random variables, $1 / 2<$ $\lambda<1$.

Note that, the distribution function of $\lambda X_{1}+(1-\lambda) X_{2}$ is

$$
F_{\lambda}(t)=\iint \frac{x_{1}^{a-1} e^{-x_{1}}}{\Gamma(a)} \frac{x_{2}^{a-1} e^{-x_{2}}}{\Gamma(a)} d x_{2} d x_{1}
$$


where the integration is over the region $x_{1}, x_{2} \geq 0$ and $\lambda x_{1}+(1-\lambda) x_{2} \leq t$. That is,

$$
F_{\lambda}(t)=\int_{0}^{t / \lambda} \int_{0}^{\left(t-\lambda x_{1}\right) /(1-\lambda)} \frac{x_{1}^{a} e^{-x_{1}}}{\Gamma(a)} \frac{x_{2}^{a} e^{-x_{2}}}{\Gamma(a)} d x_{2} d x_{1}
$$

Making the transforms

$$
r=x_{1}+x_{2}, \quad s=\frac{x_{1}}{x_{1}+x_{2}}
$$

it holds that,

$$
F_{\lambda}(t)=\frac{1}{\Gamma^{2}(a)} \int_{0}^{1} s^{a-1}(1-s)^{a-1} \int_{0}^{t / h(s)} r^{2 a-1} e^{-r} d r d s
$$

where

$$
h(s)=\lambda s+(1-\lambda)(1-s)
$$

Taking the derivative with respect to $\lambda$, it gives that

$$
F_{\lambda}^{\prime}(t)=\frac{t^{2 a}}{\Gamma^{2}(a)} \int_{0}^{1} \frac{(1-2 s) s^{a-1}(1-s)^{a-1}}{h^{2 a+1}(s)} e^{-t / h(s)} d s .
$$

The density function of $\lambda X_{1}+(1-\lambda) X_{2}$ could be represented as

$$
f_{\lambda}(t)=\frac{t^{2 a-1}}{\Gamma^{2}(a)} \int_{0}^{1} \frac{s^{a-1}(1-s)^{a-1}}{h^{2 a}(s)} e^{-t / h(s)} d s .
$$

Now, according to Lemma 5.3.1, it is enough to show that

$$
\frac{F_{\lambda}^{\prime}(t)}{t f_{\lambda}(t)}=\frac{\int_{0}^{1} \frac{(1-2 s) s^{a-1}(1-s)^{a-1}}{h^{2 a+1}(s)} e^{-t / h(s)} d s}{\int_{0}^{1} \frac{s^{a-1}(1-s)^{a-1}}{h^{2 a}(s)} e^{-t / h(s)} d s}
$$


is decreasing in $t$. That is,

$$
h(t, i)=\int_{0}^{1} K(s, i) L(s, t) d s
$$

is $\mathrm{TP}_{2}$ in $(t, i) \in(0, \infty) \times\{1,2\}$, where

$$
\begin{gathered}
K(s, 2)=\frac{s^{a-1}(1-s)^{a-1}}{h^{2 a}(s)}, \\
K(s, 1)=\frac{(1-2 s) s^{a-1}(1-s)^{a-1}}{h^{2 a+1}(s)},
\end{gathered}
$$

and

$$
L(s, t)=e^{-t / h(s)}
$$

From the basic composition formula of Karlin ([26], p. 17), we need to show $K(s, i)$ is $\mathrm{TP}_{2}$ in $(s, i) \in[0,1] \times\{1,2\}$, and $L(s, t)$ is $\mathrm{TP}_{2}$ in $(s, t) \in[0,1] \times(0, \infty)$.

To prove $K(s, i)$ is $\mathrm{TP}_{2}$, it is equivalent to showing

$$
v(s)=\frac{1-2 s}{h(s)}
$$

is decreasing in $s$, which turns out to be true. Hence, $K(s, i)$ is $\mathrm{TP}_{2}$ in $(s, i) \in$ $[0,1] \times\{1,2\} . L(s, t)$ is $\mathrm{TP}_{2}$, which follows from the fact $h(s)=\lambda s+(1-\lambda)(1-s)$ is strictly increasing in $s$ for $\lambda>1 / 2$.

Hence, the required result follows.

Remark: (a) Theorem 5.3.6 implies that the distribution function of $X_{\theta_{1}}+X_{\theta_{2}}$ crosses that of $X_{\theta_{1}^{\prime}}+X_{\theta_{2}^{\prime}}$ at most once, no matter how $X_{\theta_{1}}+X_{\theta_{2}}$ is scaled. As a special case, they have exactly one crossing when both sides have the same mean which strengthens Proposition 2.1 in Diaconis and Perlman [17]. 
(b) It is easy to check that the condition in Theorem 5.3.6 neither implies and nor is implied by the condition in Theorem 5.3.3.

\subsubsection{Right spread order}

With the aid of Theorem 5.3.6, I will establish the right spread ordering between two convolutions of independent gamma random variables.

Theorem 5.3.7 Let $X_{\theta_{1}}, X_{\theta_{2}}, X_{\theta_{1}^{\prime}}, X_{\theta_{2}^{\prime}}$ be independent gamma random variables

with a common shape parameter $a \geq 1$ and scale parameters $\theta_{1}, \theta_{2}, \theta_{1}^{\prime}$ and $\theta_{2}^{\prime}$. Then,

$$
\left(\theta_{1}, \theta_{2}\right) \stackrel{\mathrm{rm}}{\succeq}\left(\theta_{1}^{\prime}, \theta_{2}^{\prime}\right) \Longleftrightarrow X_{\theta_{1}}+X_{\theta_{2}} \geq_{R S} X_{\theta_{1}^{\prime}}+X_{\theta_{2}^{\prime}}
$$

Proof: "Sufficiency $\Longrightarrow$ ". Without loss of generality, assume that $\theta_{1} \leq \theta_{2}$ and $\theta_{1}^{\prime} \leq \theta_{2}^{\prime}$. As

$$
\left(\theta_{1}, \theta_{2}\right) \stackrel{\text { rm }}{\succeq}\left(\theta_{1}^{\prime}, \theta_{2}^{\prime}\right)
$$

it holds that

$$
\frac{1}{\theta_{1}} \geq \frac{1}{\theta_{1}^{\prime}}
$$

and

$$
\frac{1}{\theta_{1}}+\frac{1}{\theta_{2}} \geq \frac{1}{\theta_{1}^{\prime}}+\frac{1}{\theta_{2}^{\prime}}
$$

Case 1:

$$
\frac{1}{\theta_{2}} \geq \frac{1}{\theta_{2}^{\prime}}
$$


In this case, it holds that

$$
\frac{1}{\theta_{1}} \geq \frac{1}{\theta_{1}^{\prime}} \quad \text { and } \quad \frac{1}{\theta_{2}} \geq \frac{1}{\theta_{2}^{\prime}}
$$

Hence, it follows that

$$
\begin{aligned}
& \frac{1}{\theta_{1}} \geq \frac{1}{\theta_{1}^{\prime}} \Longrightarrow X_{\theta_{1}} \geq_{R S} X_{\theta_{1}^{\prime}}, \\
& \frac{1}{\theta_{2}} \geq \frac{1}{\theta_{2}^{\prime}} \Longrightarrow X_{\theta_{2}} \geq_{R S} X_{\theta_{2}^{\prime}} .
\end{aligned}
$$

Since $a \geq 1$ implies the density function of the gamma random variable is logconcave, using Theorem 3.C.7 of Shaked and Shanthikumar [60], it follows that,

$$
X_{\theta_{1}}+X_{\theta_{2}} \geq_{R S} X_{\theta_{1}^{\prime}}+X_{\theta_{2}^{\prime}}
$$

\section{Case 2:}

$$
\frac{1}{\theta_{2}}<\frac{1}{\theta_{2}^{\prime}}
$$

In this case, it holds that

$$
\frac{1}{\theta_{1}} \geq \frac{1}{\theta_{1}^{\prime}} \geq \frac{1}{\theta_{2}^{\prime}} \geq \frac{1}{\theta_{2}}
$$

Let us assume there exists an independent gamma random variable $X_{\theta_{1}^{*}}$, where

$$
\frac{1}{\theta_{1}} \geq \frac{1}{\theta_{1}^{*}} \geq \frac{1}{\theta_{1}^{\prime}}
$$

and

$$
\frac{1}{\theta_{1}^{*}}+\frac{1}{\theta_{2}}=\frac{1}{\theta_{1}^{\prime}}+\frac{1}{\theta_{2}^{\prime}} .
$$

It is easy to see that

$$
X_{\theta_{1}}+X_{\theta_{2}} \geq_{R S} X_{\theta_{1}^{*}}+X_{\theta_{2}},
$$


and hence it is enough to prove

$$
X_{\theta_{1}^{*}}+X_{\theta_{2}} \geq_{R S} X_{\theta_{1}^{\prime}}+X_{\theta_{2}^{\prime}}
$$

Note that, the conditions (5.3.4) and (5.3.5) are equivalent to

$$
\left(\lambda_{1}^{*}, \lambda_{2}\right) \succeq_{\mathrm{m}}\left(\lambda_{1}^{\prime}, \lambda_{2}^{\prime}\right),
$$

where $\lambda_{1}^{*}=1 / \theta_{1}^{*}, \lambda_{2}=1 / \theta_{2}, \lambda_{1}^{\prime}=1 / \theta_{1}^{\prime}$ and $\lambda_{2}^{\prime}=1 / \theta_{2}^{\prime}$. From Theorem 5.3.6, it follows that,

$$
X_{\theta_{1}^{*}}+X_{\theta_{2}} \geq_{\star} X_{\theta_{1}^{\prime}}+X_{\theta_{2}^{\prime}}
$$

which implies

$$
X_{\theta_{1}^{*}}+X_{\theta_{2}} \geq_{\mathrm{NBUE}} X_{\theta_{1}^{\prime}}+X_{\theta_{2}^{\prime}}
$$

Since both sides have the same mean, (5.3.6) is equivalent to

$$
X_{\theta_{1}^{*}}+X_{\theta_{2}} \geq_{R S} X_{\theta_{1}^{\prime}}+X_{\theta_{2}^{\prime}}
$$

Hence, the result follows.

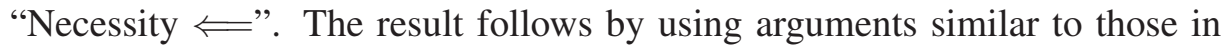
the proof of Theorem 5.2.3.

Since a gamma random variable with shape parameter $a \geq 1$ has logconcave density (Marshall and Olkin [48]), using arguments similar to those in Theorem 4.1 of Zhao and Balakrishnan [68] and Theorem 3.C.7 of Shaked and Shanthiku$\operatorname{mar}$ [60], one may extend Theorem 5.3.7 to the case of $n \geq 3$. 
Theorem 5.3.8 Let $X_{\lambda_{1}}, \ldots, X_{\lambda_{n}}$ be independent gamma random variables with a common shape parameter $a \geq 1$ and scale parameters $\lambda_{1}, \ldots, \lambda_{n}$, respectively and let $X_{\lambda_{1}^{\prime}}, \cdots, X_{\lambda_{n}^{\prime}}$ be independent gamma random variables with a common shape parameter $a \geq 1$ and scale parameters $\lambda_{1}^{\prime}, \ldots, \lambda_{n}^{\prime}$, respectively. Then,

$$
\left(\lambda_{1}, \cdots, \lambda_{n}\right) \succeq\left(\lambda_{1}^{\prime}, \cdots, \lambda_{n}^{\prime}\right) \Longrightarrow \sum_{i=1}^{n} X_{\lambda_{i}} \geq_{R S} \sum_{i=1}^{n} X_{\lambda_{i}^{\prime}}
$$

Remark: (i) Theorem 5.3.8 extends Theorem 3.3 and hence the corresponding corollaries of Theorem 5.2.3 and Zhao and Balakrishnan [68].

(ii) Theorem 2.1 in Khaledi and Kochar [30] proved the following result, for $a \geq 1$

$$
\left(\lambda_{1}, \cdots, \lambda_{n}\right) \succeq^{p}\left(\lambda_{1}^{\prime}, \cdots, \lambda_{n}^{\prime}\right) \Longrightarrow \sum_{i=1}^{n} X_{\lambda_{i}} \geq_{\text {disp }} \sum_{i=1}^{n} X_{\lambda_{i}^{\prime}} .
$$

Theorem 5.3.8 shows a parallel result on the right spread order by relaxing the condition on the scale parameters.

\subsection{Applications}

\subsubsection{Reliability theory}

Suppose a redundant standby system is composed of different exponential components (which is often a common assumption in a large system). When a component fails, one standby component is immediately put into operation. So the lifetime of the system is the convolution of the component lifetimes. Theorem 5.3.7 states that greater the degree of heterogeneity (as reflected by the reciprocal 
majorization order) among means of different components, greater is the degree of variability in the system. In practice, the engineer may only know the average lifetime of the system. Corollary 5.2.7 provides a simple lower bound on the variance of such a system.

Corollary 5.4.1 Let $X_{1}, \cdots, X_{n}$ be independent exponential components with respective hazard rates $\lambda_{i}, \ldots, \lambda_{n}$, respectively. Then,

$$
\operatorname{Var}\left(\sum_{i=1}^{n} X_{i}\right) \geq \frac{n}{\hat{\lambda}^{2}}
$$

where $\hat{\lambda}=\frac{n}{\sum_{i=1}^{n} \frac{1}{\lambda_{i}}}$ is the mean lifetime of the redundant standby system.

This bound is sharper than the one given in Kochar and Ma [35] which is in terms of the arithmetic mean of the $\lambda_{i}$ 's.

Corollary 5.2.6 has an interesting interpretation in reliability theory: a redundant standby system composed of nonidentical exponential components exhibits more "NBUE" aging property than such a system composed of homogeneous exponential components. As a further consequence, this result provides a lower bound on the coefficient of variation of the redundant standby system.

Corollary 5.4.2 Let $X_{1}, \cdots, X_{n}$ be independent exponential components with respective hazard rates $\lambda_{1}, \ldots, \lambda_{n}$. Then,

$$
c v\left(\sum_{i=1}^{n} X_{i}\right) \geq \frac{1}{\sqrt{n}} .
$$




\subsubsection{Economics}

Lorenz Curve is a graph $(x, y)$ that shows, for the bottom $100 x \%$ of the households, the percentage $100 y \%$ of the total income which they posses. If every one in the population has the same income, the bottom $p \%$ of the population would always have $p \%$ of the total income and the Lorenz curve would be the diagonal line $y=x$. The more the Lorenz Curve is below the diagonal line, the more is the disparity between the incomes. To compare the extent of inequality that exists between two incomes, the Lorenz order is used. If $X$ and $Y$ denote two incomes, $X \leq_{\text {Lorenz }} Y$ means that $X$ shows less inequality than $Y$. Now, suppose that each individual in the population has income coming from different sources (e.g. salary, stocks, bonus, etc.), which could be represented as the sum of different exponential or Gamma variables. Corollary 5.2.5 reveals that more diverse the different component distributions are, the more is the extent of inequality between the population incomes.

\subsubsection{Actuarial science}

In actuarial science, people are always interested in the following question: how much can we expect to lose with a given probability? This introduces the concept of value-at-risk (VaR), which has become the benchmark risk measure. For more 
details about VaR, please refer to Denuit et al. [16]. The VaR is defined as

$$
\operatorname{VaR}[X ; p]=F^{-1}(p)
$$

As the VaR at a fixed level only gives local information about the underlying distribution, actuaries proposed the so-called expected shortfall to overcome this shortcoming. Expected shortfall at probability level $p$ is the stop-loss premium with retention $\operatorname{VaR}[X ; p]$, that is,

$$
\begin{aligned}
\mathrm{ES}[X ; p] & =\mathrm{E}(X-\operatorname{VaR}[X ; p])_{+} \\
& =\int_{F^{-1}(p)}^{\infty} \bar{F}(x) d x
\end{aligned}
$$

where $(X)_{+}=\max \{X, 0\}$. Now, suppose that a total claim is composed of several subclaims which come from different exponential or Erlang distributions. The actuary wants to know the properties of expected shortfall in order to make a good policy for the insurance company. Theorem 5.3.7 states that greater the degree of heterogeneity among subclaims, the larger the expected shortfall is. If the actuary is able to estimate the mean of heterogeneous subclaims, Corollary 5.2.7 provides a sharp lower bound for the expected shortfall of subclaims at each probability level $p$.

For example, suppose that the total claim is composed of 3 subclaims coming from exponential distributions with parameters $\lambda_{1}, \lambda_{2}$ and $\lambda_{3}$. Then the distribution function of $X_{1}+X_{2}+X_{3}$ is

$$
F(x)=1-\frac{\lambda_{2} \lambda_{3}}{\left(\lambda_{2}-\lambda_{1}\right)\left(\lambda_{3}-\lambda_{1}\right)} e^{-\lambda_{1} x}-\frac{\lambda_{1} \lambda_{3}}{\left(\lambda_{1}-\lambda_{2}\right)\left(\lambda_{3}-\lambda_{2}\right)} e^{-\lambda_{2} x}
$$




$$
-\frac{\lambda_{1} \lambda_{2}}{\left(\lambda_{1}-\lambda_{3}\right)\left(\lambda_{2}-\lambda_{3}\right)} e^{-\lambda_{3} x}
$$

Let us assume

$$
\left(\lambda_{1}, \lambda_{2}, \lambda_{3}\right)=(1,2,3)
$$

Then, the arithmetic mean and the harmonic mean are 2 and 18/11, respectively. In Figure 2 below, we used Mathematica to plot the expected shortfalls of the total claim when the parameters are $(1,2,3)$, and their arithmetic mean and harmonic mean. It is seen that the harmonic mean provides a sharper bound for the expected shortfall as stated in Corollary 5.2.7.

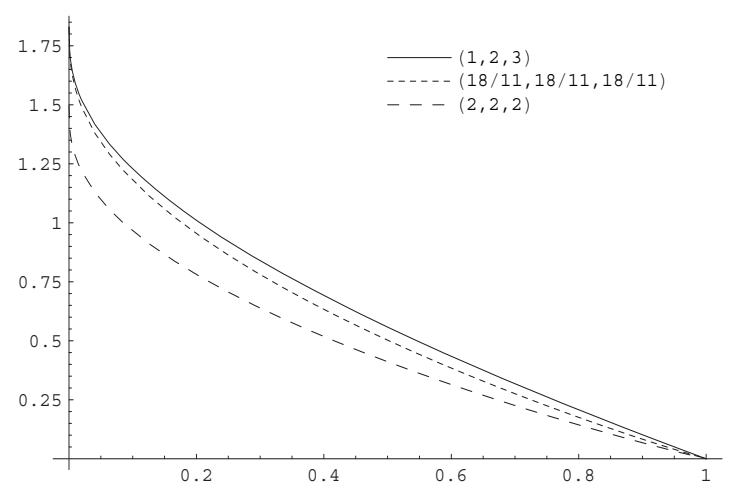

Figure 5.2: Expected shortfall of the total claim with different exponential parameters.

\subsection{Concluding remarks}

In this Chapter, I have obtained some sufficient conditions for comparing the convolutions of heterogenous exponential (Gamma) random variables. It is to be 
noted that the UCC proposed in Diaconis and Perlman [17] is still open, although Theorem 5.3.8 reveals that the more heterogeneity of gamma scale parameters implies the more tail risk of the convolutions of gamma random variables.

There are many directions in which this investigation might be continued. One possibility would be to see that whether Theorem 5.3.8 is also true for other distributions, such as Weibull and Pareto distributions. The other option would be to consider whether Theorem 5.3.6 could be generalized to the case of $n \geq 3$. One may also consider whether Theorem 5.3.7 is true for the case of $0<a<1$, as $a=1 / 2$ corresponds to the chi square random variables with one degree of freedom which is of interest in statistical inference.

Some results of this Chapter are based on Kochar and $X u$ [42]. 


\section{Bibliography}

[1] Avérous, J., Genest, C. and Kochar, S. C., (2005). On dependence structure of order statistics. J. Multivariate Anal. 94, 159-171.

[2] Ahmed, A. N., Alzaid, A., Bartoszewicz, J. and Kochar, S.C. (1986). Dispersive and superadditive ordering. Advances in Applied Probability 18, 10191022

[3] Arnold, B. C. and Groeneveld, R. A. (1995). Measuring skewness with respect to the mode. The American Statistician 49, 34-38.

[4] Balakrishnan, N. (2007). Permanents, order statistics, outliers, and robustness. Revista Matemática Complutense 20, 7-107.

[5] Balakrishnan, N. and Basu, A. P. (1995). The Exponential Distribution: Theory, Methods and Applications. Gordon and Breach, Newark, New Jersey.

[6] Balakrishnan, N. and Rao, C. R. (1998). Handbook of Statistics 16-Order Statistics: Theory and Methods. Elsevier, New York. 
[7] Balakrishnan, N. and Rao, C. R. (1998). Handbook of Statistics 17-Order Statistics: Applications. Elsevier, New York.

[8] Barlow, R. E. and Proschan, F. (1981). Statistical Theory of Reliability and Life Testing. To Begin with, Silver Spring, Maryland.

[9] Bock, M. E., Diaconis, P., Huffer, F. W. and Perlman, M. D. (1987). Inequalities for linear combinations of Gamma random variables. The Canadian Journal of Statistics 15 387-395.

[10] Boland, P. J., El-Neweihi, E. and Proschan, F. (1994). Applications of the hazard rate ordering in reliability and order statistics. J. Appl. Probab. 31, 180192.

[11] Boland, P. J., El-Neweihi, E. and Proschan, F. (1994). Schur properties of convolutions of exponential and geometric random variables. Journal of Multivariate Analysis 48, 157-167.

[12] Boland,P.J ., Hollander, M., Joag-Dev, K. and Kochar, S. C. (1996). Bivariate dependence properties of order statistics, J. Multivariate Anal. 56, 75-89.

[13] Bon, J. L. and Păltănea, E. (1999). Ordering properties of convolutions of exponential random variables. Lifetime Data Anal. 5, 185-192. 
[14] Bon, J.L. and Păltănea, E. (2006). Comparison of order statistics in a random sequence to the same statistics with i.i.d. variables. ESAIM: Probability and Statistics 10, 1-10.

[15] David, H. A. and Nagaraja, H.N. (2003). Order statistics. 3rd ed. Wiley, New York.

[16] Denuit, M., Dhaene, J., Goovaerts, M. and Kaas, R. Actuarial Theory for Dependent Risks: Measures, Orders and Models. John Wiley \& Sons, 2005.

[17] Diaconis, P. and Perlman, M. D. Bounds for tail probabilities of linear combinations of independent gamma random variables. The Symposium on dependence in Statistics and Probability, Hidden Valley, Pennsylvania, 1987.

[18] Dolati, A., Genest, C. and Kochar, S. C. (2008). On the dependence between the extreme order statistics in the proportional hazards model. J. Multivariate Anal. 99, 777-786.

[19] Dykstra, R., Kochar, S. C. and Rojo, J. (1997). Stochastic comparisons of parallel systems of heterogeneous exponential components. J. Statist. Plann. Inference 65, 203-211.

[20] Fernández-Ponce, J. M., Kochar, S. C. and Muñoz-Perez, J. (1998). Partial orderings of distributions based on right-spread functions. Journal of Applied Probability 35, 221-228. 
[21] Furman, E. (2008). On a multivariate gamma distribution. Statistics \& Probability Letters 15, 2353-2360.

[22] Furman E. and Landsman, Z. (2006). Tail variance premium with applications for elliptical portfolio of risks. ASTIN Bulletin 36, 433-462.

[23] Genest, C., Kochar, S. and Xu, M. (2009). On the range of heterogeneous samples. Journal of Multivariate Analysis 100, 1587-1592.

[24] Hu T., Chen J. and Yao J. (2006) Preservation of the location independent risk order under convolution. Insurance: Mathematics \& Economics 38, 406412.

[25] Kaas, R., Goovaerts, M., Dhaene, J. and Denuit, M. (2001). Modern Actuarial Risk Theory. Kluwer Academic Publishers.

[26] Karlin, S. Total positivity. Vol. I, Stanford University Press, Stanford, CA, 1968.

[27] Khaledi, B. and Kochar, S. C. (2000). Some new results on stochastic comparisons of parallel systems. J. Appl. Probab. 37, 1123-1128.

[28] Khaledi, B. and Kochar, S. C. (2000). Sample range-some stochastic comparisons results. Calcutta Statistical Association Bulletin 50, 283-291.

[29] Khaledi, B. and Kochar, S. C. (2002). Stochastic Orderings among Order Statistics and Sample Spacings. Uncertainty and Optimality - probability, 
statistics and operations research. 167-203, edited by J.C. Misra. World Scientific Publications, Singapore.

[30] Khaledi, B.-E and Kochar, S. C. (2004). Ordering convolutions of gamma random variables. Sankhyā 66, 466-473.

[31] Khaledi, B.-E and Kochar, S. C. (2006). Weibull Distribution: Some Stochastic Comparisons Results. J. Statist.Plan. Inf. 136, 3121-3129.

[32] Kim, S.H. and David, H.A. (1990). On the dependence structure of order statistics and concomitants of order statistics. J. Statist. Plann. Inference 24, 363-368.

[33] Kochar, S. C. (1989). On extensions of DMRL and related partial orderings of life distributions. Communications in Statistics-Stochastic Models 5, 235245.

[34] Kochar, S. C. and Korwar, R. (1996). Stochastic orders for spacings of heterogeneous exponential random variables. J. Multivariate Anal. 59, 272-281.

[35] Kochar, S. C. and Ma, C. (1999). Dispersive ordering of convolutions of exponential random variables. Statistics \& Probability Letters 43, 321-324.

[36] Kochar, S. C. and Rojo, J. (1996). Some new results on stochastic comparisons of spacings from heterogeneous exponential distributions. J. Multivariate Anal. 57, 69-83. 
[37] Kochar, S., Li, X. and Shaked, M. (2002). The total time on test transform and the excess wealth stochastic order of distributions. Advances in Applied Probability 34, 826-845.

[38] Kochar, S. C. and Wiens, D. P. (1987). Partial orderings of life distributions with respect to their aging properties. Naval Research Logistics 34, 823-829.

[39] Kochar, S. and Xu, M. (2007). Stochastic comparisons of parallel systems when components have proportional hazard rates. Probability in the Engineering and Informational Science 21, 597-609.

[40] Kochar, S. and Xu, M. (2008). A new dependence ordering with applications. Journal of Multivariate Analysis 99, 2172-2184.

[41] Kochar, S. and Xu, M. (2009). Comparisons of parallel systems according to the convex transform order. Journal of Applied Probability, 46, 342-352.

[42] Kochar, S. and Xu, M. (2010). On the right spread order of convolutions of heterogeneous exponential random variables. Journal of Multivariate Analysis 101, 165-176.

[43] Korwar, R. M. (2002). On stochastic orders for sums of independent random variables, Journal of Multivatiate Analysis 80, 344-357.

[44] Joe, H. (1997). Multivariate models and dependence concepts. London: Chapman \& Hall. 
[45] Lehmann, E. L. (1966). Some concepts of dependence, Ann. Math. Statist. 37, 1137-1153.

[46] MacGillivray, H.L. and K.P. Blanda (1988). The relationships between skewness and kurtosis. Australian Journal of Statistics 30, 319-337.

[47] Marshall, A. W. and Olkin, I. (1979). Inequalities : Theory of Majorization and Its Applications. Academic Press, New York.

[48] Marshall, A. W. and Olkin, I. (2007). Life Distributions. Springer, New York.

[49] Mitrinović, D. S. (1970). Analytic Inequalities. Springer Verlag, Berlin.

[50] Müller, A. and Stoyan, D. (2002) Comparison methods for stochastic models and risks. John Wiley \& Sons, New York.

[51] Saunders, I. W. and Moran, P. A. (1978). On the quantiles of the gamma and F distributions. Journal of Applied Probability 15, 426-432.

[52] Nelsen, R. B. (2006) An Introduction to Copulas. Lecture Notes in Statistics. Springer, New York.

[53] Păltănea, E. (2008). On the comparison in hazard rate ordering of fail-safe systems. Journal of Statistical Planning and Inference 138, 1993-1997. 
[54] Pledger, P. and Proschan, F. (1971). Comparisons of order statistics and of spacings from heterogeneous distributions. Optimizing Methods in Statistics. Academic Press, New York., 89-113. ed. Rustagi, J. S.

[55] Proschan, F. and Sethuraman, J. (1976). Stochastic comparisons of order statistics from heterogeneous populations, with applications in reliability. $J$. Multivariate Anal. 6, 608-616.

[56] Sathe, Y. S. (1988). On the correlation coefficient between the first and the $\mathrm{r}$-th smallest order statistics based on $\mathrm{n}$ independent exponential random variables. Comm. Statist. Theory Meth. 17, 3295-3299.

[57] Scarsini, M. (1984). On measures of concordance. Stochastica 8, 201-218.

[58] Sen, P. K. (1970). A Note on Order Statistics for Heterogeneous Distributions. Annals of the Institute of Statistical Mathematics 41, 2137-2139.

[59] Shaked, M. and Shantikumar, J. G. (1998). Two variability orders. Probability in the Engineering and Informational Sciences 12, 1-23.

[60] Shaked, M. and Shanthikumar, J. G. (2007). Stochastic Orders and Their Applications. Springer, New York.

[61] Shanthikumar, J. G. and Yao, D. D. (1991). Bivariate Characterization of Some Stochastic Order Relations. Advances in Applied Probability 23 642-659. 
[62] Sordo, M. A. (2009). Comparing tail variabilities of risks by means of the excess wealth order. Insurance: Mathematics and Economics, 45, 466-469.

[63] Van Zwet, W. R. (1970). Convex transformations of random variables. Mathematical Centre Tracts No. 7, Second editinon. Mathematical Centre, Amsterdam.

[64] Xu, M. and Li, X. (2008). Some further results on the winner's rent in the second-price business auction. Sankhya 70, 124-133.

[65] Yu, Y. (2009). Stochastic ordering of exponential family distributions and their mixtures. Journal of Applied Probability 46, 244-254.

[66] Zhao, P., Li, X. and Balakrishnan, N. (2009). Likelihood ratio order of the second order statistic from independent heterogeneous exponential random variables. Journal of Multivariate Analysis 100, 952-962.

[67] Zhao, P., and Balakrishnan, N. (2009). Characterization of MRL order of fail-safe systems with heterogeneous exponential components. Journal of Statistical Planning and Inference, 139, 2920-2932.

[68] Zhao, P., and Balakrishnan, N. (2009). Mean residual life order of convolutions of heterogenous exponential random variables. Journal of Multivariate Analysis, 100, 1792-1801. 
[69] Zhao, P., and Li, X. (2009). Stochastic order of sample range from heterogeneous exponential random variables. Probability in the Engineering and Informational Sciences 23, 17-29. 